\title{
Cluster lenses
}

\section{Jean-Paul Kneib • Priyamvada Natarajan}

\begin{abstract}
Clusters of galaxies are the most recently assembled, massive, bound structures in the Universe. As predicted by General Relativity, given their masses, clusters strongly deform space-time in their vicinity. Clusters act as some of the most powerful gravitational lenses in the Universe. Light rays traversing through clusters from distant sources are hence deflected, and the resulting images of these distant objects therefore appear distorted and magnified. Lensing by clusters occurs in two regimes, each with unique observational signatures. The strong lensing regime is characterized by effects readily seen by eye, namely, the production of giant arcs, multiple images, and arclets. The weak lensing regime is characterized by small deformations in the shapes of background galaxies only detectable statistically. Cluster lenses have been exploited successfully to address several important current questions in cosmology: (i) the study of the lens(es)_understanding cluster mass distributions and issues pertaining to cluster formation and evolution, as well as constraining the nature of dark matter; (ii) the study of the lensed objects-probing the properties of the background lensed galaxy population-which is statistically at higher redshifts and of lower intrinsic luminosity thus enabling the probing of galaxy formation at the earliest times right up to the Dark Ages; and (iii) the study of the geometry of the Universe - as the strength of lensing depends on the ratios of angular diameter distances between the lens, source and observer, lens deflections are sensitive to the
\end{abstract}

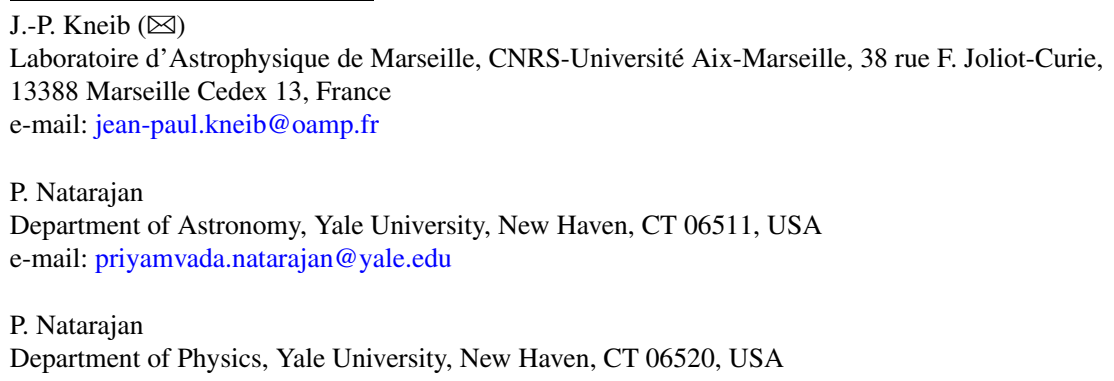


value of cosmological parameters and offer a powerful geometric tool to probe Dark Energy. In this review, we present the basics of cluster lensing and provide a current status report of the field.

Keywords Cosmology: observations · Galaxies: evolution · Galaxies: formation · Gravitational lensing

\section{Introduction and historical perspective}

In the early days of modern cosmology, soon after it was realized that the Universe was expanding (Hubble 1929, 1931; Lemaître 1931); Zwicky (1933) suggested that some unseen matter was the likely dominant mass component in clusters of galaxies. With remarkable prescience, Zwicky (1937) further noted that gravitational lensing by clusters would be an invaluable tool to: (i) trace and measure the amount of this unseen mass, now referred to as dark matter and currently thought to pervade the cosmos; and (ii) study magnified distant objects lying behind clusters. Zwicky's bold predictions were based on a profound and intuitive understanding of the properties of gravitational lensing. However, at that time, inadequate imaging technology coupled with the lack of theoretical understanding of structure formation in the Universe hampered further observational progress and discoveries of gravitational lensing effects.

Although the existence of clusters of galaxies has been recognized for nearly two centuries - they were first recognized by Messier and Herschel as "remarkable concentrations of nebulae on the sky" (see the review of Biviano 2000 and references therein) the study of clusters began in earnest only really in the 1950s. In particular, the publication of the first comprehensive cluster catalog of the nearby Universe by Abell in 1958, can be considered as a milestone that spurred the study of clusters of galaxies transforming it into an active observational research area.

In comparison, gravitational lensing theory developed much later in the 1960s with early theoretical studies demonstrating the usefulness of lensing for astronomy. In particular, Sjur Refsdal derived the basic equations of gravitational lens theory (Refsdal 1964a) and subsequently showed how the gravitational lensing phenomenon can be used to determine Hubble's constant by measuring the time delay between two lensed images (Refsdal 1964b). Following the discovery of quasars, Barnothy (1965) proposed gravitational lensing as a tool for the study of quasars. With the discovery of the first double quasar Q0957+561 by Walsh et al. (1979) gravitational lensing really emerged in astronomy as an active observational field of study.

The study of clusters of galaxies as astronomical objects on the other hand, came of age in the 1970s and early 1980s specially with the discovery of the X-ray emitting intra-cluster medium (Lea et al. 1973; Gull and Northover 1976; Bahcall and Sarazin 1977; Serlemistos et al. 1977; Cavaliere and Fusco-Femiano 1978) and the numerous studies of the stellar populations of galaxies in clusters (Bautz and Morgan 1970; Sandage 1976; Leir and van den Bergh 1977; Hoessl et al. 1980; Dressler 1980). However, there was no discussion of their lensing properties in theoretical papers till the 1980s. The paper by Narayan et al. (1984) is one of the earliest theoretical papers that explored in detail the possibility that clusters can act as powerful lenses. As an 
example, this paper explained large separation multiple quasars as likely "clusterassisted" lensing systems. Although such a possibility had been already proposed by Young et al. (1980), who discovered a cluster of galaxies near the first double quasar Q0957+561, it was not so obvious for most other systems.

The likely explanation for the lack of interest in cluster lensing research was probably the belief that clusters were rather diffuse/extended systems and therefore not dense enough to act as powerful light deflectors. Only with the establishment of the role of cold dark matter in structure formation, did it become clear that clusters are indeed repositories of vast amounts of dark matter that enable them to act as efficient lenses in the Universe. The theory of structure formation in the context of a cold dark matter dominated Universe was developed in a seminal paper by Blumenthal et al. (1984). An attractive feature of this cold dark matter hypothesis was its considerable predictive power: the post-recombination fluctuation spectrum was calculable, and it in turn governs the formation of galaxies and clusters. At that time, good agreement with the data was obtained for a Zel'dovich spectrum of primordial fluctuations. Several decades later, a version of this paradigm the $\Lambda$ Cold Dark Matter $(\Lambda \mathrm{CDM}$ hereafter) model which postulates the existence of a non-zero cosmological constant $\Omega_{\Lambda}$ is currently well established and is in remarkable agreement with a wide range of current observations on cluster and galaxy scales.

Nevertheless, it still came as quite a surprise when in 1986, Lynds and Petrosian (1989) and Soucail et al. (1987) independently discovered the first "giant arcs": the strongly elongated images of distant background galaxies in the core of massive clusters (see Fig. 1). This new phenomenon was then immediately identified by Paczynśki (1987) as the consequence of gravitational lensing by the dense centers of clusters, and was soon confirmed by the measurement of the redshift of the arc in Abell 370 (Soucail et al. 1988). The discovery of giant arcs revealed the existence of the strong lensing regime, however, as we know now, it only represents the tip of the iceberg!

Coupled with the growing theoretical understanding of the structure and assembly history of clusters, this observational discovery of cluster lensing opened up an entire new vista to probe the detailed distribution of dark matter in these systems. In 1990, Antony Tyson while obtaining deep CCD imaging of clusters, identified a "systematic alignment" of faint galaxies around cluster cores (Tyson et al. 1990). He then suggested that this weak alignment produced by the distortion due to lensing by clusters could be used to map dark matter at larger radii in clusters than strong lensing afforded. These two key discoveries of strong and weak lensing, respectively, opened up a rich, new field in astronomy, the study of "cluster lenses", which we discuss further in this review.

These observational discoveries stoked the theoretical community to produce a number of key papers in the first half of the 1990s that developed the theoretical framework for strong and weak lensing techniques. Several of the seminal papers date from this period, and theorists delved into quantifying this new territory of gravitational lensing. Some of the significant early papers are: Schneider (1984), Blandford and Narayan (1986), Blandford et al. (1989), Kochanek (1990), Miralda-Escudé (1991), Kaiser (1992), and Kaiser and Squires (1993). It is important to underline that significant advances in technology spurred the field dramatically during these years. The discovery of the lensing phenomenon in clusters was made possible thanks to 


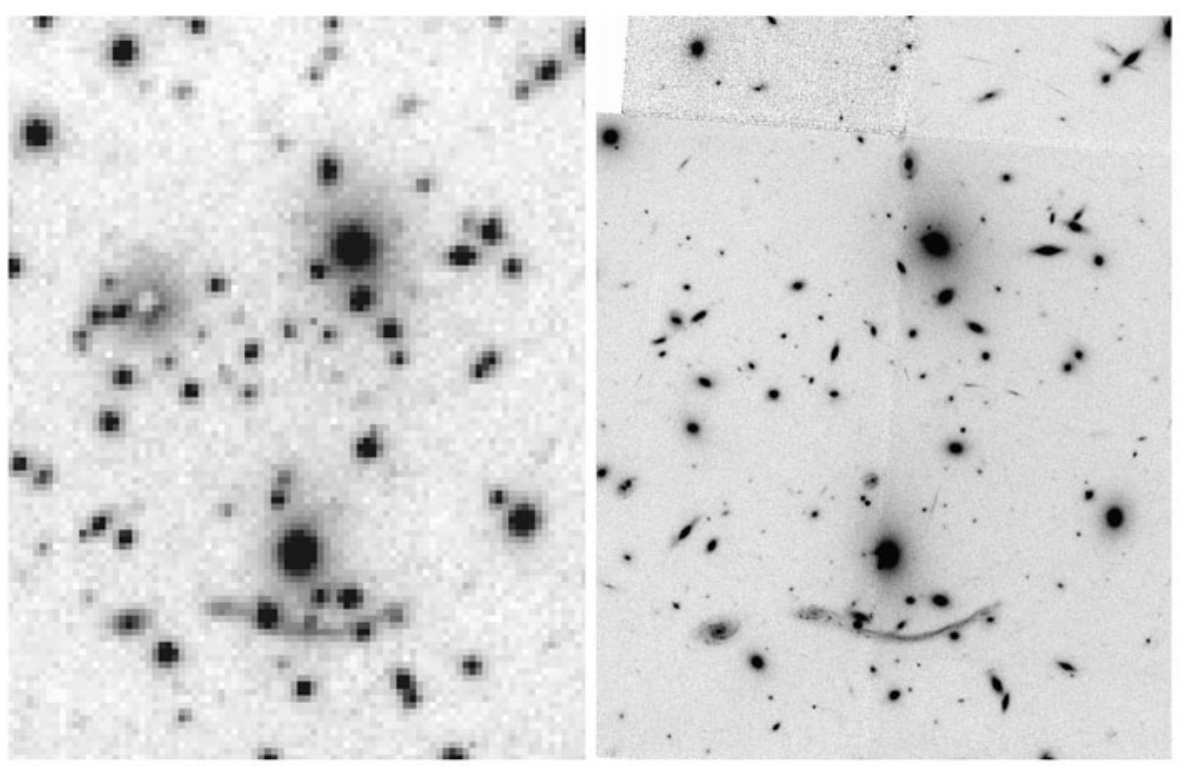

Fig. 1 The galaxy cluster Abell 370 as observed by CFHT in 1985 (left) with one of the first CCD cameras (R-band), in which the first gravitationally lensed arc was later identified (Lynds and Petrosian 1989; Soucail et al. 1987). For comparison, the image on the right shows the Hubble Space Telescope image of the same cluster Abell 370 taken with the WFPC-2 camera with the F675W filter in December 1995 (Soucail et al. 1999). Most of the bright galaxies seen are cluster members at $z=0.375$, whereas the arc, i.e. the highly elongated feature, is the image of a background galaxy at redshift $z=0.724$ (Soucail et al. 1988). The image is oriented such that North is on top, East to the left and the field of view is roughly $40 \times 60 \operatorname{arcsec}^{2}$

the successful development of CCD imaging that allowed deeper and sharper optical images of the sky, as well as deep spectroscopy - essential to measure the spectrum and the redshifts of the faint lensed background galaxies. Another technological revolution was in preparation at that time, a telescope above the atmosphere: the Hubble Space Telescope (HST). HST has dramatically impacted cluster lensing studies, and, in particular, that of the strong lensing regime. Although launched in 1991, HST did not make a strong impact at first, as its unforeseen "blurred vision" made the faint images of distant galaxies inadequate for lensing work. Nevertheless, even with the first HST-WFPC1 (Wide Field Planetary Camera) images of Abell 370 and AC114 one could already see the potential power of Hubble for lensing studies.

In December 1993, with the first successful servicing mission and the installation of the odd shaped WFPC2 camera, Hubble recovered its image sharpness, and it is not surprising that one of the first image releases following the installation of WFPC2 was the astonishing view of the cluster lens Abell 2218 (Kneib et al. 1996), which is iconic and has been included in most recent introductory astronomy textbooks.

Image sharpness is one of the key pre-requisites for studying lensing by clusters (e.g. Smail and Dickinson 1995) and unsurprisingly another requirement is a large image field of view. The strong lensing regime in clusters corresponds to the inner one arc-minute region around the cluster center. Typically, cluster virial radii are of the order of a few Mpc, which corresponds to $\sim 15$ arcminutes for a cluster at $z \sim 0.2$. 
Therefore, to go beyond the inner regions and to measure the weak lensing signal from cluster outskirts, cameras with a sufficiently large field of view are required to ideally cover the full size of a cluster in one shot (e.g. Kaiser et al. 1998; Joffre et al. 2000).

From the second half of the 1990s we have seen the rapid development of wide field imaging cameras such as: the UH8k followed by CFHT12k at CFHT (Canada France Hawaii Telescope); Suprime at the Subaru Telescope; WFI at the $2.2 \mathrm{~m}$ telescope at ESO (European Southern Observatory); the Megacam camera at CFHT; the Gigacam of Pan-STARRS (PS-1); the OmegaCam of the VST and soon the Dark Energy Camera at CTIO (Cerro Tololo Inter-American Observatory). These cameras are composed of a mosaic of many large format CCDs $(4 \mathrm{k} \times 2 \mathrm{k}$ or larger) allowing coverage of a large field (ranging from a quarter of a square degree up to a few square degrees). The making of these instruments was strongly motivated by the detection of the weak lensing distortion of faint galaxies produced by foreground clusters and intervening large-scale structure, the latter effect is commonly referred to as "cosmic shear".

In parallel, techniques to accurately measure the gravitational shear were also developed. The most well documented is the "KSB" technique (Kaiser et al. 1995) which is implemented in the commonly used IMCAT software package, ${ }^{1}$ which has been since improved by several groups. The accuracy of shape measurements for distorted background images is key to exploiting lensing effects. The difficulty in the shear measurement arises as galaxy ellipticities need to be measured extremely accurately given that there are other confounding sources that generate distortions. Spurious distortions are induced by the spatially and temporally variable PSF (Point Spread Function) as well as by intrinsic shape correlations that are unrelated to lensing (Crittenden et al. 2001, 2002). Corrections that carefully take into account these additional and variable sources of image distortion have been incorporated into shape measurement algorithms like LENSFIT ${ }^{2}$ (Miller et al. 2007; Kitching et al. 2008). Although the "KSB" technique has been quite popular due to its speed and efficiency, many new implementations for extracting the shear signal with the rapid increase in the speed and processing power of computers are currently available.

The first weak lensing measurements of clusters were reported with relatively small field of view cameras (Fahlman et al. 1994; Bonnet et al. 1994) but were soon extended to the larger field of view mosaic cameras (e.g. Dahle et al. 2002; Clowe and Schneider 2001, 2002; Bardeau et al. 2005, 2007). Two-dimensional dark matter mapping gets rapidly noisy as one extends over more than $\sim 2$ arcminutes from the cluster center due to a rapidly diminishing lensing signal. However, radial averaging of the shear field provides an effective way to probe the mass profile of clusters out to their virial radius and even beyond. This technique of inverting the measured shear profile to constrain the mass distribution of clusters is currently widely used. Combining constraints from the strong and weak lensing regime has enabled us to derive

\footnotetext{
${ }^{1}$ IMCAT software is available at http://www.ifa.hawaii.edu/ kaiser/imcat/.

${ }^{2}$ LENSFIT software is available at http://www.physics.ox.ac.uk/lensfit/.
} 
the dark matter density profile over a wide range of physical scales. As a consequence, gravitational lensing has become a powerful method to address fundamental questions pertinent to cluster growth and assembly.

Theoretically, as it is known that clusters are dominated by dark matter, enormous progress has been made in tracking their formation and evolution using large cosmological N-body simulations since the 1980s. Gravitational lensing is sensitive to the total mass of clusters, thereby enabling detailed comparison of the mass distribution and properties inferred observationally with simulated clusters. Lensing observations have therefore allowed important tests of the standard structure formation paradigm.

At the turn of the second millennium the new role of lensing clusters is its growing use as natural telescopes to study very high-redshift galaxies that formed during the infancy of the Universe (e.g. Franx et al. 1997; Pelló et al. 1999; Ellis et al. 2001). This became possible with deep spectroscopy on $4 \mathrm{~m}$ and then 8-10 m class telescopes that enable probing the high-redshift Universe, primarily by exploiting the lensing amplification and magnification ${ }^{3}$ produced by these natural telescopes (Pelló et al. 2001). Capitalizing on the achromatic nature of cluster lensing, various observatories functioning at different wavelengths of the electromagnetic spectrum have been deployed for these studies. In particular, the discovery and study of the population of sub-millimeter galaxies using SCUBA at the James Clerk Maxwell Telescope (JCMT hereafter; see the reviews by Blain et al. 2002; Smail et al. 2002; Kneib et al. 2004; Knudsen et al. 2005, 2008; Borys et al. 2005), the Caltech interferometer at Owens Valley (e.g. Frayer et al. 1998; Sheth et al. 2004), the IRAM interferometer (e.g. Neri et al. 2003; Kneib et al. 2005), the Very Large Array (VLA) (e.g. Smail et al. 2002; Ivison et al. 2002; Chapman et al. 2002) and Sub-Millimeter Array (SMA) (e.g. Knudsen et al. 2010) greatly benefited from the boost provided by the magnification effect of gravitational lensing in cluster fields. Similarly, observation of lensed galaxies in the mid-infrared with the ISOCAM mid-infrared camera on the Infra-red Space Observatory (ISO) satellite (Altieri et al. 1999; Metcalfe et al. 2003), followed with the Spitzer observatory (Egami et al. 2005) and now with the Herschel space observatory (Egami et al. 2010; Altieri et al. 2010) have pushed the limits of our knowledge of distant galaxies further. Gravitational lensing is now recognized as a powerful technique to count the faintest galaxies in their different classes: Extremely Red Objects (Smith et al. 2001); Lyman- $\alpha$ emitters at $z \sim 4-6$ (Hu et al. 2002; Santos et al. 2004; Stark et al. 2007); Lyman-break galaxies at $z \sim 6-10$ (Richard et al. 2008) as well as to study in detail the rare, extremely magnified individual sources (Pettini et al. 2000; Kneib et al. 2004; Egami et al. 2005; Smail et al. 2007, Swinbank et al. 2007, 2010) in the distant Universe.

Since March 2002, the installation of the new ACS camera onboard HST has provided further observational advances in the study and unprecedented use of cluster lenses (see Fig. 2). These are exemplified in the very deep and spectacular ACS images of Abell 1689 (Broadhurst et al. 2005; Halkola et al. 2006). This color image

\footnotetext{
${ }^{3}$ The magnification refers to the spatial stretching of the images by the gravitational lensing effect, however, the magnification cannot be recognized when the lensed object is not resolved by the observations (if the object is compact or if the PSF is broad) leading to an apparent amplification of the flux of the lensed object. In some cases, a lensed object can be tangentially magnified but radially amplified, the use of the terms magnification and amplification are thus sometimes mixed.
} 

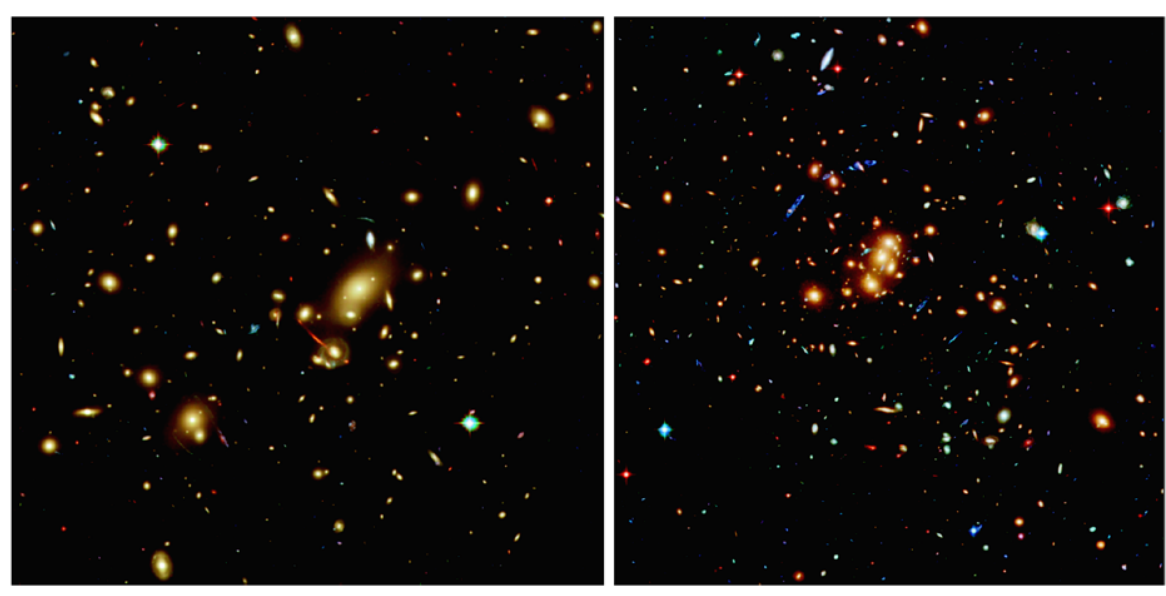

Fig. 2 Color image of two cluster lenses observed by HST-ACS: left panel Abell 2218 at $z=0.175$ and right panel $\mathrm{Cl} 0024+1654$ at $z=0.395$

reveals more than 40 multiple-image systems in the core of this cluster (Limousin et al. 2007) and well over a hundred lensed images in total. The dramatic increase in the number of strong lensing constraints that these observations provide in the cluster core has spurred important and significant new developments in mass reconstruction techniques (e.g. Diego et al. 2005a, 2005b; Jullo et al. 2007; Coe et al. 2008). With this amount of high quality data the construction of extremely high-resolution mass models of the cluster core are now possible. Mass models with high precision have enabled the use of this cluster to constrain the cosmological parameters $\Omega_{\mathrm{m}}$ and $\Omega_{\Lambda}$ (Link and Pierce 1998; Golse et al. 2004; Gilmore and Natarajan 2009; Jullo et al. 2010; D'Aloisio and Natarajan 2011a, 2011b). First observational constraints were attempted by Soucail et al. (2004), and more recent work by Jullo et al. (2010) has demonstrated the feasibility of this technique involving detailed modeling of deep ACS images coupled with comprehensive redshift determinations for the numerous multiple-image systems. Combining these cosmological constraints from the cluster lens Abell 1689 with those obtained from independent X-ray measurements and a flat Universe prior from WMAP, Jullo et al. (2010) find results that are competitive with the other more established methods like SuperNovae (Riess et al. 1998; Perlmutter et al. 1999) and Baryonic Acoustic Oscillations (Eisenstein et al. 2005). Therefore, in the very near future cluster strong lensing is likely to provide us with a viable complementary technique to constrain the geometry of the Universe and probe the equation of state of Dark Energy, which is a key unsolved problem in cosmology today.

This brief and non-exhaustive historical account of cluster lensing research summarizes some of the important scientific results gathered up to now and demonstrates the growing importance of cluster lensing in modern cosmology. This review is organized as follows: we first describe the key features of gravitational lensing in clusters of galaxies, starting with strong lensing, and then summarize the various weak lensing techniques as well as some recent developments in the intermediate lensing regime. 
We also dedicate a section to the lensing effect and measurements of galaxy halos in clusters which has provided new insights into the granularity of the dark matter distribution. The potency here arises from the ability to directly compare lensing inferred properties for substructure directly with results from high-resolution cosmological $\mathrm{N}$-body simulations. We then present the different uses of cluster lenses in modern cosmology. We start with the study of the lens: its mass distribution, and the relation of the lensing mass to other mass estimates for clusters. We then discuss the use of cluster lenses as natural telescopes to study faint and distant background galaxy populations. And lastly, we discuss the potential use of clusters to constrain cosmological parameters. Finally, we recap the important developments that are keenly awaited in the field, and describe some of the exciting science that will become possible in the next decade, focusing on future facilities and instruments. Cluster lensing is today a rapidly evolving and observationally driven field.

When necessary, we adopt a flat world model with a Hubble constant $H_{0}=$ $70 \mathrm{~km} \mathrm{~s}^{-1} \mathrm{Mpc}^{-1}$, a density parameter in matter $\Omega_{\mathrm{m}}=0.3$ and a cosmological constant $\Omega_{\lambda}=0.7$. Magnitudes are expressed in the AB system.

\section{Lensing theory as applied to clusters of galaxies}

\subsection{General description}

Clusters of galaxies are the largest and most massive bound structures in the Universe. Due to their large mass, galaxy clusters (as do galaxies) locally deform space-time (see Fig. 3). Therefore, the wave front of light emitted by a distant source traversing a foreground galaxy cluster will be distorted. This distortion occurs regardless of the wavelength of light as the effect is purely geometric. Moreover, for the most massive clusters the mass density in the inner regions is high enough to break the wave front coming from a distant source into several pieces, thereby occasionally producing multiple images of the same single background source. Background galaxies multiply imaged in this fashion tend to form the observed extraordinary gravitational giant arcs that characterize the so-called strong lensing domain. Strongly lensed distant galaxies will thus appear distorted and highly magnified. They are often referred to as arclets due to their noticeably elongated shape and preferential tangential alignment around the cluster center. Note, however, that their observed distorted shape is a combination of their intrinsic shape and the distortion induced by the lensing effect of the cluster.

When the alignment between the observer, a cluster and distant background galaxies is less perfect, then the distortion induced by the cluster will be less important and cannot be recognized clearly. Statistical methods are required to detect this change in shape of background galaxies seen in the weak regime. In the weak lensing regime, the observed shapes of background galaxies in the field of the cluster are typically dominated by their intrinsic ellipticities or even worse by the distortion of the imaging camera optics and the imaging point spread function (PSF) which is a function of position on the detector and may also vary with time. Thus, only a careful statistical analysis correcting the observed images for the various non-lensing induced distortion effects can reveal the true weak lensing signal. The shape changes induced in the outskirts of clusters in the weak regime are at the few percent level, while the strong lensing distortions are often larger, and are typically at the 10\%-20\% level. 


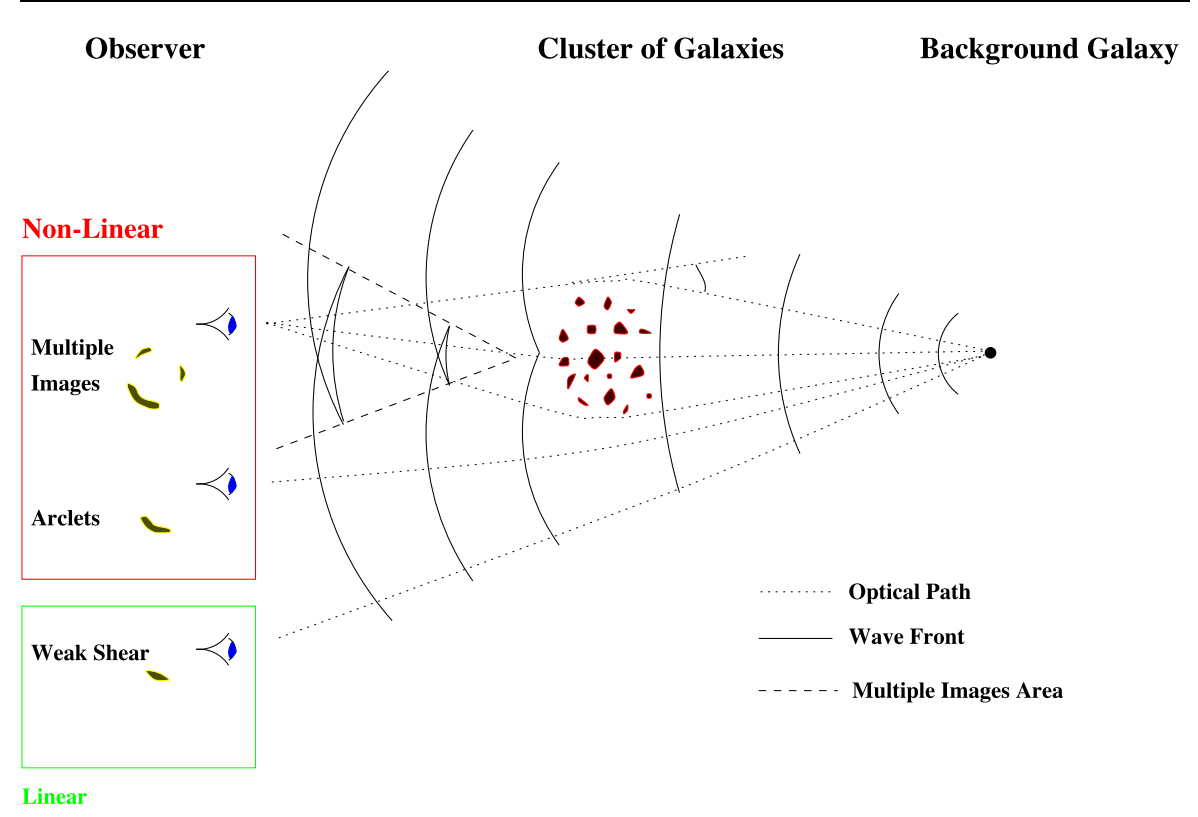

Fig. 3 Gravitational lensing in clusters: A simple schematic of how lensed images are produced delineating the various regimes: strong, intermediate and weak lensing (see text for a detailed description)

\subsection{Gravitational lens equation}

Before proceeding to the elegant mathematics of lensing, we first recap the assumptions needed to derive the basic lens equation. First, it is assumed that the "Cosmological Principle" (i.e. the Universe is homogeneous and isotropic) holds on large scales. The scales under consideration here are the ones relevant to the long-range gravitational force:

$$
L \sim \frac{c}{\sqrt{G \bar{\rho}}} \sim 2 \mathrm{Gpc},
$$

where $c$ is the speed of light, $G$ is the gravitational constant and $\bar{\rho}$ is the mean density of the Universe. The large-scale distribution of galaxies as determined by surveys like the 2 degree Field survey ( $2 \mathrm{dF}$ ), the Sloan Digital Sky Survey (SDSS) and the Cosmic Microwave Background (CMB) as revealed by the Cosmic Background Explorer (COBE), and the Wilkinson Anisotropy Probe (WMAP) satellites are in good agreement with the cosmological principle. The assumption of homogeneity and isotropy imposes strong symmetries on the metric that describes the Universe and allows solutions that correspond to both expansion and contraction. Symmetries restrict the metric that describes space-time to the following form:

$$
d s^{2}=c^{2} d t^{2}-a^{2}(t)\left(\frac{d r^{2}}{1-k r^{2}}+r^{2} d \theta^{2}+r^{2} \sin ^{2} \theta d \varphi^{2}\right),
$$

where $a(t)$ is the scale factor, and $k$ defines the curvature of the Universe. 
Fig. 4 A single deflector lensing configuration showing the relevant angles and distances that appear in the lens equation

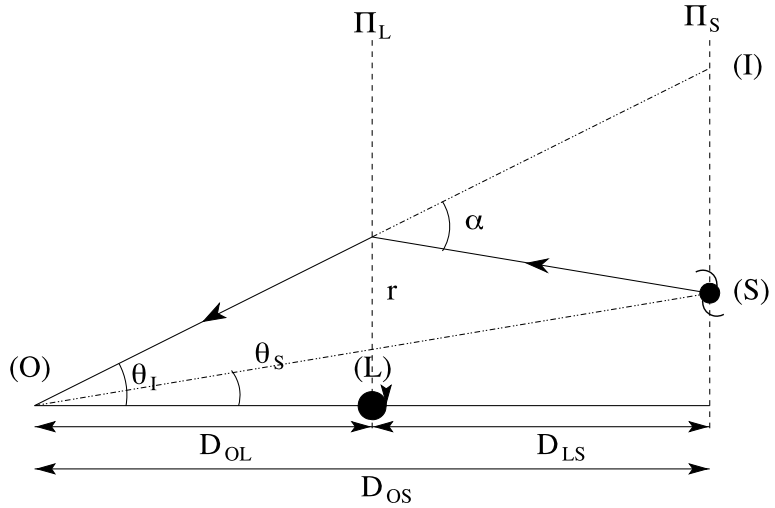

This metric will be locally perturbed by the presence of any dense mass concentration, such as individual stars, black holes, galaxies or clusters of galaxies. The Schwarzschild solution (e.g. Weinberg 1992) gives the form of the metric near a point mass, and is easy to generalize for a continuous mass distribution in the stationary weak field limit corresponding to $\Phi \ll c^{2}$ :

$$
d s^{2}=\left(1+\frac{2 \Phi}{c^{2}}\right) c^{2} d t^{2}-\left(1-\frac{2 \Phi}{c^{2}}\right) d r^{2},
$$

where $\Phi$ is the $3 \mathrm{D}$ gravitational potential of the mass distribution under consideration.

If we consider a simple configuration of a single thin deflecting lens (Fig. 4), the observer $(\mathrm{O})$ will see the image $(\mathrm{I})$ of the source $(\mathrm{S})$ deflected by the lens $(\mathrm{L})$. The geometric equation relating the position of the source $\vec{\theta}_{\mathrm{S}}$ to the position of the image

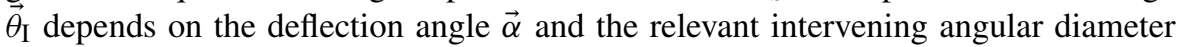
distances $D_{i j}$ in this case between the lens and source (denoted by $D_{\mathrm{LS}}$ ) and the observer and source (denoted by $D_{\mathrm{OS}}$ ):

$$
\vec{\theta}_{\mathrm{I}}=\vec{\theta}_{\mathrm{S}}+\frac{D_{\mathrm{LS}}}{D_{\mathrm{OS}}} \vec{\alpha}\left(\vec{\theta}_{\mathrm{I}}\right)
$$

The value of $\vec{\alpha}$ depends on the local perturbation of the mass on space-time measured at the location of $\vec{\theta}_{\mathrm{I}}$. The photon path follows a null geodesic that is defined by $d s^{2}=0$. Hence from (3), one can determine the travel time $t_{\mathrm{T}}$ for a given path length which in turn, is a function of the angle $\vec{\alpha}$. By applying Fermat's principle, which states that light follows the path with a stationary travel time, i.e. $d t_{\mathrm{T}} / d \vec{\theta}_{\mathrm{I}}=\overrightarrow{0}$, we can derive the value of the deflection $\vec{\alpha}$ as a function of the local Newtonian gravitational potential:

$$
\vec{\alpha}\left(\vec{\theta}_{\mathrm{I}}\right)=\frac{2}{c^{2}} \frac{D_{\mathrm{LS}}}{D_{\mathrm{OS}}} \vec{\nabla}_{\vec{\theta}_{\mathrm{I}}} \phi_{\mathrm{N}}^{2 \mathrm{D}}\left(\vec{\theta}_{\mathrm{I}}\right)
$$

where $\phi_{\mathrm{N}}^{2 \mathrm{D}}$ is the Newtonian gravitational potential projected in the lens plane.

Combining (4) and (5) we derive the lens equation under the thin lens approximation, which holds for a wide range of deflector masses, from stars to galaxies to 
Fig. 5 Lensing efficiency $\mathcal{E}=D_{\mathrm{LS}} / D_{\mathrm{OS}}$ for a given lens as a function of source redshift $z_{\mathrm{S}}$ for different cosmologies. The two sets of curves correspond to two different lens redshifts $z_{\mathrm{L}}=0.3$ and $z_{\mathrm{L}}=0.9$ and the solid lines correspond to $\Omega_{\mathrm{m}}=0.1, \Omega_{\Lambda}=0$; the dashed line to $\Omega_{\mathrm{m}}=1, \Omega_{\Lambda}=0$; and the dashed-dotted line to $\Omega_{\mathrm{m}}=0.1, \Omega_{\Lambda}=0.9$

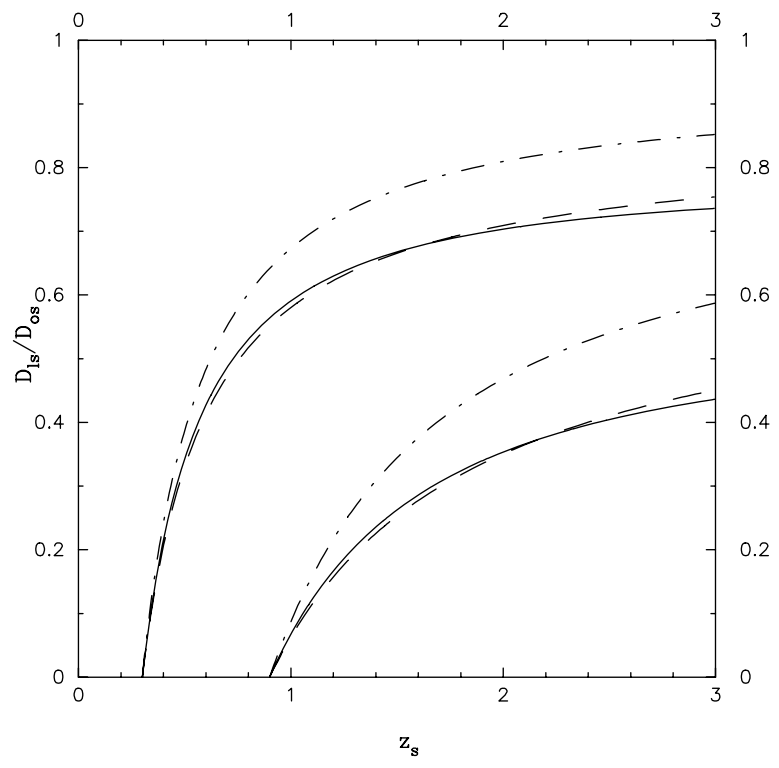

clusters of galaxies (see Schneider et al. 1992 for a more detailed derivation):

$$
\vec{\theta}_{\mathrm{S}}=\vec{\theta}_{\mathrm{I}}-\frac{2 \mathcal{E}}{c^{2}} \vec{\nabla} \phi_{\mathrm{N}}^{2 \mathrm{D}}\left(\vec{\theta}_{\mathrm{I}}\right)=\vec{\theta}_{\mathrm{I}}-\vec{\nabla} \varphi\left(\vec{\theta}_{\mathrm{I}}\right)
$$

The thin lens approximation is valid when the distances from the observer to the lens and source are significantly larger than the physical extent of the lens, an assumption that is strictly true for all galaxies and clusters. Above we define $\varphi$ as the lensing potential - a lensing normalized version of the Newtonian projected potential, and the distance ratio $\mathcal{E}=D_{\mathrm{LS}} / D_{\mathrm{OS}}$ which depends on the redshift of the cluster $z_{\mathrm{L}}$ and the background source $z_{\mathrm{S}}$, as well as—but only weakly-on the cosmological parameters $\Omega_{\mathrm{m}}$ and $\Omega_{\lambda}$. The distance ratio $\mathcal{E}$ measures the efficiency of a given lens at redshift $z_{\mathrm{L}}$. The factor $\mathcal{E}$ is an increasing function of the source redshift $z_{\mathrm{S}}$ (Fig. 5); therefore the larger the background source redshift, the stronger the deflection and distortion. This relation can be slightly more complex for sources located in the strong lensing regions. Note also that $\mathcal{E}$ is independent of the Hubble constant, therefore lensing deflection angles and deformations are independent of the value of $\mathrm{H}_{0}$.

It has also been shown that going beyond the thin lens approximation, the above lensing equation can be derived in the more general case (with (4) being the limiting case for Einstein-de Sitter space-time) by simply calculating the null geodesics intersecting an observer's world-line without partitioning light paths into near and far lens regions (see Pyne and Birkinshaw 1996 for a detailed derivation). The particularly interesting case is when more than one lensing deflector is responsible for producing the observed magnification and shear. Observations suggest that the lensing effect of most clusters are likely further amplified due to the existence of multiple additional mass concentrations aligned along the line of sight. Therefore, multiple lens planes 
will ultimately need to be taken into account for accurate mass modeling of cluster lenses. The precise coupling between the lensing effects of two adjacent masses depends on their transverse separation. Examining the two-screen gravitational lens, Kochanek and Apostolakis (1988) find, albeit for galaxy scale lenses, that their effects interact significantly for transverse separations less than $4 \times r_{0}$ where $r_{0}$ is the radius of the outer critical line of the singular potential. ${ }^{4}$ Independent lenses that are close in redshift almost always interact and these interactions can lead to either an increase or a decrease in the total cross section relative to the cross section of two isolated lenses depending on the system's geometry. The resultant image geometries in such cases are dominated by the effects of fold caustics. The deflection can be calculated for the two-screen lens configuration numerically and most current lens equation solvers are adapted to do so.

\subsection{Gravitational lens mapping}

The effect of gravitational lensing can be modeled as a mathematical transformation of source shapes into observed image shapes. The lensing transformation is thus a mapping from the source plane (S) to the image plane (I) [see Fig. 6]. In the case of a single lens plane, the Hessian of this transformation (also called the magnification matrix) relates to first order a source element of the image $\left(d \vec{\theta}_{\mathrm{I}}\right)$ to the source plane $\left(d \vec{\theta}_{\mathrm{S}}\right)$ in the following way, in Cartesian and polar coordinates, respectively:

$$
\frac{d \vec{\theta}_{\mathrm{S}}}{d \vec{\theta}_{\mathrm{I}}}=\mathcal{A}^{-1}=\left(\begin{array}{cc}
1-\partial_{x x} \varphi & -\partial_{x y} \varphi \\
-\partial_{x y} \varphi & 1-\partial_{y y} \varphi
\end{array}\right)=\left(\begin{array}{cc}
1-\partial_{r r} \varphi & -\partial_{r}\left(\frac{1}{r} \partial_{\theta} \varphi\right) \\
-\partial_{r}\left(\frac{1}{r} \partial_{\theta} \varphi\right) & 1-\frac{1}{r} \partial_{r} \varphi-\frac{1}{r^{2}} \partial_{\theta \theta} \varphi
\end{array}\right) .
$$

This matrix is referred to as the magnification/amplification matrix and it is conventionally written as

$$
\mathcal{A}^{-1}=\left(\begin{array}{cc}
1-\kappa-\gamma_{1} & -\gamma_{2} \\
-\gamma_{2} & 1-\kappa+\gamma_{1}
\end{array}\right),
$$

where the convergence is defined as $\kappa=\Delta \varphi / 2=\Sigma / \Sigma_{\text {crit }}$ and the shear vector (also often denoted as a complex number) $\vec{\gamma}=\left(\gamma_{1}, \gamma_{2}\right)$ as

$$
\gamma_{1}=\left(\partial_{x x} \varphi-\partial_{y y} \varphi\right) / 2 \quad \gamma_{2}=\partial_{x y} \varphi
$$

and the norm is given by

$$
2 \gamma=\sqrt{\left(\partial_{x x} \varphi-\partial_{y y} \varphi\right)^{2}+\left(2 \partial_{x y} \varphi\right)^{2}} .
$$

The term $\Sigma_{\text {crit }}$ is the critical lensing surface density defined as

$$
\Sigma_{\text {crit }}=\frac{c^{2}}{4 \pi G} \frac{D_{\mathrm{OS}}}{D_{\mathrm{LS}} D_{\mathrm{OL}}}=\frac{c H_{0}}{4 \pi G} \frac{D_{\mathrm{OS}}}{D_{\mathrm{LS}}} \frac{c / H_{0}}{D_{\mathrm{OL}}} .
$$

${ }^{4}$ Critical lines and caustics are defined in the next subsection. 


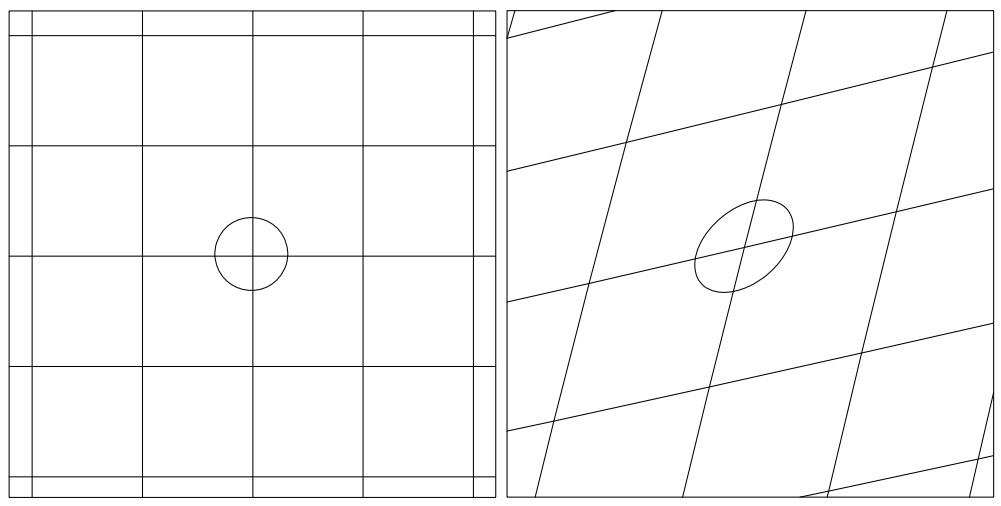

Fig. 6 Illustration of the effect of lensing: local deformation of a regular grid and a circle (left: source map) by a lens with constant value of the convergence $\kappa$ and the shear $\gamma$ over the region (right: image map)

It can be clearly seen that the critical surface mass density scales as

$$
\Sigma_{\text {crit }} \simeq 0.162\left(\frac{H_{0}}{70 \mathrm{~km} \mathrm{~s}^{-1} \mathrm{Mpc}^{-1}}\right)\left(\frac{D_{\mathrm{OS}}}{D_{\mathrm{LS}}}\right)\left(\frac{c / H_{0}}{D_{\mathrm{OL}}}\right) \mathrm{g} \mathrm{cm}^{-2} .
$$

For instance, given a cluster lens at $z_{\mathrm{L}}=0.3$ and a source at redshift $z_{\mathrm{S}}=1.0$, $\frac{D_{\mathrm{OS}}}{D_{\mathrm{LS}}}=1.567$ and $\frac{c / H_{0}}{D_{\mathrm{OL}}}=4.661$, yielding

$$
\Sigma_{\text {crit }} \simeq 1.18\left(\frac{H_{0}}{70 \mathrm{~km} \mathrm{~s}^{-1} \mathrm{Mpc}^{-1}}\right) \mathrm{g} \mathrm{cm}^{-2} .
$$

Thus, for a cluster with a depth of $\sim 300 \mathrm{kpc}$, the 3D mass density needed to reach the lensing critical surface mass density is about $10^{-24} \mathrm{~g} / \mathrm{cm}^{3}$, which corresponds to a density that is $\sim 10,000$ times the critical density of the Universe $\rho_{\text {crit }}$. Background galaxies viewed via a cluster region where the surface mass density is critical or higher are likely to be multiply imaged.

We can readily see that the magnification matrix is real and symmetric, therefore, it can be diagonalized, and can be written in its principal axes as follows:

$$
\mathcal{A}^{-1}=\left(\begin{array}{cc}
1-\kappa+\gamma & 0 \\
0 & 1-\kappa-\gamma
\end{array}\right)=(1-\kappa)\left[\left(\begin{array}{ll}
1 & 0 \\
0 & 1
\end{array}\right)+\frac{\gamma}{1-\kappa}\left(\begin{array}{cc}
1 & 0 \\
0 & -1
\end{array}\right)\right] \text {. }
$$

From this equation, we see that $1-\kappa$ describes the isotropic deformation, and the shear $\gamma$ describes the anisotropic deformation. Note that the quantity that is most directly measured from faint galaxy shapes is the reduced shear $g$ defined as $g=$ $\gamma /(1-\kappa)$.

The direction of the deformation (or equivalently of the shear) can be written as

$$
\tan 2 \theta_{\text {shear }}=\frac{2 \partial_{x y} \varphi}{\partial_{y y} \varphi-\partial_{x x} \varphi}
$$


As the direction of the shear is a ratio of the components of the lensing potential, the shear direction $\theta_{\text {shear }}$ will be independent (modulo 90 degrees) of the distance ratio $\mathcal{E}=D_{\mathrm{LS}} / D_{\mathrm{OS}}$ and thus will be independent of the source redshift $z_{\mathrm{S}}$. Only the intensity or magnitude of the shear will change with the source redshift $z_{\mathrm{S}}$.

\subsection{Critical and caustic lines}

The magnification $\mu$ is defined as the determinant of the magnification matrix and can be expressed as a function of $\kappa$ and $\gamma$ as

$$
\mu^{-1}=\operatorname{det}\left(\mathcal{A}^{-1}\right)=(1-\kappa)^{2}-\gamma^{2}=(1-\kappa)^{2}\left(1-g^{2}\right) .
$$

The magnification is infinite if one of the principal values of the magnification matrix is equal to zero, which implies that the reduced shear $g$ is equal to 1 or -1 . Thus, the locus in the image plane of infinite magnification defines two closed lines that do not intersect (as $g$ cannot be equal to 1 and -1 at the same location) and these are called the "critical lines". The corresponding lines in the source plane are called "caustic lines", they are also closed lines but contrary to the critical lines, they can intersect each other. In general, for a simple mass distribution, we can easily distinguish the two critical lines: the external critical line where the deformations are tangential, and the internal critical line where the deformations are radial. Note that these simple geometries for the critical and caustic lines do not hold strictly for more complex mass distributions (see Fig. 7 for examples of critical and caustic lines for different simple mass distribution).

For a circularly symmetric mass distribution, the equations for the critical lines are simple. The magnification matrix in polar coordinates simplifies to

$$
A^{-1}=\left(\begin{array}{cc}
1-\partial_{r r} \varphi & 0 \\
0 & 1-\frac{1}{r} \partial_{r} \varphi
\end{array}\right) .
$$

Thus both the critical and caustic lines (if they exist) are circles. In fact, substituting the equation of the tangential critical line: $r=\partial_{r} \varphi$ into the lensing equation to compute the caustic line, we find that the tangential caustic line is always restricted and reduces to a single point in the case of a circular mass distribution. It is also relatively easy to demonstrate that for a well behaved mass distribution the radial critical line is always located within the tangential critical line (Kneib 1993).

It is important to notice that for a circularly symmetric mass distribution, the projected mass enclosed within the radius $r$ can be written as

$$
M(r)=\frac{c^{2}}{4 G} \frac{D_{\mathrm{OS}} D_{\mathrm{OL}}}{D_{\mathrm{LS}}} r \partial_{r} \varphi(r)=\pi \Sigma_{\mathrm{crit}} r \partial_{r} \varphi(r) .
$$

At the tangential critical radius we have $r_{\mathrm{ct}}=\partial_{r} \varphi\left(r_{\mathrm{ct}}\right)$, thus the mass within the tangential critical radius (also referred to as the Einstein radius $r_{\mathrm{E}}$ ) is

$$
M\left(r_{\mathrm{E}}\right)=\pi \Sigma_{\text {crit }} r_{\mathrm{E}}^{2}
$$

The critical surface mass density $\Sigma_{\text {crit }}$ corresponds to the mean surface density enclosed within the Einstein radius. Thus the higher the mass concentration, the larger 


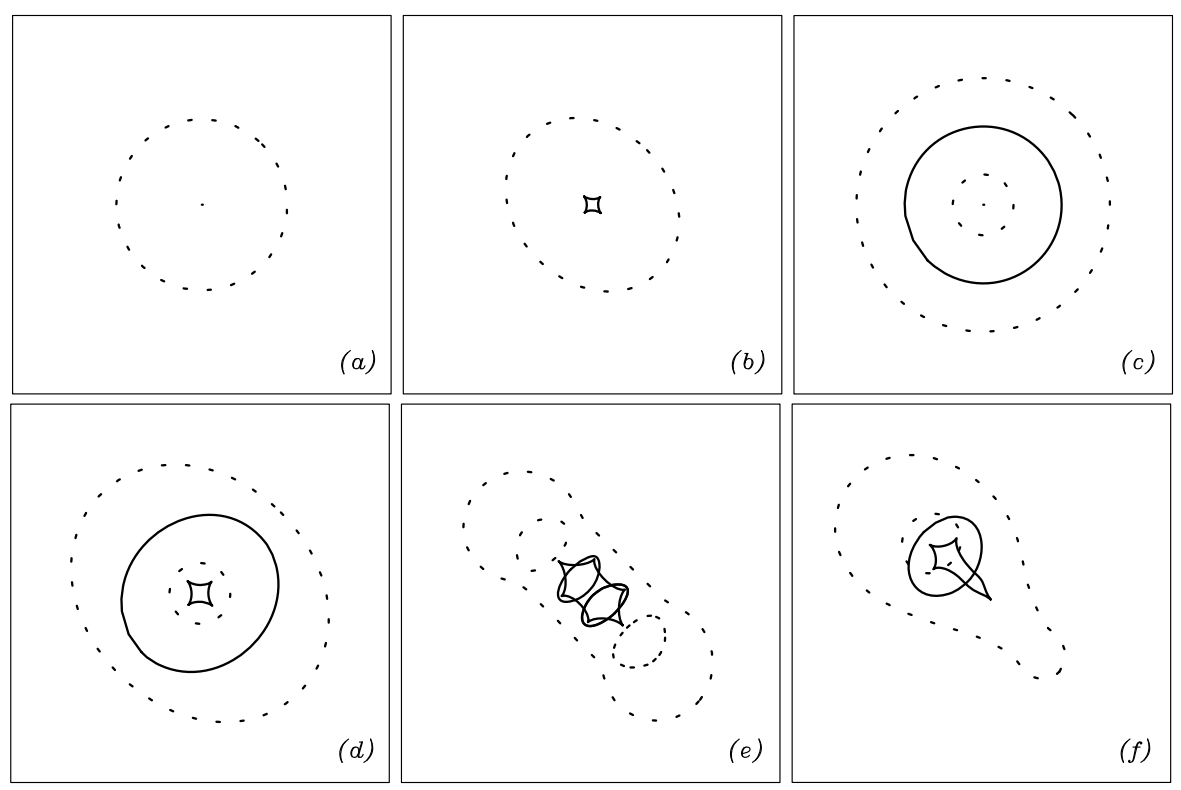

Fig. 7 Critical lines (dashed) and caustics (solid) for different classes of mass models: (a) for a singular isothermal circular mass distribution, the radial critical line is the central point, and the corresponding caustic line is at infinity, (b) a singular isothermal elliptical mass distribution, the tangential caustic line is an astroid, (c) a circular mass distribution with an inner slope shallower than isothermal mass distribution, in this case a radial critical curve appears, and both caustics are circles. (d) same as (c) but for an elliptical mass distribution, the relative size of both caustic lines will depend on the mass profile and the ellipticity of the mass distribution, (e) a bimodal mass distribution with two clumps of equal mass, similar to (d), and (f) for a bimodal distribution with unequal masses

the Einstein radius. For a given surface mass density profile, the size of the Einstein radius will depend on the redshift of the lens and the source as well as the underlying cosmology. The variation of $\Sigma_{\text {crit }}$ for a given source redshift as a function of the lens redshift shows that for a given lens mass distribution the most effective lens is placed at roughly less than half the source redshift.

Furthermore, the radial critical curve is defined as

$$
\partial_{r r} \varphi(r)=\partial_{r}\left(\frac{M(r)}{\pi \Sigma_{\text {crit }} r}\right)=1,
$$

thus, the position of the radial critical line depends on the gradient of the mass profile.

The above equations suggest that: (i) from the tangential critical curve location, the total mass enclosed within a circular aperture can be measured precisely, and (ii) from the radial critical curve, the slope of the mass profile near the cluster center can be strongly constrained. However, for an accurate estimate of the mass enclosed the redshifts of the cluster and the arc need to be known precisely. Furthermore, note that only the mass normalization scales directly with the value of $H_{0}$, but not the derived mass profile slope.

For the general non-circular case, the determination of the critical lines cannot be addressed analytically except for certain simple elliptical mass profiles (e.g. Kneib 


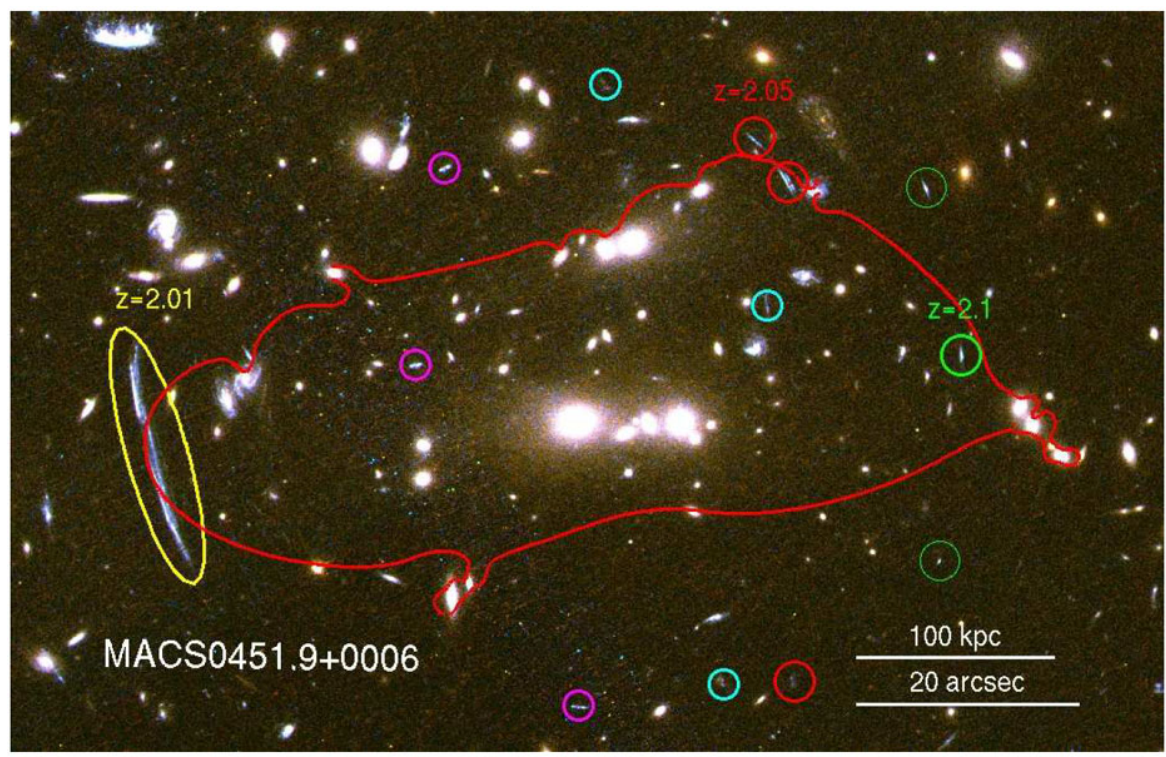

Fig. $8 H S T / A C S$ color image of MACSJ0451+00. The red curve shows the location of the critical line for a source at $z=2$. A giant arc at $z=2.01$, as well as different sets of multiple images are identified (each system of images is marked with a circle of the same color-the cyan and magenta identified multiple image have no spectroscopic redshift measurement yet) (Richard et al. private communication)

Fig. 9 Multi-scale marching square field splitting to map critical lines: the boxes represent the splitting squares and the red lines chart the critical curve contour. The imposed upper and lower limits for the box sizes are $10^{\prime \prime}$ (corresponding to the largest box shown) and $1^{\prime \prime}$, respectively. The $1^{\prime \prime}$ boxes are not plotted here for clarity. Figure adapted from Jullo et al. (2007) where more details may be found

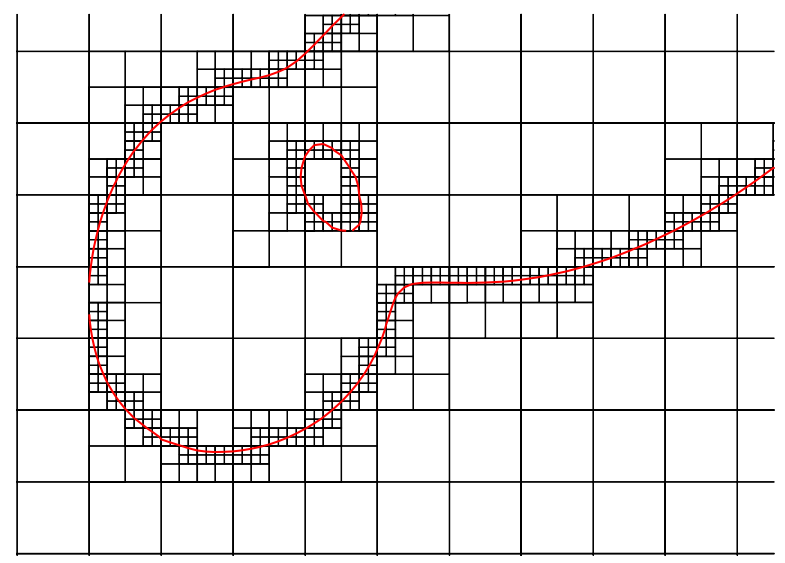

1993). The complexity of the shapes of critical lines can be seen for the lens model of MACS0451-02 (Fig. 8). Indeed, to solve for the critical line in complex lens mass models, one has to resort to numerical methods. Iterative methods are more economical in terms of CPU time. For example, Jullo et al. (2007) have implemented the "Marching Square" technique for computing critical lines (see illustration in Fig. 9).

The above property linking the total mass within the critical line to the area within the critical line does not hold exactly for the more general cases but it is still a good approximation if the mass distribution is not too different from the circularly sym- 
metric case (Kassiola and Kovner 1993). Hence identifying the characteristic sizes of the critical lines both radial and tangential in an observed cluster is the first important step toward measuring the mass and its degree of concentration in the inner regions.

\subsection{Multiple images}

\subsubsection{Definition}

Critical lines are virtual lines, and thus cannot be directly mapped. However, multiple images that straddle critical lines can easily be identified in high resolution images. For instance, tangentially distorted images are found near tangential critical lines and radially distorted ones near the radial critical lines. One often refers to tangential pairs or radial pairs, which are simple configurations that are easily recognizable (e.g. Miralda-Escudé and Fort 1993). For example, one can have triplets, quadruplets, quintuplets or even larger multiplicities of images of the same source depending on the complexity of the mass distribution.

The number of multiple images produced is simply the number of solutions of (6). It can be estimated easily using catastrophe theory (Thom 1989; Zeeman 1977; Erdl and Schneider 1993), according to which each time one crosses a caustic line in the source plane two additional lensed images are produced. For a non-singular mass distribution we expect to always have an odd number of multiple images (Burke 1981). However, some images are likely to be less magnified, or in fact, demagnified so that they are not observable, thereby complicating at first the task of counting the total number of multiple images produced. Often, the presence of a bright central galaxy in clusters scuppers the detection of the central demagnified image.

\subsubsection{Multiple-image symmetry}

Multiple images have different symmetries which can be summarized by the signs of the eigenvalues of the magnification matrix, we can thus in principle have three possibilities for the parities, which correspond to the symmetry of the source, denoted as $(+,+),(+,-)$ and $(-,-)$. For example we often talk about "mirror" symmetry, when we recognize a counter image as the flipped image of galaxy with a remarkably similar morphology. The image symmetry property is generally used to identify multiple images in what turns out to be a secure way, as we see in the pair configuration of Fig. 10.

Indeed, each time, one crosses a critical line (this corresponds to a change in sign of one of the eigenvalues of the magnification matrix), the parity of the image changes (Blandford and Narayan 1986; Schneider et al. 1992). For simple mass distributions, only three parities described above by the notation $(+,+),(-,-)$ and $(+,-)$ can be observed as shown in Fig. 11. Since for a simple mass distribution the radial critical line is always inside the tangential critical line, the parity $(-,+)$ is not physically allowed.

\subsubsection{Examples of multiple-image systems}

Massive clusters frequently produce multiple images, and this happens when the surface mass density of the cluster core is close to or larger than the critical surface mass 


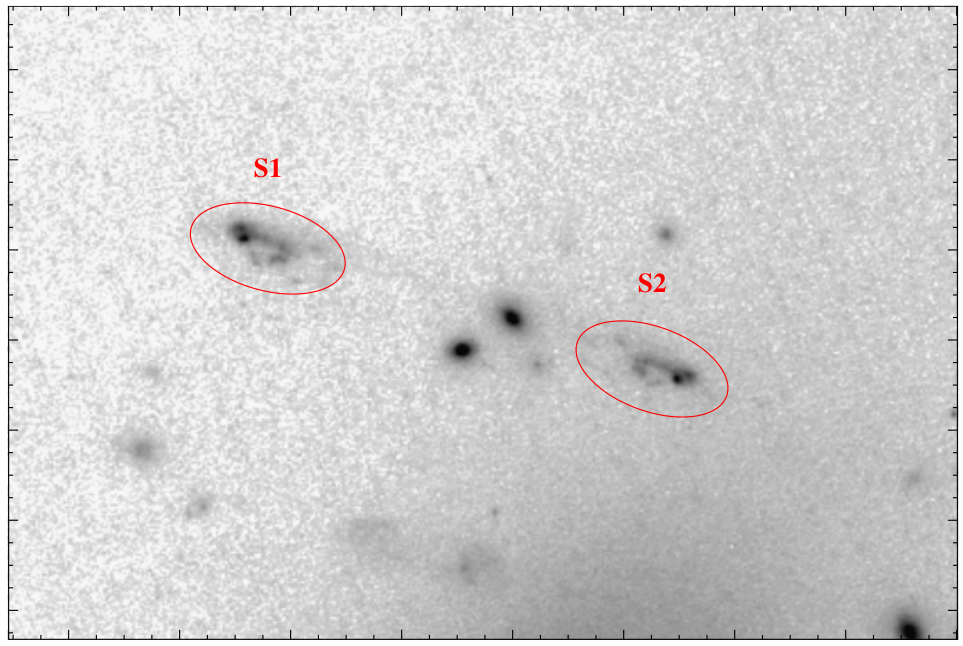

Fig. 10 The lensed pair S1-S2 in AC114. This galaxy at $z=1.867$ displays the surprising morphology of a hook, with an obvious change in parity (Smail et al. 1995; Campusano et al. 2001)

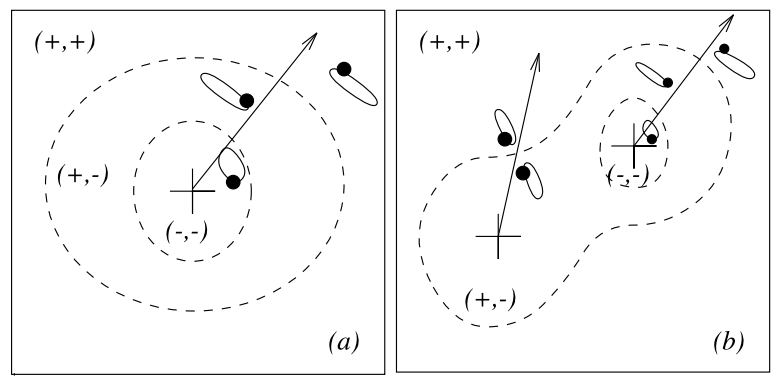

Fig. 11 Area in the image plane showing different image parities (indicated by the signs in parentheses): (a) in the case of a simple elliptical mass distribution, (b) in the case of a bimodal mass distribution. The dashed lines correspond to the critical lines. The arrow is just an indication of the radial direction of the closest mass clump. We note that while the deformations shown in this figure are completely arbitrary, the orientation of the images is portrayed accurately

density:

$$
\Sigma_{\text {crit }}=\frac{c^{2}}{4 \pi G} \frac{D_{\mathrm{OS}} D_{\mathrm{OL}}}{D_{\mathrm{LS}}},
$$

for given lens and source redshifts. The detailed configuration of multiple images can be used to unravel the structure of the mass distribution.

A cluster with one dominant clump of mass will produce (for the range of multipleimage configurations see Fig. 12) fold, cusp or radial arcs (e.g. MS2137.3-2353: Fort et al. 1992; Mellier et al. 1993; AC114: Natarajan et al. 1998; A383: Smith et al. 2001, 2003); a bimodal cluster can produce straight arcs (e.g. A2390: Pelló et al. 1991; C12236-04: Kneib et al. 1994), triplets (A370: Kneib et al. 1993; Bezecourt et al. 1999); a very complex structure with lots of massive halos in the core can produce multiple-image systems with seven or more images of the same source (e.g. 


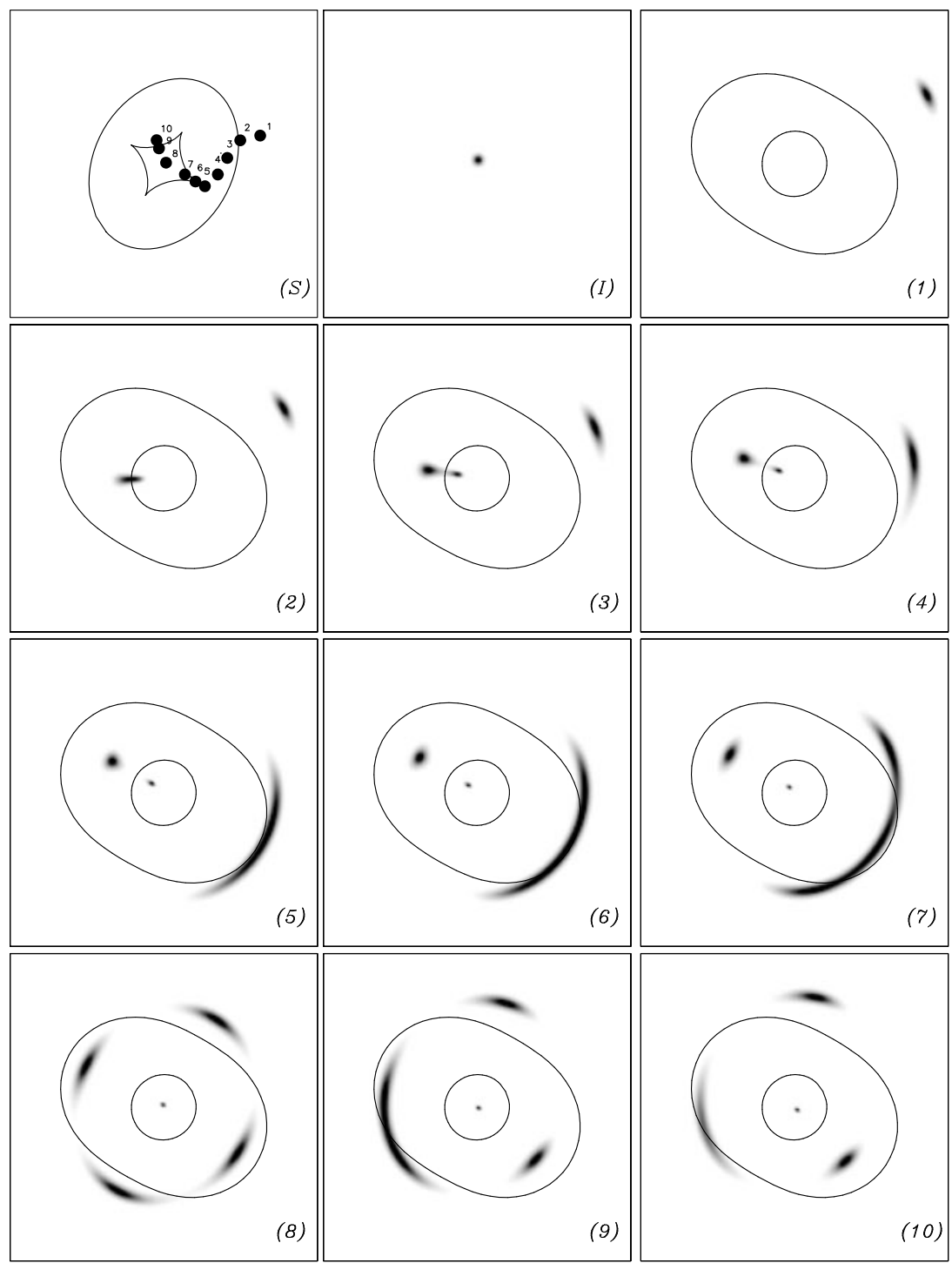

Fig. 12 Multiple-image configurations produced by a simple elliptical mass distribution. The panel (S) shows the caustic lines in the source plane and the positions numbered 1 to 10 denote the source position relative to the caustic lines. The panel (I) shows the image of the source without lensing. The panels (1) to (10) show the resulting lensed images for the various source positions. Certain configurations are very typical and are named as follows: (3) radial arc, (6) cusp arc, (8) Einstein cross, (10) fold arc

A2218, see Fig. 13). The presence of every nearby perturbing mass can typically add two extra images to a simple configuration if that mass is well positioned relative to the central core. Very elongated/elliptical clusters with appropriate inner density profile slopes can produce hyperbolic-umbilic catastrophes producing quintuple arc configurations such as the one seen in Abell 1703 (Limousin et al. 2008). A thorough 


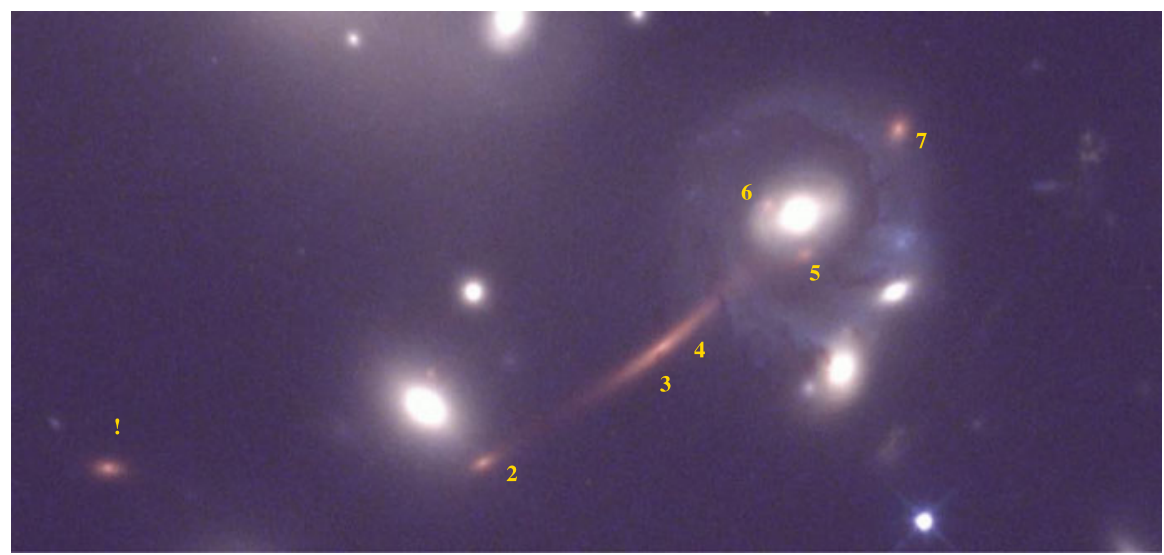

Fig. 13 A spectacular set of multiple images seen in the cluster Abell 2218 in the composite B, R, and I-band HST image. A distant E/S0 galaxy at $z=0.702$ is lensed into a 7-image configuration

description of exotic configurations has been discussed quite extensively in a paper by Orban de Xivry and Marshall (2009).

\subsubsection{Multiple-image identification}

Multiple images can be identified by their distinct properties. Traditionally, multiple images have been recognized as the images forming the giant arcs ( 3 images in the case of Abell 370, but only two images in the case of MS2137-17 or Cl2244-04). However, not every giant arc is composed of multiple images, for example it is most likely that the northern giant arc in Abell 963 is only a single image, and that the southern arc in Abell 963 is composed of two or three arclets (single images) from sources at different redshifts as revealed by their different colors. Multiple images can be recognized in terms of their (mirror) symmetry, which is of course best visible with high-resolution HST data. One of the classic examples is the "hook-pair" in AC114 (Fig. 10) where the image symmetry is readily identified. Furthermore, as lensing is achromatic, multiple images can be recognized by the similarity of their colors, or by their extreme brightness at a specific wavelength like in the sub-mm or in mid-infra red.

Finally, the secure way to identify and confirm the existence of a multiple-image system, is through the detailed modeling of the cluster lens itself. This allows one in principle to test if a set of images having similar morphology and colors can actually be multiple images of the same source. Calibrated lens models can predict the location of counter images and also predict the redshift of the multiply lensed source (Kneib et al. 1993, 1996).

Ultimately, for studying a large sample of massive clusters, one would likely need to develop automatic techniques to identify multiple-image systems based on their morphology, color and more sophisticated lens modeling software. Although such robotic processes are being developed (Sharon private communication), further developments are needed to make them completely user friendly. 

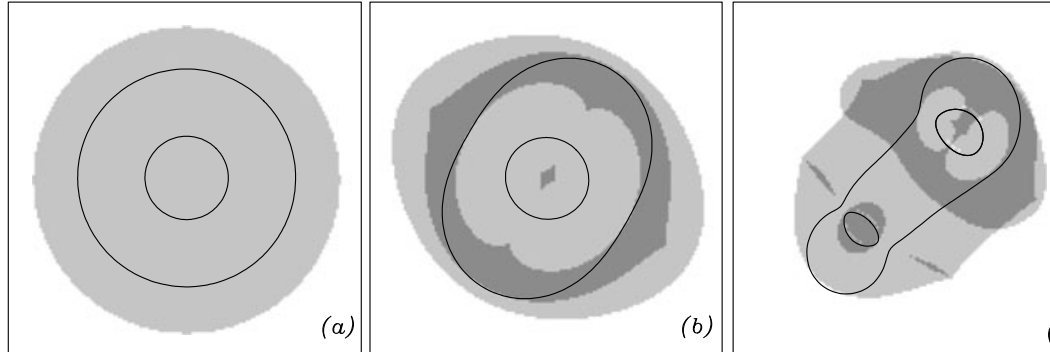

(c)

Fig. 14 Diagram showing the regions wherein multiple images are produced with the location of critical lines marked, for the case of different mass models (a) circular mass distribution; (b) an elliptical mass distribution and (c) a bimodal mass distribution. The grey scale areas indicate regions behind which we can expect a single background galaxy to be imaged into three (light grey) or five (dark grey) multiple images

\subsubsection{Multiple-image regions}

Multiple images are located in the central regions of clusters where the surface mass density is close to or higher than the lensing critical surface density. For a given source redshift one can compute the region conducive to multiple imaging and the expected multiplicity. This is easily computed, as for any given image position $\theta_{\mathrm{I}}$, we can determine the source position $\theta_{\mathrm{S}}$ using the lensing equation (4). Given a source position it is straightforward to determine whether $\theta_{\mathrm{S}}$ lies within a caustic curve or not. The expected number of images is given by $\left(1+2 N_{\mathrm{c}}\right)$ where $N_{\mathrm{c}}$ is the minimum number of caustic curves that need to be crossed to reach the position $\theta_{\mathrm{S}}$ (Fig. 14). The calculation of multiple-imaging regions can be useful to help observationally identify multiple-image counterparts (Fig. 15) particularly in the case of complicated mass distributions.

\subsection{First order shape deformations-shear}

Distant sources are only multiply imaged in the central regions of cluster where the surface mass density is sufficiently high. However, every observed galaxy image in the field of the cluster is deformed by lensing, typically in the weak regime. To first order, one can approximate the light distribution of a galaxy as an object with elliptical isophotes. In this event, the shape and size of galaxies can be defined in terms of the axis ratio and the area enclosed by a defined boundary isophote.

However, the real shapes of faint galaxies can be quite irregular and not well approximated by ellipses. We thus need to express the shape of a galaxy in terms of its pixelized surface brightness as measured on a digital detector. For this purpose, we use the moments of the light distribution to define shape parameters. If $I(\vec{\theta})$ is the surface brightness distribution of the galaxy under consideration, we can define the center of the image $\vec{\theta}_{\mathrm{c}}=\left(\theta_{i}^{\mathrm{C}}, \theta_{j}^{\mathrm{C}}\right)$ using the first moment of the $I(\vec{\theta})$ distribution:

$$
\vec{\theta}_{\mathrm{c}}=\frac{\int W(I(\vec{\theta})) \vec{\theta} d \vec{\theta}}{\int W(I(\vec{\theta})) d \vec{\theta}} .
$$




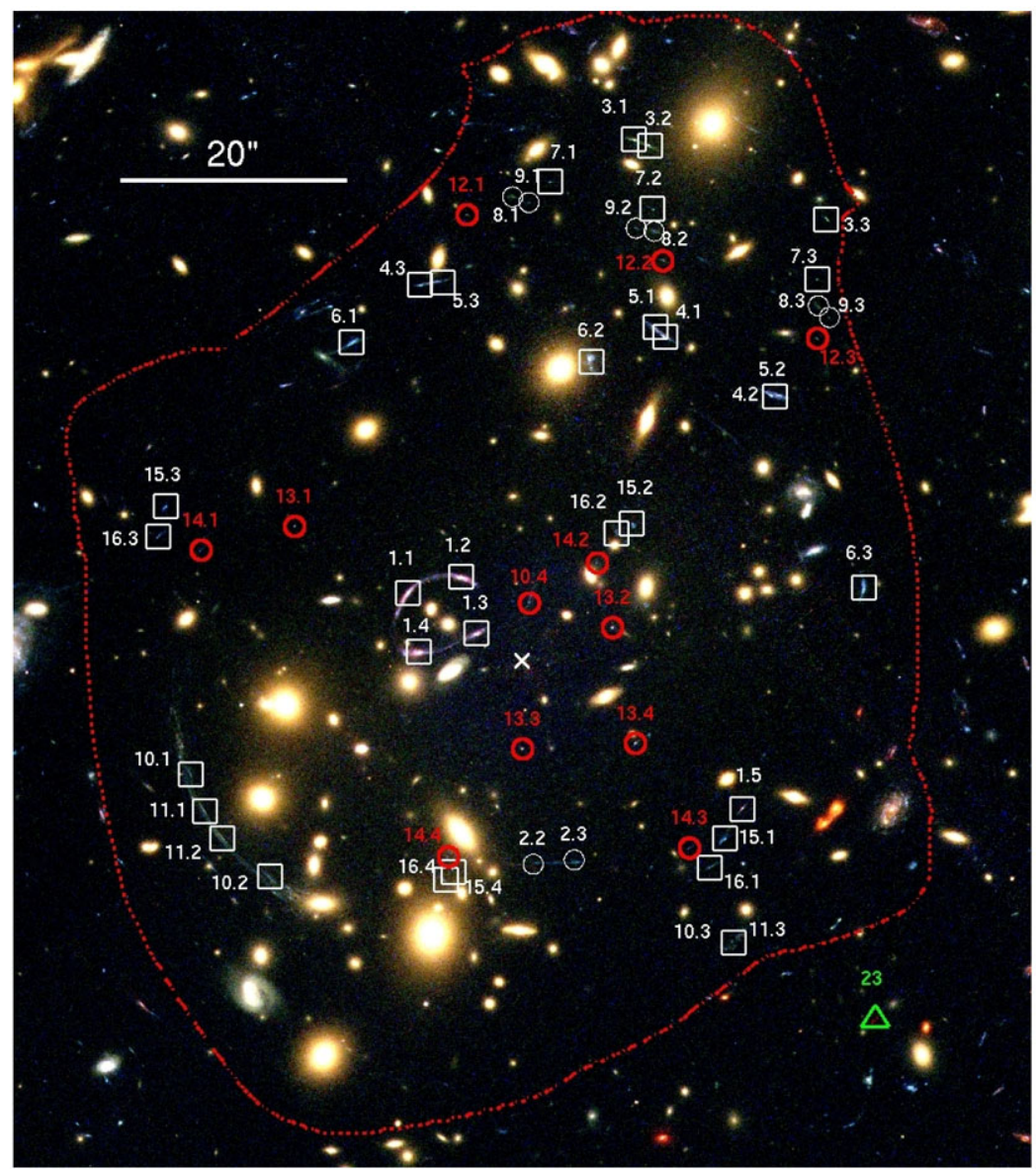

Fig. 15 Hubble ACS color image of Abell 1703 (image shown is from the combination of the F450W, F606W and F850LP filters), showing the location of all the multiply imaged systems. The white cross at the center of the image marks the location of the brightest cluster galaxy, which has been subtracted from this image for clarity. The red dashed line outlines the limit of the region where we expect multiple images from sources out to $z=6$ (figure from Richard et al. 2009)

Note that $W(I)$ is a weight/window function that allows the integrals above to be finite in the case of noisy data. The simplest choice for the function $W(I)$ is the Heaviside step function $H\left(I-I_{\text {iso }}\right)$ which is equal to 1 for $I(\vec{\theta})>I_{\text {iso }}$ where $I_{\text {iso }}$ is the isophote limiting the detection of the object, and 0 otherwise. The image center found is then taken as the center of the detection isophote. Another popular weight function that is frequently adopted is $W(I)=I \times H\left(I-I_{\text {iso }}\right)$, where the window function is now weighted by the light distribution within the isophote.

The second order moment matrix of the light distribution centered on $\vec{\theta}_{\mathrm{c}}$ :

$$
M_{i j}=\frac{\iint W(I(\vec{\theta}))\left(\theta_{i}-\theta_{i}^{\mathrm{C}}\right)\left(\theta_{j}-\theta_{j}^{\mathrm{C}}\right) d \theta_{i} d \theta_{j}}{\iint W(I(\vec{\theta})) d \theta_{i} \theta_{j}},
$$




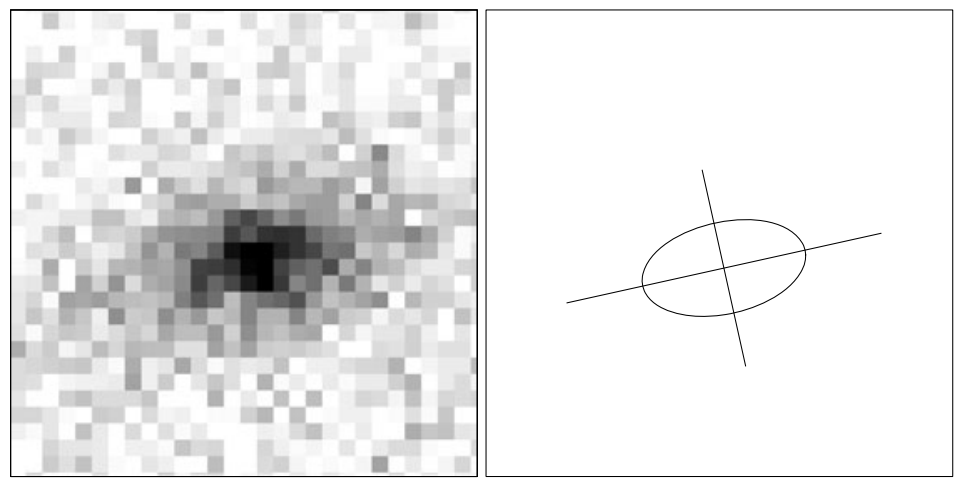

Fig. 16 A typical faint galaxy observed on a CCD image (left), and the equivalent ellipse defined from the second order moments (right)

allows us to define the size, the axis ratio and the orientation of the corresponding approximated ellipse. Indeed the moment matrix $M$ is positive definite and can be written in its principal axes as

$$
M_{i j}=R_{\theta}\left(\begin{array}{cc}
a^{2} & 0 \\
0 & b^{2}
\end{array}\right) R_{-\theta}
$$

where $a$ and $b$ are the semi-major and semi-minor axes, respectively, and $\theta$ the position angle of the equivalent ellipse, and $R_{\theta}$ is the rotation matrix of an angle $\theta$. Thus the moment matrix $M$ contains three parameters: the size of the galaxy, its ellipticity and its orientation (see illustration in Fig. 16).

It is useful to define a complex ellipticity which encodes both the shape parameter and the orientation of an observed galaxy.

There are, however, a number of ways to define the norm of the complex ellipticity, and the lensing community has experimented several notations:

$$
|\varepsilon|=\frac{a^{2}-b^{2}}{a^{2}+b^{2}} \quad|\delta|=\frac{a^{2}+b^{2}}{2 a b} \quad|\tau|=\frac{a^{2}-b^{2}}{2 a b} \quad|\epsilon|=\frac{a-b}{a+b} .
$$

With the complex ellipticity defined for example as

$$
\varepsilon=|\varepsilon| e^{2 i \theta} .
$$

The notation $\varepsilon$ was the first to be introduced, as it emerges naturally from the moment calculation, then $\tau$ and $\delta$ were introduced in the context of cluster lensing by Kneib (1993) and Natarajan and Kneib (1997). The advantage of this form is that the lens mapping can be written as a simple linear transformation from the image plane to the source plane which is mathematically convenient. Subsequently $\epsilon$ was adopted, and it has now become the standard definition, essentially because it is a direct estimator (modulo the PSF correction) of the measured quantity, which is the reduced shear $g$ as we show below. All ellipticity parameters are of course linked to each other, and in particular we have $\varepsilon=2 \epsilon /\left(1+|\epsilon|^{2}\right)$. 
With the various definitions in hand for the relevant parameters, we can now explicitly express the transformation produced by gravitational lensing on the shape of a background galaxy. First, it can be shown that the image of the center of the source corresponds to the center of the image in the case where the magnification matrix does not change significantly across the size of the image (Kochanek 1990; Miralda-Escudé 1991). This is generally adopted as the definition of the weak lensing regime as such a simplification does not hold in the strong lensing regime. To demonstrate this explicitly, one has to use the fact that the surface brightness is conserved by gravitational lensing as was first demonstrated by Etherington (1933), namely, $I\left(\vec{\theta}_{\mathrm{I}}\right)=I\left(\vec{\theta}_{\mathrm{S}}\right)$.

The lens mapping will transform the shape of the galaxy, by magnifying it and stretching it along the shear direction. This transformation can be written in terms of the moment matrix $M^{\mathrm{S}}$ (for the galaxy in the source plane) and $M^{\mathrm{I}}$ (for the galaxy in the image plane - that is, as observed) as follows:

$$
M^{\mathrm{S}}=A^{-1} M^{\mathrm{It}} A^{-1},
$$

or if the matrix $A^{-1}$ is not singular:

$$
M^{\mathrm{I}}=A M^{\mathrm{S} t} A .
$$

Note that ${ }^{\mathrm{t}} A$ is the transpose of matrix $A$.

These equations describe how the ellipse defining the source shape is mapped onto the equivalent ellipse of the image or vice versa. If we consider the size $\sigma=\pi a \times b$ of the equivalent ellipse, we can write

$$
\sigma_{\mathrm{S}}^{2}=\operatorname{det} M^{\mathrm{S}}=\operatorname{det} M^{\mathrm{I}} \cdot\left(\operatorname{det} A^{-1}\right)^{2}=\sigma_{\mathrm{I}}^{2} \cdot \mu^{-2} .
$$

Thus the overall size $\sigma_{\mathrm{S}}$ of the source is enlarged by the magnification factor $\mu$. Similarly, it is possible to write the lensing transformation for the complex ellipticity, which of course will depend on the ellipticity estimator chosen. For the ellipticity $\epsilon$, and using the complex notation, we have

$$
\epsilon_{\mathrm{S}}=\frac{\epsilon_{\mathrm{I}}-g}{1-g \epsilon_{\mathrm{I}}}, \quad \text { for }|g|<1,
$$

which corresponds to the region external to the critical lines, and

$$
\epsilon_{\mathrm{S}}=\frac{1-g^{*} \epsilon_{\mathrm{I}}^{*}}{\epsilon_{\mathrm{I}}^{*}-g^{*}}, \quad \text { for }|g|>1,
$$

which corresponds to the region inside the critical lines (the notation * denotes the transpose of a complex number).

In the weak regime, where the distortions are small $(|g| \ll 1)$ the lensing equation simplifies to

$$
\epsilon_{\mathrm{I}}=\epsilon_{\mathrm{S}}+g
$$


Thus the ellipticity of the image is just a linear sum of the intrinsic source ellipticity and the lensing distortion in this limit. Thus averaging the above equation over a number of sources yields the convenient fact that image ellipticities are a direct measure of the reduced shear $g$. Note, however, that these simplified equations mask observational limitations such as the PSF/seeing convolution and pixelization, all effects that contribute to and contaminate observed image shapes.

\subsection{Mass-sheet degeneracy}

The "mass sheet degeneracy" problem was recognized as soon as mass distributions began to be mapped using lensing observations (e.g. Falco et al. 1985) and the issue has been discussed in detail in Schneider et al. (1992), Schneider and Bartelmann (1997) and Bradač et al. (2004) in the context of weak lensing mass measurement. This degeneracy arises due to the lack of information needed to calibrate the total mass of clusters in the absence of a normalization scheme due to the simple fact that the addition of a constant surface mass density sheet leaves the measured shear unaltered.

Expressed mathematically, the magnification and shear are invariant under the following transformation:

$$
\kappa^{\prime}=(1-\lambda) \kappa+\lambda
$$

and

$$
\gamma^{\prime}=(1-\lambda) \gamma
$$

where $\lambda$ is the mass-sheet (denoting a sheet of constant surface mass density) added to the lens plane. Expressing the reduced shear with the above two equations we can show that

$$
g^{\prime}=g
$$

thus the reduced shear is conserved under this transformation. This means that for a given observed reduced shear field, one can only extract the surface mass density distribution $\kappa$ up to a constant factor given by the unknown value of $\lambda$.

There are several ways to break the mass sheet degeneracy, the obvious way is to use lensed sources from different source redshift planes. Indeed with, $\kappa\left(z_{1}\right)=$ $\mathcal{E}\left(z_{1}\right) / \mathcal{E}\left(z_{2}\right) \kappa\left(z_{2}\right)$, such a transformation is incompatible with the above invariance. Other methods to break the mass-sheet degeneracy, such as the inclusion of constraints from the strong lensing regime are discussed further in Sect. 3.4.3 on lens modeling.

\subsection{Higher order shape deformations-flexion}

The equations in the previous section assume that $\kappa$ and $\gamma$ and as a consequence the reduced shear $g$ are all constant across an image. This assumption fails when an image is physically large and/or when it is close to critical regions where the lensing distortion is changing rapidly. There are basically two effects that lensing produces on a background elliptical source: a shift in the peak flux at the center of the image compared to that of the fainter isophotes (while still preserving the surface 


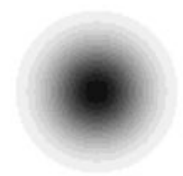

Unlensed

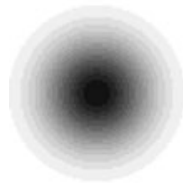

$\kappa$

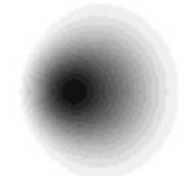

$\mathrm{F}_{1}$

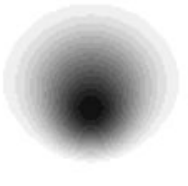

$\mathrm{F}_{2}$

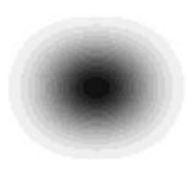

$\gamma_{1}$

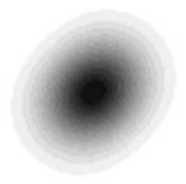

$\gamma_{2}$

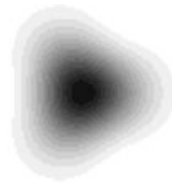

$\mathrm{G}_{1}$

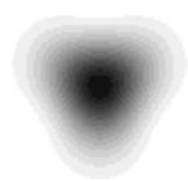

$\mathrm{G}_{2}$

Fig. 17 Decomposition of weak lensing distortions, illustrated for an unlensed Gaussian galaxy with a radius of 1 arcsec. The source has been distorted with $10 \%$ convergence/shear, and $0.28 \operatorname{arcsec}^{-1}$ flexion. The convergence $\kappa$, and 2 components of the first flexion $\left(F_{1}\right.$ and $\left.F_{2}\right)$; shear $\left(\gamma_{1}, \gamma_{2}\right)$; and second flexion $G_{1}$ and $G_{2}$ are shown. As mentioned, flexion causes the arciness or elongation of weakly lensed arcs once one combines $F, G$ and $\gamma$ (figure from Bacon et al. 2006)

brightness from the source to the image), and the distortion of the elliptical shape into an extended "banana" shape. Therefore, there is additional, valuable information that can be gleaned from higher order lensing effects.

To determine these higher order effects numerically, one needs to use higher orders of the lensing transformation using the Taylor expansion of the image shape. This was first investigated by Goldberg and Natarajan (2002), and followed up by Goldberg and Bacon (2005). A recent summary of the formalism and applications is reviewed in Bacon et al. (2005). Flexion is the significant third-order weak gravitational lensing effect responsible for the skewed and arc-like appearance of lensed galaxies. Flexion has two components: the first flexion, which is essentially the derivative of the shear field which contains local information about the gradient of the matter density (Goldberg and Natarajan 2002) and the second flexion which contains non-local information (Bacon et al. 2005 and Fig. 17). Flexion measurements can be used to measure density profiles and these reconstructions can be combined with those derived from the shear alone. One key advantage of using the flexion estimator is that it is not plagued by the mass sheet degeneracy as it is a higher order term, while its dispersion measure is comparable to that of the shear. Recent successful applications of flexion to map mass distributions can be found in Okura et al. (2008), Leonard et al. (2011) and Er et al. (2011).

Flexion arises from the fact that the shear and convergence are actually not constant within the image, and therefore to derive its explicit form we need to expand to second order:

$$
\theta_{i}^{\prime} \simeq A_{i j} \theta_{j}+\frac{1}{2} D_{i j k} \theta_{j} \theta_{k}
$$

with

$$
D_{i j k}=\partial_{k} A_{i j} .
$$


Using results from Kaiser (1995), we find that

$$
\begin{aligned}
& D_{i j 1}=\left(\begin{array}{cc}
-2 \gamma_{1,1}-\gamma_{2,2} & -\gamma_{2,1} \\
-\gamma_{2,1} & -\gamma_{2,2}
\end{array}\right), \\
& D_{i j 2}=\left(\begin{array}{lc}
-\gamma_{2,1} & -\gamma_{2,2} \\
-\gamma_{2,2} & 2 \gamma_{1,2}-\gamma_{2,1}
\end{array}\right) .
\end{aligned}
$$

By expanding the surface brightness as a Taylor series and using the relations above, we can approximate the lensed surface brightness of a galaxy in the weak lensing regime as

$$
f(\theta) \simeq\left\{1+\left[(A-I)_{i j} \theta_{j}+\frac{1}{2} D_{i j k} \theta_{j} \theta_{k}\right] \partial_{i}\right\} f^{\prime}(\theta) .
$$

This shows that the flexion can be written explicitly in terms of derivatives of the shear field. We define the flexion in terms of these shear derivatives, using the combination which is shown by Kaiser (1995) to directly give the gradient of the convergence:

$$
\begin{aligned}
\mathcal{F} & \equiv\left(\gamma_{1,1}+\gamma_{2,2}\right) i+\left(\gamma_{2,1}-\gamma_{1,2}\right) j \\
& =\nabla \kappa \\
& =|\mathcal{F}| e^{i \phi} .
\end{aligned}
$$

Since flexion is defined in terms of derivatives of the shear field, to measure it from observed shapes we need to be able to measure these derivatives, $\gamma_{i, j}$ with sufficient accuracy. This is becoming increasingly feasible with the availability of high quality imaging data. The first flexion probes the local density via the gradient of the shear field and quantifies the variation of the center of the different isophotal contours. The second flexion probes the non-local part of the gradient of the shear field and quantifies the shape variation and departure from elliptical symmetry.

Flexion has been incorporated as an additional constraint in the cluster mass reconstructions only recently as extremely high quality data is required to extract the flexion field and this is very challenging (see Leonard et al. 2011 for the case of Abell 1689). This higher order shape estimator, however, offers a powerful probe provided it can be measured accurately from observations (Leonard and King 2010; Er et al. 2011). As an illustration, we present the calculation of the flexion for the SIS model in Appendix A.4.

\section{Constraining cluster mass distributions}

In most cases, intermediate redshift $z \sim 0.2-0.5$ massive clusters are the most significant mass distribution along the line of sight, thus they can be represented by a single lens plane in concordance with the thin lens approximation. In the $\Lambda \mathrm{CDM}$ model, the probability of finding two massive clusters extremely well aligned along the line of 
sight (albeit separated in redshift) is extremely unlikely as clusters are very rare objects. Lensing deflections and distortions probe the two dimensional projected cluster mass along the line of sight. This allows us to constrain the two dimensional Newtonian potential, $\phi(x, y)$, resulting from the three-dimensional density distribution $\rho(x, y, z)$ projected onto the lens plane. The related projected surface mass density $\Sigma(x, y)$ is then given by

$$
4 \pi \mathrm{G} \Sigma(x, y)=\nabla^{2} \phi(x, y) .
$$

Often we are interested in the two-dimensional projected mass inside an aperture radius $R$ (particularly when comparing different mass estimators), which is defined explicitly as follows:

$$
M_{\text {aper }}(R)=2 \pi \int_{0}^{R} \Sigma(x) x d x,
$$

and the mean surface density inside the radius $R$ is given by

$$
\bar{\Sigma}(R)=\frac{1}{\pi R^{2}} \int_{0}^{R} 2 \pi x \Sigma(x) d x .
$$

The important quantities for lensing in clusters are primarily the deflection angle $\vec{\alpha}$ between the image and the source, the convergence $\kappa$, and the shear $\gamma$, which can all be conveniently expressed in terms of the projected potential:

$$
\left\{\begin{array}{l}
\vec{\alpha}(\vec{\theta})=\vec{\nabla}_{\vec{\theta}} \varphi(\vec{\theta}), \\
\kappa(\vec{\theta})=\frac{1}{2}\left(\frac{\partial^{2} \varphi}{\partial \theta_{1}^{2}}+\frac{\partial^{2} \varphi}{\partial \theta_{2}^{2}}\right) \\
\gamma^{2}(\vec{\theta})=\|\vec{\gamma}(\vec{\theta})\|^{2}=\frac{1}{4}\left(\frac{\partial^{2} \varphi}{\partial \theta_{1}^{2}}-\frac{\partial^{2} \varphi}{\partial \theta_{2}^{2}}\right)^{2}+\left(\frac{\partial^{2} \varphi}{\partial \theta_{1} \partial \theta_{2}}\right)^{2}
\end{array}\right.
$$

For a radially symmetric mass distribution, these expressions can be written as

$$
\left\{\begin{array}{l}
\kappa(x)=\frac{\Sigma(x)}{\Sigma_{\text {crit }}}, \\
\gamma(x)=\frac{\bar{\Sigma}(x)-\Sigma(x)}{\Sigma_{\text {crit }}}, \\
\vec{\alpha}(x)=\theta \frac{\bar{\Sigma}(x)}{\Sigma_{\text {crit }}}=\theta(\kappa(x)+\gamma(x))
\end{array}\right.
$$

where $x=D_{\mathrm{OL}} \theta$ is the radial physical distance. From this equation, we note that one can derive $\gamma$ directly from $\alpha$ and $\kappa$. This formulation is particularly useful when 
trying to compute an analytic expression of the lensing produced by a given mass profile.

\subsection{Strong lensing modeling}

\subsubsection{Modeling approaches}

Traditionally, modeling of the cluster mass distribution in the strong lensing regime is done using "parametric models" (e.g. Kneib et al. 1996; Natarajan and Kneib 1997). In these schemes the mass distribution is described by a finite number of mass clumps; some small scale (galaxy components) and some large scale (to represent the dark matter, X-ray gas in the Intra-Cluster Medium), each of which are described in turn by a finite number of parameters contingent upon the choice of mass profile deployed. The simplest mass distribution that is commonly employed is the circular Singular Isothermal Sphere (SIS), which is described by three parameters. The parameters are the position of its center $(x, y)$ and the value of the velocity dispersion $\sigma$, which in this case is a constant. Other mass distributions such as the PIEMD (Pseudo Isothermal Elliptical Mass Distribution) or NFW (Navarro-Frenk-White) profiles are often used in lensing analysis and their relevant parameters are described in the Appendix (see Sects. A.1-A.3).

Using a simple mass model makes sense when there are not many available observational constraints - indeed one needs to balance the number of model parameters to the number of observational constraints available in order to compute a sensible best-fit model. However, with recent deep images of cluster cores from HST a very large number of multiple images can readily be identified. For instance, more than 40 multiple-image systems have been identified in the massive cluster Abell 1689 by Limousin et al. (2007). The discovery and identification of such a large number of multiple images has dramatically increased the number of constraints available for mass modeling of massive clusters in the last decade. With the availability of a larger number of multiply imaged systems with redshift measurements, more accurate mass models (Fig. 18) are now possible.

Therefore, the number of allowable parameters required to describe the mass distribution of a cluster has also increased, leading obviously to a more accurate description of the mass profile in the cluster core. This is for example evident upon comparing the model of Kneib et al. (1993) with that of Richard et al. (2010) for the cluster Abell 370. In the case of "parametric models", the increase in the number of constraints translates to the fact that cluster mass distributions can now be described by a larger number of mass clumps and each of these clumps can be more complex (e.g. having elliptical mass distributions rather than circular, and a radial profile described by more parameters).

The increase in the number of available constraints has also lead to the development of new "non-parametric" methods, where no (or few) external priors are required to describe the mass distribution of clusters (Diego et al. 2005a; Saha and Williams 1997; Coe et al. 2010). Generically, in most of these "non-parametric" methods, the mass distribution is typically tessellated into a regular grid of smaller mass elements. Further details of such methods are discussed later on in this section. 


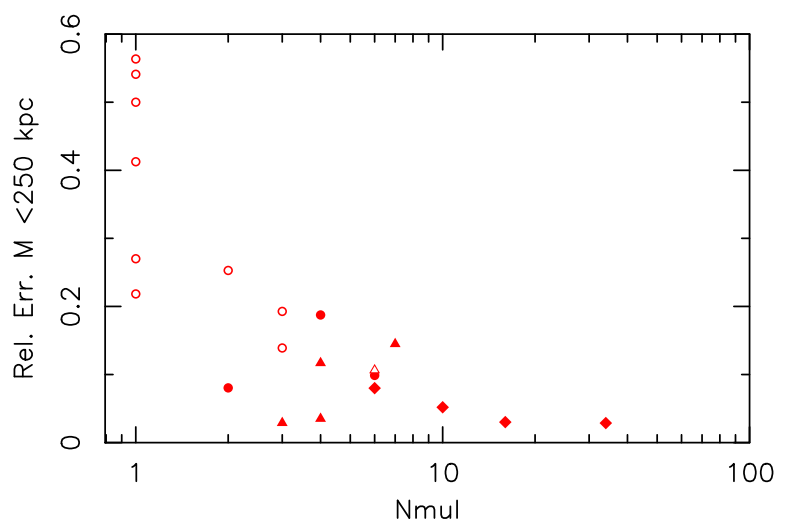

Fig. 18 Relative aperture mass error as a function of the number of multiple images as measured in Richard et al. (2010). Open symbols: clusters observed with WFPC2. Filled symbols: clusters observed with ACS. Symbols reflect the number of filters used to image the cluster (circle: 1 filter, triangle: 2 filters, diamond: 3 or more filters). One can see that with multi-band ACS data we can uncover more than 10 multiple-image systems for the most massive clusters, and thus achieve mass accuracy within $10 \%$ or so in cluster cores

\subsubsection{From simple to more complex mass determinations}

A particularly useful and popular mass estimate in the strong lensing regime is the mass enclosed within the Einstein radius $\theta_{\mathrm{E}}$, given by

$$
M\left(<\theta_{\mathrm{E}}\right)=\pi \Sigma_{\text {crit }} D_{\mathrm{OL}}^{2} \theta_{\mathrm{E}}^{2},
$$

where $\theta_{\mathrm{E}}$ is the location of the tangential critical line for a circular mass distribution, usually approximated by the tangential arc radius $\theta_{\text {arc }}$. It is a very handy expression that is independent of the mass profile for circularly symmetric cases. However, caution needs to be exercised when using this expression as often the arc used to derive the mass has an unknown redshift (thus $\Sigma_{\text {crit }}$ is not well defined), or the arc is a single image and therefore does not trace the Einstein radius or the mass distribution is very complex with a lot of substructure. Note that, however, for a singular isothermal sphere model, a single image cannot be closer than twice the Einstein radius since it will then have a counter image. In conclusion, this estimator tends to typically overestimate the mass in instances where the tangential arc is not multiply imaged or its redshift is unknown.

The radial critical line can be constrained when a radial arc is observed in the cluster core, this has now been done for a number of cluster lenses (e.g. Fort et al. 1992; Smith et al. 2001; Sand et al. 2002; Gavazzi et al. 2003). These features are important as they lie very close to the cluster core, and thus provide a unique probe of the surface mass density in the very center. Baryons are highly concentrated in the inner regions of clusters and they are expected to play an important role in possibly modifying the dark matter distribution on the smallest scales. The scales on which these effects are expected are accessible effectively with lensing data. Radial arcs have been used to probe the dark matter slope in the inner most regions of clusters 
(Sand et al. 2005, 2008; Newman et al. 2011), the results of these studies will be discussed in detail later on in this review.

The proper way to accurately constrain the mass in cluster cores is thus to use multiple images with preferably measured spectroscopic redshifts to absolutely calibrate the mass. To do this, one generally defines a likelihood $L$ for the observed data $D$ and parameters $\mathbf{p}$ of the model:

$$
\mathcal{L}=\operatorname{Pr}(D \mid \mathbf{p})=\prod_{i=1}^{N} \frac{1}{\prod_{j=1}^{n_{i}} \sigma_{i j} \sqrt{2 \pi}} \exp ^{-\frac{\chi_{i}^{2}}{2}},
$$

where $N$ is the number of systems, and $n_{i}$ the number of multiple images for the system $i$. The contribution from the multiple-image system $i$ to the overall $\chi^{2}$ can be simply given by

$$
\chi_{i}^{2}=\sum_{j=1}^{n_{i}} \frac{\left[\theta_{\mathrm{obs}}^{j}-\theta^{j}(\mathbf{p})\right]^{2}}{\sigma_{i j}^{2}}
$$

where $\theta^{j}(\mathbf{p})$ is the position of image $j$ predicted by the current model, whose parameters are $\mathbf{p}$ and where $\sigma_{i j}$ is the error on the position of image $j$.

The accurate determination of $\sigma_{i j}$ depends on the signal-to-noise of the image $\mathrm{S} / \mathrm{N}$ ratio. For extended images, a pixellated approach is the only accurate way to take the $\mathrm{S} / \mathrm{N}$ ratio of each pixel into account (Dye and Warren 2005; Suyu et al. 2006) but this is not optimal for cluster lenses with a large number of multiple-image systems. However, to a first approximation, the positional error of images can be determined by fitting a 2D Gaussian profile to the image surface brightness, which assumes that the background galaxy is compact and its surface brightness profile is smooth enough so that the brightest point in the source plane can be reliably matched to the brightest point in the image plane.

A major issue in the $\chi^{2}$ computation is how to match the predicted and observed images one by one. In models producing different configurations of multiple images (e.g. a radial system instead of a tangential system), the $\chi^{2}$ computation will fail and the corresponding model will then be rejected. This usually happens when the model is not yet well determined, and this can slow down the convergence of the modeling significantly. To get around this complexity, one often computes the $\chi^{2}$ in the source plane (by computing the difference in the source position for a given parameter sample p) instead of doing so in the image plane. The source plane $\chi^{2}$ is written as

$$
\chi_{\mathrm{S}_{i}}^{2}=\sum_{j=1}^{n_{i}} \frac{\left[\theta_{\mathrm{S}}^{j}(\mathbf{p})-\left\langle\theta_{\mathrm{S}}^{j}(\mathbf{p})\right\rangle\right]^{2}}{\mu_{j}^{-2} \sigma_{i j}^{2}},
$$

where $\theta_{\mathrm{S}}^{j}(\mathbf{p})$ is the corresponding source position of the observed image $j,\left\langle\theta_{\mathrm{S}}^{j}(\mathbf{p})\right\rangle$ is the barycenter position of all the $n_{i}$ source positions, and $\mu_{j}$ is the magnification for image $j$. Written in this way, there is no need to solve the lensing equation repeatedly and so the calculation of the $\chi^{2}$ is very fast. However, in the case where only a small number of multiple-image systems are used, source plane optimization may lead to a biased solution, typically favoring mass models with large ellipticity. 
It is important to use physically well motivated representations of the mass distribution and adjust these in order to best reproduce the different families of observed multiple images (e.g. Kneib et al. 1996; Smith et al. 2001) iteratively. Indeed, once a set of multiple images is securely identified, other multiple-image systems can in turn be discovered using morphological/color/redshift-photometric criteria, or on the basis of the lens model predictions. Better data, or data at different wavelengths may also bring new information enabling new multiple images to be identified increasing the number of constraints for modeling and hence the accuracy of mass models.

\subsubsection{Modeling the various cluster mass components}

In a cluster, the positions of multiple images are known to great accuracy and they are usually scattered at different locations within the cluster inner regions. A simple mass model with one clump cannot usually successfully reproduce observed image configurations.

We know that galaxies in general are more massive than represented by their stellar content alone. In fact, the visible stellar mass represents only a small portion (likely 10-20\%) of their total mass. The existence of an extended dark matter halo around individual galaxies has been established for disk galaxies with the measurement of their flat and spatially extended rotation curves (e.g. van Albada et al. 1985). The existence of a dark matter halo has been accepted for ellipticals only relatively recently (e.g. Kochanek et al. 1995; Rix et al. 1997). These studies found that while the stellar content dominates the central parts of galaxies, at distances larger than the effective radius the dark matter halo dominates the total mass inventory. What is less obvious in clusters of galaxies, given their dense environments, is how far the dark halos of individual early-type galaxies extend. One expects tidal "stripping" of extended dark matter halos to occur as cluster galaxies fall in and traverse through cluster cores during the assembly process. This is borne out qualitatively by the strong morphological evolution observed in cluster galaxies (e.g. Lewis et al. 2000; Kodama et al. 2002; Treu et al. 2003). In fact, lensing offers a unique probe of the mass distribution on these smaller scales within cluster environments.

The lensing effects of individual galaxies in clusters was first noted by Kassiola et al. (1992) who detected that the lengths of the triple arc in C10024+1654 can only be explained if the galaxies near the ' $\mathrm{B}$ ' image were massive enough. Detailed treatment of the individual galaxy contribution to the overall cluster mass distribution became critical with the refurbishment of the HST as first shown by Kneib et al. (1996). It was found that cluster member galaxies and their associated individual dark matter halos need to be taken into account to accurately model the observed strong lensing features in the core of Abell 2218.

The theory of what is now referred to as galaxy-galaxy lensing in clusters was first formulated and discussed in detail by Natarajan and Kneib (1997), and its application to data followed shortly (Natarajan et al. 1998; and Geiger and Schneider 1999). From their detailed analysis of the cluster AC 114, Natarajan et al. (1998) concluded that dark matter distributed on galaxy scales in the form of halos of cluster members contributes about $10 \%$ of the total cluster mass. Analysis of this effect in several cluster lenses at various redshifts seems to indicate that tidal stripping does 
in fact severely truncate the dark matter halos of infalling cluster galaxies. The dark matter halos of early-type galaxies in clusters is found to be truncated compared to that of equivalent luminosity field galaxies (Natarajan et al. 2002a, 2002b; Natarajan and Springel 2004). More recent work finds that tidal stripping is on average more efficient for late-type galaxies compared to early-type galaxies (Natarajan et al. 2009) in the cluster environment.

Lens models need to include the contribution of small scale potentials in clusters like those associated with individual cluster galaxies to reproduce the observed image configurations and positions. As there are only a finite number of multiple images, the number of constraints is limited. It is therefore important to limit the number of free parameters of the model and keep it physically motivated-as in the end-we are interested in deriving physical properties that characterize the cluster fully.

Generally, in these parametric approaches, the cluster gravitational potential is decomposed in the following manner:

$$
\phi_{\mathrm{tot}}=\sum_{i} \phi_{c_{i}}+\sum_{j} \phi_{p_{j}}
$$

where we distinguish smooth, large-scale potentials $\phi_{c_{i}}$, and the sub-halo potentials $\phi_{p_{j}}$ that are associated with the halos of individual cluster galaxies as providing small perturbations (Natarajan and Kneib 1997). The smooth cluster-scale halos usually represent both the dark matter and the intra-cluster gas. However, combining with X-ray observations, each of these two components could in principle be modeled separately. For complex systems, more than one cluster-scale halo is often needed to fit the data. In fact, this is the case for many clusters: Abell 370, 1689, 2218 to name a few.

The galaxy-scale halos included in the model represent all the massive cluster member galaxies that are roughly within two times the Einstein radius of the cluster. This is generally achieved by selecting galaxies within the cluster red sequence and picking the brighter ones such that their lensing deflection is comparable to the spatial resolution of the lensing observation. Studies of galaxy-galaxy lensing in the field have shown that a strong correlation exists between the light and the mass profiles of elliptical galaxies (Mandelbaum et al. 2006). Consequently, to a first approximation, in mass modeling the location, ellipticity and orientation of the smaller galaxy halos are matched to their luminous counterparts.

Except for a few galaxy scale sub-halos that do perturb the locations of multiple images in their vicinity or alter the multiplicity of lensed images in rare cases, the vast majority of galaxy scale sub-halos act to merely increase the total mass enclosed within the Einstein radius. In order to reduce the number of parameters required to describe galaxy scale halos, well motivated scaling relations with luminosity are often adopted. Following the work of Brainerd et al. (1996) for galaxy-galaxy lensing in the field, galaxy-scale sub-halos within clusters are usually modeled with individual PIEMD potentials. The mass profile parameters for this model are the core radius $\left(r_{\text {core }}\right)$, cut-off radius $\left(r_{\text {cut }}\right)$, and central velocity dispersion $\left(\sigma_{0}\right)$, which are scaled to 
the galaxy luminosity $L$ in the following way:

$$
\left\{\begin{array}{l}
\sigma_{0}=\sigma_{0}^{\star}\left(\frac{L}{L^{\star}}\right)^{1 / 4}, \\
r_{\text {core }}=r_{\text {core }}^{\star}\left(\frac{L}{L^{\star}}\right)^{1 / 2}, \\
r_{\text {cut }}=r_{\text {cut }}^{\star}\left(\frac{L}{L^{\star}}\right)^{\alpha} .
\end{array}\right.
$$

The total mass of a galaxy scale sub-halo then scales as (Natarajan and Kneib 1997):

$$
M=(\pi / G)\left(\sigma_{0}^{\star}\right)^{2} r_{\text {cut }}^{\star}\left(L / L^{\star}\right)^{1 / 2+\alpha},
$$

where $L^{\star}$ is the typical luminosity of a galaxy at the cluster redshift. When $r_{\text {core }}^{\star}$ vanishes, the potential becomes a singular isothermal potential truncated at the cutoff radius.

In the above scaling relations (52), the velocity dispersion scales with the total luminosity in agreement with the empirically derived Faber-Jackson relations for elliptical galaxies (for spiral galaxies the Tully-Fisher should be used instead, but those are not numerous in cluster cores). When $\alpha=0.5$, the mass-to-light ratio is constant and is independent of the galaxy luminosity, however, if $\alpha=0.8$, the massto-light ratio scales with $L^{0.3}$ similar to the scaling seen in the fundamental plane (Jorgensen et al. 1996; Halkola et al. 2006). Other scalings are of course permissible, and a particularly interesting one that has been recently explored in field galaxygalaxy lensing studies, is to scale the sub-halo mass distribution directly with the stellar mass (see Leauthaud et al. 2011).

\subsubsection{Bayesian modeling}

State of the art parametric modeling is done in the context of a fully Bayesian framework (Jullo et al. 2007), where the prior is well defined and the marginalization is done over all the relevant model parameters that represent the cluster mass distribution. Indeed, the Bayesian approach is better suited than regression techniques in situations where the data by themselves do not sufficiently constrain the model. In this case, prior knowledge about the Probability Density Function (PDF) of parameters helps to reduce degeneracies in the model. The Bayesian approach is well-suited to strong lens modeling given the few constraints generally available to optimize a model. The Bayesian approach provides two levels of inference rather efficiently: parameter space exploration, and model comparison. Bayes Theorem can be written as

$$
\operatorname{Pr}(\mathbf{p} \mid D, M)=\frac{\operatorname{Pr}(D \mid \mathbf{p}, M) \operatorname{Pr}(\mathbf{p} \mid M)}{\operatorname{Pr}(D \mid M)},
$$

where $\operatorname{Pr}(\mathbf{p} \mid D, M)$ is the posterior $\operatorname{PDF}, \operatorname{Pr}(D \mid \mathbf{p}, M)$ is the likelihood of getting the observed data $D$ given the parameters $\mathbf{p}$ of the model $M, \operatorname{Pr}(\mathbf{p} \mid M)$ is the prior PDF for the parameters, and $\operatorname{Pr}(D \mid M)$ is the evidence. 
The value of the posterior PDF will be the highest for the set of parameters $\mathbf{p}$ which gives the best fit and that is consistent with the prior PDF, regardless of the complexity of the model $M$. Meanwhile, the evidence $\operatorname{Pr}(D \mid M)$ is the probability of getting the data $D$ given the assumed model $M$. It measures the complexity of model $M$, and, when used in model selection, it acts as Occam's razor. ${ }^{5}$

Jullo et al. (2007) have implemented in LENSTOOL ${ }^{6}$ a model optimization based on a Bayesian Markov Chain Monte Carlo (MCMC) approach, which is currently widely used. Since this approach involves marginalizing over all relevant parameters, it offers a clearer picture of all the model degeneracies.

\subsection{Probing the radial profile of the mass in cluster cores}

One important prediction from dark matter only numerical simulations of structure formation and evolution in a $\Lambda \mathrm{CDM}$ Universe, is the value of the slope $\beta$ of the density profile $\rho_{\text {darkmatter }} \propto r^{-\beta}$ in the central part of relaxed gravitational systems. Although there has been on-going debate for the past decade on the exact value of the inner slope (Navarro et al. $1997(\beta=1)$; Moore et al. $1998(\beta=1.5)$ ), the real limitation of such predictions is the lack of baryonic matter in these simulations. Baryons dominate the mass budget and the gravitational potential in the inner most regions of clusters and need to be taken into account while trying to constrain the inner slope of the dark matter density profile. This is expected to change in the near future with better numerical simulations. Even in dark matter only simulations, it has been found that non-singular three-parameter models, e.g. the Einasto profile has a better performance than the singular two-parameter NFW model in the fitting of a wide range of dark matter halo structures (Navarro et al. 2010). Nevertheless, the radial slope of the total mass profile is a quantity that lensing observations can uniquely constrain (e.g. Miralda-Escudé 1995). This was first attempted for Abell 2218 by Natarajan and Kneib (1996) and subsequently by Smith et al. (2001) for the cluster Abell 383, by modeling the cluster center as the sum of a cD halo and a largescale cluster component. Abell 383 is an interesting and unique system wherein both a tangential and a radial arc with similar redshift are observed. Such a configuration provides a particularly good handle on the inner slope (here considered as the sum of the stellar and Dark Matter component), which in the case of Abell 383 was found to be steeper than the NFW prediction.

Once tangential and radial arcs have been identified from HST images (see Fig. 19), the main observational limitation is the measurement of the redshift of the multiply imaged arc to firmly constrain the radial mass profile. Large telescopes (Keck/VLT/Gemini/Subaru) have been playing a key role in cluster lensing by measuring the redshifts of multiple images. Furthermore, working at high spectral resolution allows one to also probe the dynamics of $\mathrm{cD}$ galaxies in the core of clusters (Natarajan and Kneib 1996; Sand et al. 2002). Thus combining constraints from stellar dynamics, in particular, measurements of the velocity dispersion of stars and coupling that with lensing enables the determination of the mass distribution in cluster

\footnotetext{
5 "All things being equal, the simplest solution tends to be the best one."

${ }^{6}$ LENSTOOL is available at http://lamwws.oamp.fr/lenstool/.
} 

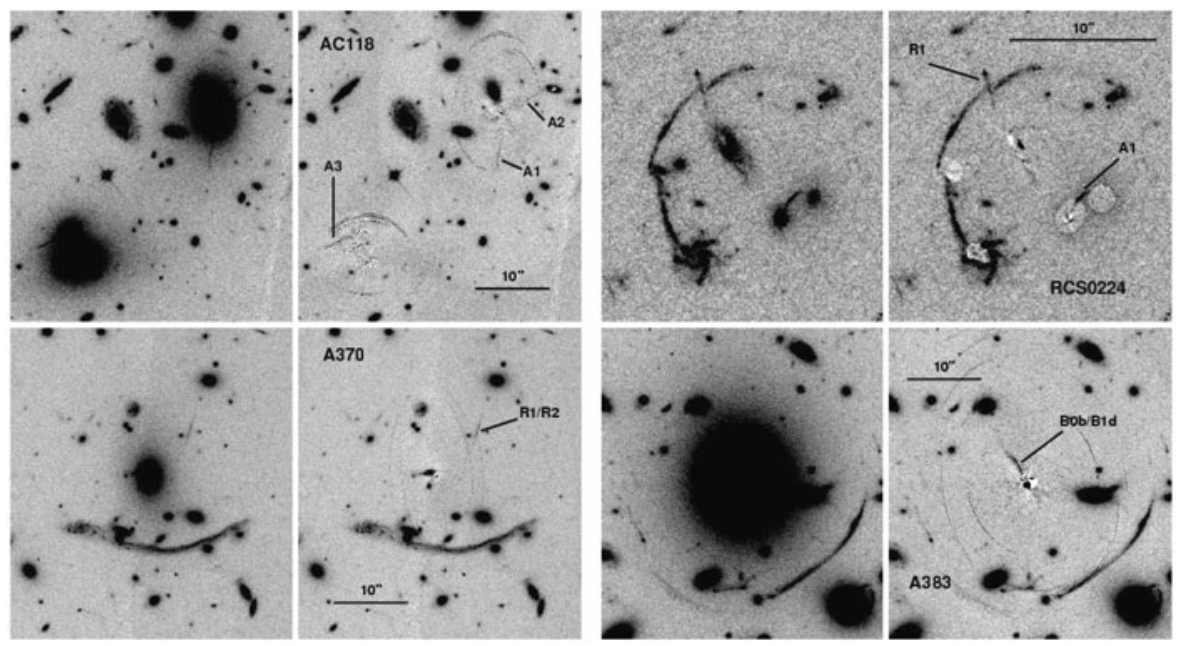

Fig. 19 Example of radial arcs found in the 4 cluster AC118, RCS0224, A370 and A383 (from Sand et al. 2005). The right side of each panel shows the BCG subtracted images

cores. This combination is very powerful as it weighs the different mass components individually: stellar mass, X-ray gas and dark matter in the cores of clusters. Sand et al. (2005) applied this technique to the clusters MS2137-23 and Abell 383 and found that the dark matter component is best described by a generalized NFW model with an inner slope that is shallower than the theoretically predicted canonical NFW profile. A similar analysis was also conducted by Gavazzi et al. (2003) with the same result. It must be stressed that the comparison between numerical simulations and observations is not direct as the stars in the $\mathrm{cD}$ galaxies dominate the total mass budget in the very center and the additional contributions of these baryons are not accounted for in the dark matter only simulations. It is widely believed that the significant presence of baryons in cluster cores likely modifies the inner density profile slope of dark matter, although there is disagreement at present on how significant this adiabatic compression is likely to be (Blumenthal et al. 1986; Gnedin et al. 2004; Zappacosta et al. 2006). Radial arcs offer a unique and possible only handle to probe the inner slopes of density profiles. Several other clusters with radial arcs have been discovered from HST archives recently, and are currently being followed up spectroscopically (Sand et al. 2005, 2008).

In a recent paper, Newman et al. (2011) have obtained high accuracy velocity dispersion measurements for the $\mathrm{cD}$ galaxy in Abell 383 out to a radius of $\sim 26 \mathrm{kpc}$ for the first time in a lensing cluster. Adopting a triaxial dark matter distribution, an axisymmetric dynamical model and using the constraints from both strong and weak lensing, they demonstrate that the logarithmic slope of the dark matter density at small radii is $\beta<1.0$ (95\% confidence), shallower than the NFW prediction (see Fig. 20). Similar analysis of other relaxed clusters, including constraints from small to large scales will help improve our understanding of the mass distribution in cluster cores and help test the assumptions used in numerical simulations where both dark matter and baryonic matter (stars and X-ray-gas) are explicitly included. 


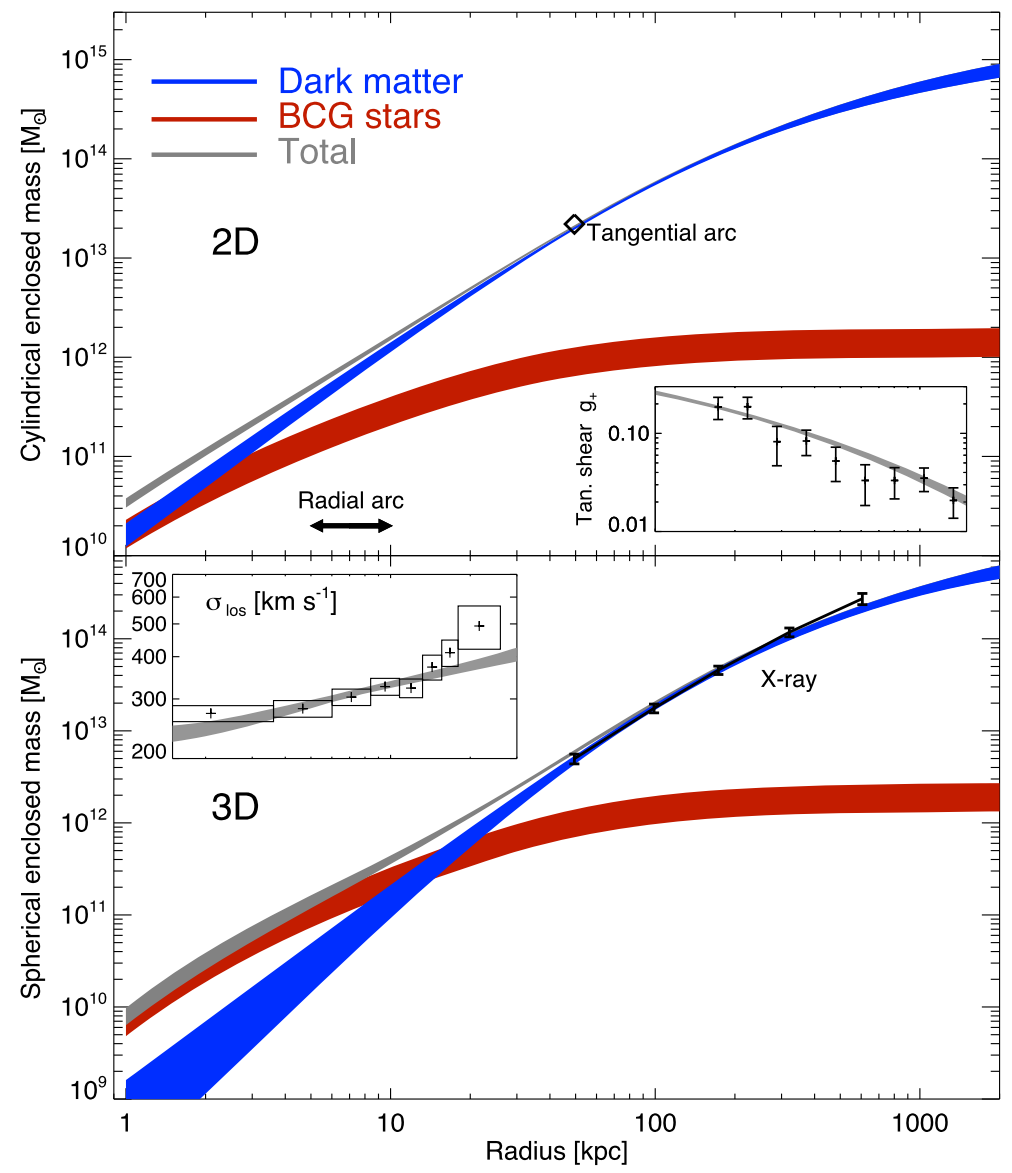

Fig. 20 Top: Projected mass for the dark matter and stellar components, as well as the total mass distribution, with tangential reduced shear $(\mathrm{g})$ data inset at the same radial scale for the cluster Abell 383. Bottom: 3D Mass, with velocity dispersion data inset and X-ray constraints overlaid. All bands show $68 \%$ confidence regions. The models acceptably fit all constraints ranging from the smallest spatial scales $\simeq 2 \mathrm{kpc}$ to $\simeq 1.5 \mathrm{Mpc}$. This figure is taken from Newman et al. (2011)

\subsection{Non-parametric strong lensing modeling}

In addition to the use of the parametric analytic mass models described above, there has been considerable progress in developing non-parametric mass reconstruction techniques in the past decade (e.g. Abdelsalam et al. 1998a, 1998b; Saha and Williams 1997; Diego et al. 2005a, 2005b; Jullo and Kneib 2009; Coe et al. 2010; Zitrin et al. 2010). Non-parametric cluster mass reconstruction methods have become more popular with the increase in available observational constraints from the numerous multiple images that are now more routinely found in deep HST data (e.g. Broadhurst et al. 2005). Non-parametric models have increased flexibility which allows a more comprehensive exploration of allowed mass distributions. These schemes are 
particularly useful to model extremely complex mass distributions such as the "Bullet Cluster" (Bradač et al. 2005).

Contrary to the analytic profile driven"parametric" methods, in "non-parametric" schemes, the mass distribution is generally tessellated into a regular grid of small mass elements, referred often to as pixels (Saha and Williams 1997; Diego et al. 2005a). Alternatively, instead of starting with mass elements, Bradač et al. (2005) prefer tessellating the gravitational potential because its derivatives directly yield the surface density and other important lensing quantities that can be related more straightforwardly to measurements. Pixels can also be replaced by radial basis functions (RBFs) that are real-valued functions with radial symmetry. Several RBFs for density profiles have been tested so far. Liesenborgs et al. (2007) use Plummer profiles, and Diego et al. (2007) use RBFs with Gaussian profiles. The properties of power law profiles, isothermal profiles as well as Legendre and Hermite polynomials have been explored as RBFs. These studies find that the use of compact profiles such as the Gaussian or Power law profiles are generally preferred as they are more accurate in reproducing the surface mass density.

In more recent work, instead of using a regular grid, Coe et al. $(2008,2010)$ and Deb et al. (2008) use the actual distribution of images as an irregular grid. Then, they either place RBFs on this grid or directly estimate the derivatives of the potential at the location of the images. Whatever their implementation, the reproduction of multiple images is generally greatly improved with respect to traditional "parametric" modeling with these techniques. However, the robustness of these models is still a matter of debate given the current observational constraints available from data. Indeed, due to the large amount of freedom that inevitably goes with the large degree of flexibility afforded by a "non-parametric" approach, many models can fit perfectly the data and discriminating between models is challenging. To identify the best physically motivated model and eventually learn more about the dark matter distribution in galaxy clusters, additional external criteria (e.g. mass positivity) or regularization terms (e.g. to avoid unwanted high spatial frequencies) are necessary. Furthermore, galaxy mass scales are usually not taken into account in these nonparametric schemes, despite the fact that successful parametric modeling has clearly demonstrated that these smaller scale mass clumps do significantly affect the positions of observed multiple images. This is a key limitation of most of the "nonparametric" models.

Jullo and Kneib (2009) [JK09 hereafter] have proposed a novel modeling scheme that includes both a multi-scale grid of RBFs and a sample of analytically defined galaxy-scale dark matter halos, thus allowing combined modeling of both complex large-scale mass components and galaxy-scale halos. In this hybrid scheme, similar to the one adopted by Diego et al. (2005a), JK09 define a coarse multi-scale grid from a pixellated input mass map and recursively refine it in the densest regions. However, in contrast to previous work, they start from a hexagonal grid (composed of triangles), on the grounds that it better fits the generally roundish shapes of galaxy clusters. For their RBF, they use truncated circular isothermal mass models, and truncated PIEMD models to explicitly include galaxy-scale halos. Thus both components are modeled with similar analytical functions which permits a simple combination for ready incorporation into the Bayesian MCMC optimization scheme built into LENSTOOL. Figure 21 shows the derived S/N convergence map for the model of the cluster A1689. 
Fig. 21 Map of $\mathrm{S} / \mathrm{N}$ ratio for the A1689 mass reconstruction using the JK09

"non-parametric" mass reconstruction. Colored contours bound regions with $\mathrm{S} / \mathrm{N}$ greater than 300, 200, 100 and 10. The highest $\mathrm{S} / \mathrm{N}$ region is at the center where there are the most constraints. Red contours are mean iso-mass contours. Black boxes mark the positions of the multiple images used to constrain the mass distribution, and the numbers indicate the different multiple-image systems (from JK09)

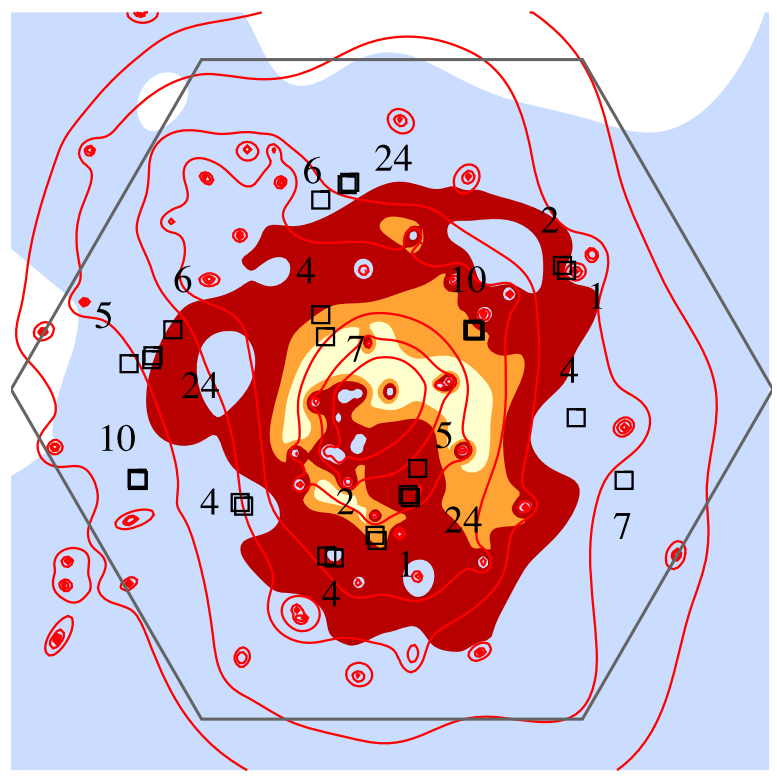

As the $\mathrm{S} / \mathrm{N}$ of the mass reconstruction is found to be larger than 10 everywhere inside the hexagon, the error in the convergence derived mass is less than $10 \%$, demonstrating the power of this hybrid scheme.

\subsection{Cluster weak lensing modeling}

As soon as we look a little bit further out radially from the cluster core, the lensing distortion gets smaller (distortions in shape get to be of the order of a few percent at most), and very quickly the shape of faint galaxies gets dominated by their intrinsic ellipticities (the dispersion of the intrinsic ellipticity distribution of observed galaxies $\left.\sigma_{\epsilon} \sim 0.25\right)$. Thus the lensing distortion is no longer visible in individual images and can only be probed in a statistical fashion, characteristic of the weak lensing regime (e.g. Bartelmann 1995). The nature of constraints provided by observations are fundamentally different in the weak lensing regime compared to the strong lensing regime. In the strong regime, as we shown above every set of observed multiple images provides strong constraints on the mass distribution. In the weak regime, however, what is measured are the mean ellipticities and/or the mean number density of faint galaxies in the frame. In order to relate these to the mean surface mass density $\kappa$ of the cluster, these data need to be used statistically. There are two key sets of challenges in doing so:

- Observational: How to best determine the 'true' ellipticity of an observed faint galaxy image which is smeared by a PSF of comparable size, and is not circular (as a result of camera distortions, variable focus across the image, tracking and guiding errors) and not stable in time? How best to estimate and isolate the variation in the number density of faint galaxies due to lensing, while taking into account the crowding effect due to the presence of cluster members and the intrinsic spatial fluctuations in the distribution of galaxies; and the unknown redshift distribution of background sources? 
- Theoretical: what is the optimal method to reconstruct the surface mass density distribution $\kappa$ (as a mass map or a radial mass profile) using either the 'reduced shear field' $\vec{g}$ and/or the amplification?

Various approaches have been proposed to solve these sets of problems, and two distinct families of methods can be distinguished: direct and inverse methods. We describe them in detail in what follows.

\subsubsection{Weak lensing observations}

For observers, before any data handling, the first step is to choose the telescope (and instrument) that will minimize the sources of noise in the determination of the ellipticity of faint galaxies. Although HST has the best characteristics in terms of the PSF size, it has a very limited field of view $\sim 10$ square arcminutes for the ACS camera. Furthermore, Hubble is "breathing" as it is orbiting around the Earth, which affects the focus of its instruments, and the most recent ACS imaging data appear to be suffering from Charge Transfer Inefficiency (CTI) which needs special post-processing for removal of these instrumental effects (Massey 2010). Although, as we have seen, HST is ideal for picking out strong lensing features (e.g. Gioia et al. 1998), it is not the most appropriate instrument to probe the large-scale distribution of a cluster extending out to and beyond the virial radius. Note, however, this limitation becomes less of a problem when observing high-redshift clusters (e.g. Hoekstra et al. 2002; Jee et al. 2005; Lombardi et al. 2005).

On the ground a number of wide-field imaging cameras have been used to conduct weak lensing measurements in cluster fields. The most productive ones in the last decades have been: the CFHT12k camera (e.g. Bardeau et al. 2007; Hoekstra 2007) and the more recent Megacam camera (e.g. Gavazzi and Soucail 2007, Shan et al. 2010) at CFHT, and the Suprimecam on Subaru (e.g. Okabe et al. 2010). However, a number of studies have also been done with other instruments, in particular, the VLT/FORS (e.g. Cypriano et al. 2001, Clowe et al. 2004a, 2004b), 2.2 m/WFI (e.g. Clowe and Schneider 2002), and more recently with the LBT camera (Romano et al. 2010).

\subsubsection{Galaxy shape measurement}

Once the data have been carefully taken either from the ground or space with utmost care to minimize contaminating distortions and hopefully under the best seeing conditions, the next step is to convert the image of the cluster into a catalog where the PSF corrected shapes of galaxies are computed.

Before measuring the galaxy shape a number of steps are usually undertaken: (1) masking of the data and identifying the regions in the image that suffer from observational defects: bleeding stars, satellite tracks, hot pixels, spurious reflections; (2) source identification and catalog production-which is usually done using the SEXTRACTOR software (Bertin and Arnouts 1996); (3) identification of the stellar objects-in order to accurately compute the PSF as a function of position in the image. Once this is done, the shapes of the stellar objects and galaxies can be computed to derive the PSF corrected shapes of galaxies which will then be used for weak lensing measurements. For accomplishing this crucial next 
step, a popular and direct approach is often used, to convert galaxy shapes to shear measurements using the IMCAT software package. This implementation is based on the Kaiser et al. (1995) methodology, but has been subsequently improved by various other groups (e.g. Luppino and Kaiser 1997; Rhodes et al. 2000; Hoekstra et al. 2000), providing variants of the original KSB technique. To correct the galaxy shape from the PSF anisotropy and circularization, the KSB technique uses the weighted moment of the object's surface brightness to find its center, and shape to measure higher order components that can be used to improve the PSF correction. As a result the correction is fast and processing a large amount of data can be done swiftly and efficiently.

Alternatively, one can use the inverse approach using maximum likelihood methods or Bayesian techniques to find the optimal galaxy shape that when convolved with the local PSF best reproduces the observed galaxy shape (e.g. Kuijken 1999; IM2SHAPE: Bridle et al. 2002). A recent implementation of this inverse method is available in the LENSFIT software package (Miller et al. 2007; Kitching et al. 2008) and one of its key advantages is that it works directly on the individual exposures of a given field. LENSFIT has been developed in the context of the CFHT-LS survey, but is flexible and can be easily adapted for use with other observations.

These inverse approaches have the advantage that they provide a direct estimate of the uncertainty in the parameter recovery as illustrated in Fig. 22. Further extension of these inverse techniques, has led to the use of SHAPELETS (Refregier 2003; Refregier and Bacon 2003) that offer a more sophisticated basis set to characterize the two-dimensional shapes of the PSF and faint galaxies. The versatility of shapelets has made this technique quite popular for lensing measurements. Nevertheless, it has been realized that these different shape measurement recipes need to be tuned, compared and calibrated amongst each other in order to obtain accurate, unbiased and robust shear measurements. This calibration work has been done in the context of various numerical challenges, wherein different research groups measure the shapes of the same set of simulated images as part of STEP (Heymans et al. 2006; Massey et al. 2007); the GREAT08 and GREAT10 challenges (Bridle et al. 2010; Kitching et al. 2011). These challenges have proven to be very useful exercises for the community as they have enabled calibration of the several independent techniques employed to derive shear from observed shapes.

\subsubsection{From galaxy shapes to mass maps}

From the catalog of shape measurements of faint galaxies, a mass map can be derived. And here again direct and inverse methods have been explored. The direct approaches are: (i) the Kaiser and Squires (1993) method-this is an integral method that expresses $\kappa$ as the convolution of $\vec{\gamma}$ with a kernel and subsequent refinements thereof (e.g. Seitz and Schneider 1995, 1996; Wilson et al. 1996); and (ii) a local inversion method (Kaiser 1995; Schneider 1995; Lombardi and Bertin 1998) that involves the integration of the gradient of $\vec{\gamma}$ within the boundary of the observed field to derive $\kappa$. This technique is particularly relevant for datasets that are limited to a small field of view.

The inverse approach works for both the $\kappa$ field and the lensing potential $\varphi$ and uses a maximum likelihood (e.g. Bartelmann et al. 1996; Schneider et al. 2000; 

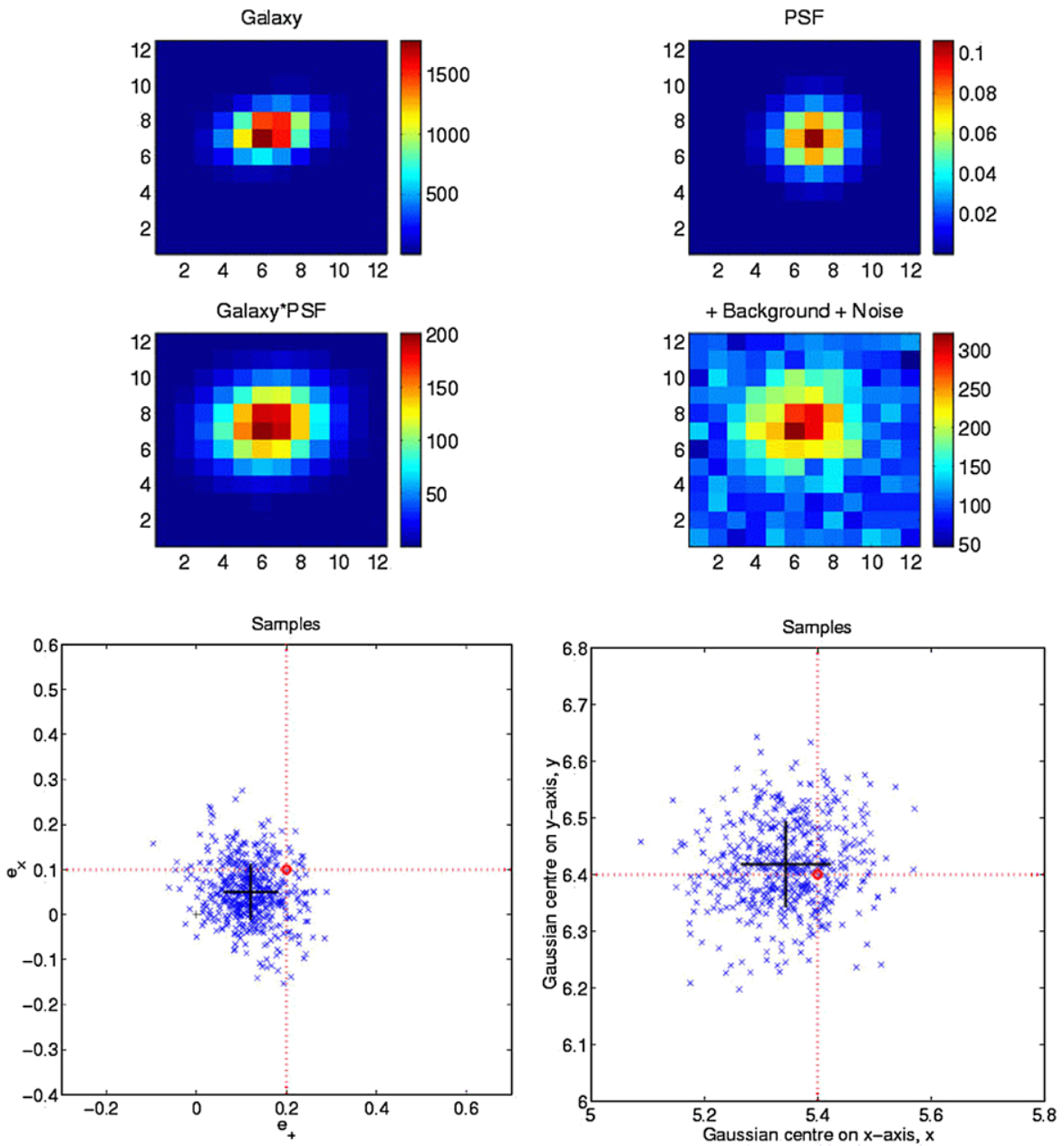

Fig. 22 Simulated images demonstrating the various sources of noise in weak lensing data: the galaxy model, the PSF model, the convolution of the two, and the final image when noise is added. Simple simulations allow the production of model images that include observational sources of noise and can therefore be compared directly to the shapes of observed faint galaxies. The best galaxy model is then found by taking into account the convolution effect of the measured PSF that best fits the data. (Bottom panels): the MCMC samples fitting the final image in terms of its ellipticity vector with coordinates $\left[e_{+}=e_{1}\right]$ and $\left[e_{\times}=e_{2}\right]$ (on the left) and in terms of the position $(x, y)$ of the image center (right), from which the best-fit fiducial models with errors can be extracted (black crosses). One of the first implementations of this inverse technique was done in the IM2SHAPE software (Bridle et al. 2002)

King and Schneider 2001), maximum entropy method (e.g. Bridle et al. 1998; Marshall et al. 2002) or atomic inference approaches coupled with MCMC optimization techniques (Marshall 2006) to determine the most likely mass distribution (as a 2D mass map or a 1D mass profile) that reproduces the reduced shear field $\vec{g}$ and/or the variation in the faint galaxy number densities. These inverse methods are of great interest as they enable quantifying the errors in the resultant mass maps or mass esti- 

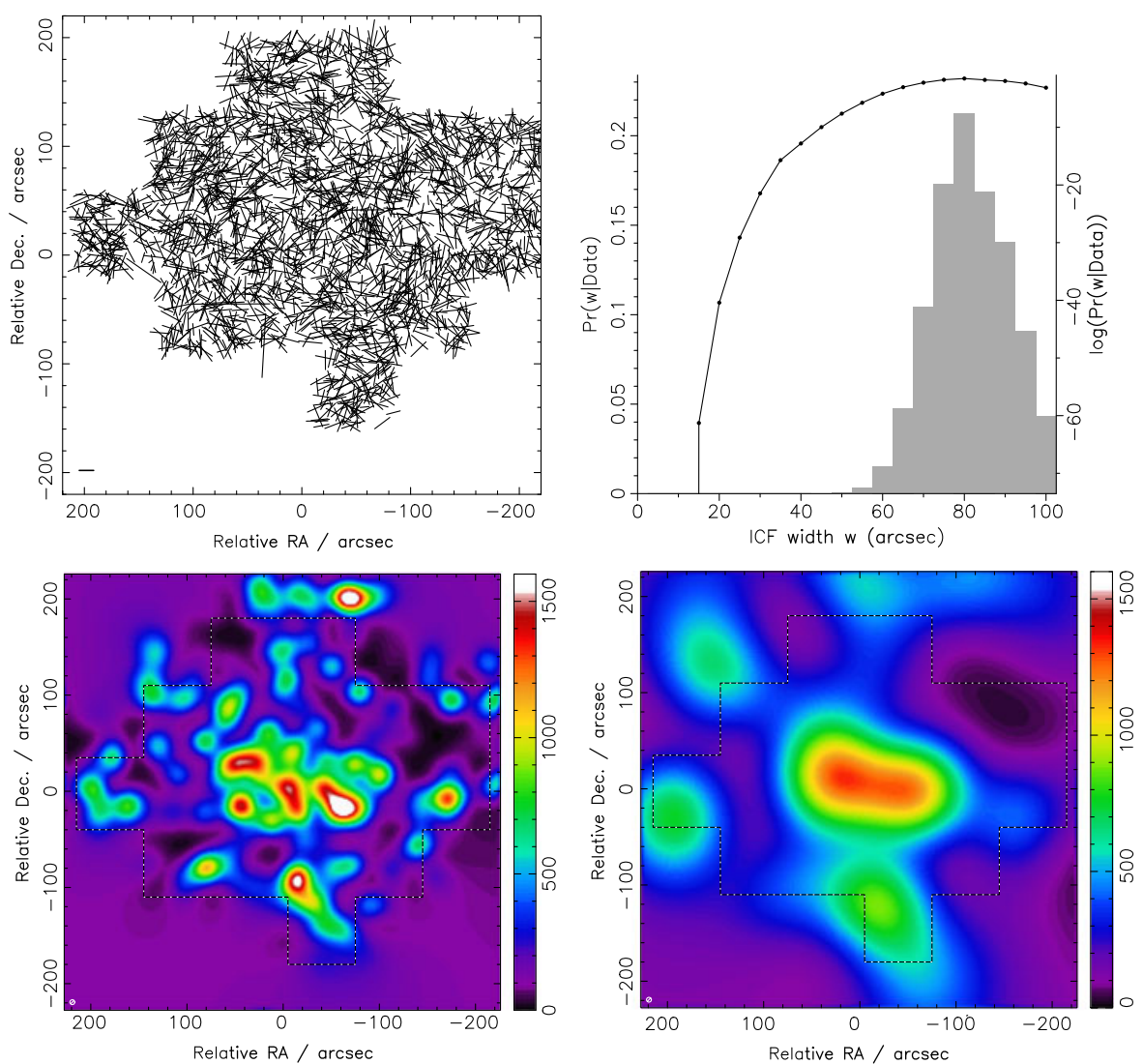

Fig. 23 Maximum entropy mass reconstruction (Marshall et al. 2002; Marshall 2003) of the X-ray luminous cluster MS1054 at $z=0.83$ using Hoekstra's HST dataset (Hoekstra et al. 2000). (Top left) Distribution of the positions of galaxies used in the mass reconstruction. (Top right) Evidence values for different sizes of the Intrinsic Correlation Function (ICF). (Bottom) Two mass reconstructions illustrate the case of 2 different values for the ICF: (left) small ICF with a low evidence value, (right) large ICF with the largest evidence

mates (e.g. Kneib et al. 2003), and in principle, can cope with the addition of further external constraints from strong lensing or X-ray data simultaneously. Wavelet approaches that use the multi-scale entropy concept have also been extremely powerful in producing multi-scale mass maps (Starck et al. 2006; Pires et al. 2009).

An important issue for producing mass maps is the resolution at which the $2 \mathrm{D}$ lensing mass map can be reconstructed. Generally, mass maps are reconstructed on a fixed size grid, which then automatically defines the minimum mass resolution that can be obtained. By comparing the likelihood of different resolution mass maps, we can calculate the Bayesian evidence of each to determine the optimal resolution (Fig. 23). However, it is most likely that the optimal scale to which a mass map can be reconstructed is adaptive, and is determined by the strength of the lensing signal. As we are limited by the width of the intrinsic ellipticity distribution, it is only by averaging over a large number of galaxies that we can reach lower shear levels. Thus low shear levels can only be probed on relatively large scales by averaging over a large number 


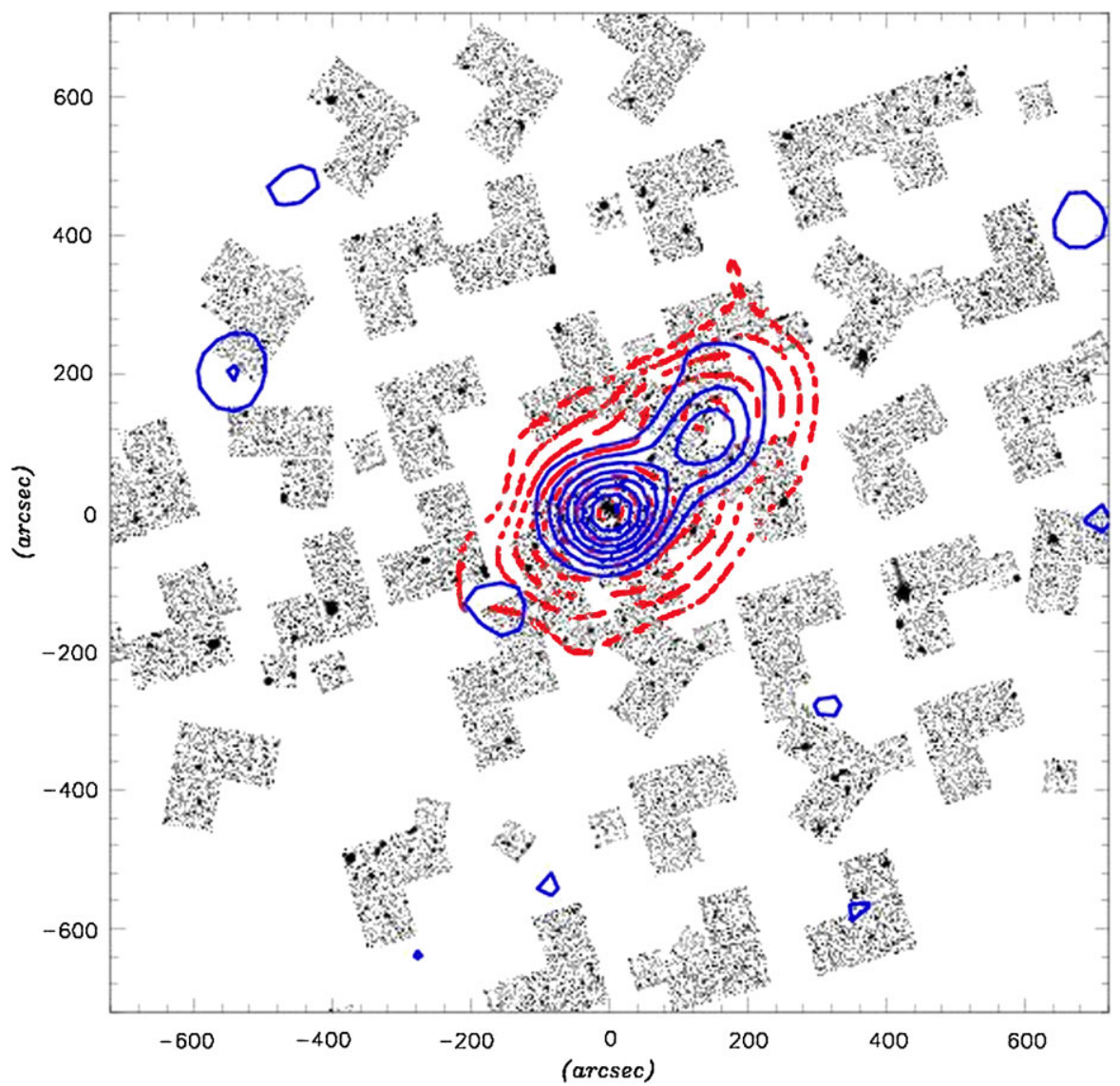

Fig. 24 The 39 WFPC2/F814W, and the 38 STIS/50CCD pointings sparsely covering the C10024+1654 cluster. The (red) dashed contours represent the number density of cluster members as derived by Czoske et al. (2001). The blue solid contour is the mass map built from the joint WFPC2/STIS analysis derived using the LensEnt software (Bridle et al. 1998; Marshall et al. 2002)

of galaxies. Furthermore, as the projected surface mass density of clusters on large scales falls off relatively quickly scaling as $1 / R$ to $1 / R^{2}$, respectively, for an SIS or a NFW profile, mass maps may quickly lose spatial resolution.

Although lensing mass maps may quickly loose information content outside the cluster core, they can be very useful in identifying possible (unexpected) substructures on scales larger than the typical weak lensing smoothing scale ( $\sim 1$ arcminute). This has been the case for several cluster lenses such as the cluster C10024+1654 (e.g. Kneib et al. 2003 and Fig. 23; Okabe et al. 2010) and the "Bullet Cluster" (see Fig. 25), and more recently in the so-called "baby bullet" cluster (Bradač 2009).

\subsubsection{Measuring total mass and mass profiles}

Weak lensing mass maps are useful to determine mass peaks, but they are of limited use for the extraction of physical information. If a cluster has a relatively simple 


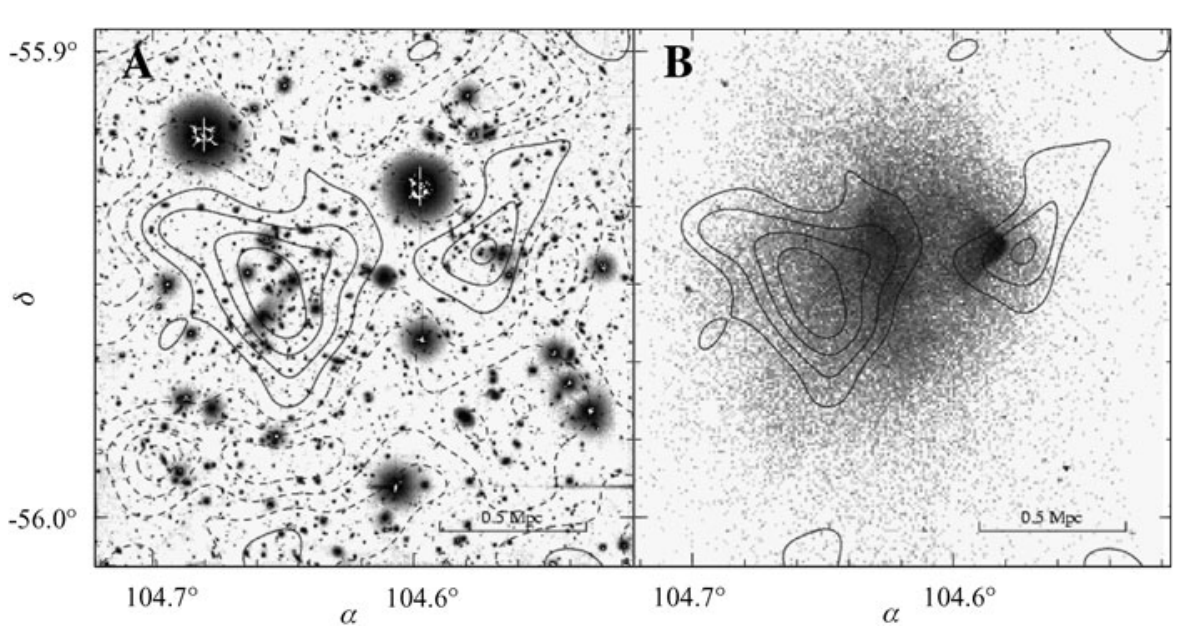

Fig. 25 Right panel: Shown in greyscale is the $I$-band VLT/FORS image of the Bullet cluster used to measure galaxy shapes. Over-plotted in black contours is the weak lensing mass reconstruction with solid contours for positive mass, dashed contours for negative mass, and the dash-dotted contour for the zero-mass level. Left panel: Shown in greyscale is the Chandra X-ray image from Markevitch et al. (2002) with the same weak lensing contours as in the Right Hand panel. (Figure from Clowe et al. 2004a, 2004b)

geometry (i.e. has a single mass clump in its $2 \mathrm{D}$ mass map) one can easily extract the radial mass profile and compute the total mass enclosed within a given radial aperture. Different approaches are currently available to compute the mass profile and the total mass. The direct method is just to sum up the tangential weak shear as expressed in the aperture mass densitometry first introduced by Fahlman et al. (1994) and then revised by Clowe et al. (1998). This statistic is quite popular and has been used in several recent cluster modeling papers, including Hetterscheidt et al. (2005), Hoekstra (2007) and Okabe et al. (2010). Aperture mass densitometry measures the mass interior to a given radius following the $\zeta$ statistic, defined as

$$
\zeta\left(\theta_{1}\right)=\bar{\kappa}\left(\theta<\theta_{1}\right)-\bar{\kappa}\left(\theta_{1}<\theta<\theta_{\max }\right)=2\left(1-\frac{\theta_{1}^{2}}{\theta_{\max }^{2}}\right) \int_{\theta_{1}}^{\theta_{\max }}\left\langle\gamma_{\mathrm{T}}\right\rangle d \ln \theta,
$$

which provides a lower bound on the mean convergence $\bar{\kappa}$ interior to radius $\theta_{1}$. The mass within $\theta$ is then just given by

$$
M(<\theta)=\pi D_{\mathrm{OL}}^{2} \theta^{2} \Sigma_{\text {crit }} \zeta(\theta) .
$$

This statistic assumes, however, that all background galaxies are at a similar redshift, which can be a strong and severely limiting assumption, particularly for high-redshift clusters.

Another semi-direct approach is to build the projected surface density contrast $\Delta \Sigma$ estimator as introduced by Mandelbaum et al. (2005):

$$
\Delta \Sigma(r)=\bar{\Sigma}(<r)-\Sigma(r)=\gamma_{\mathrm{T}}(r) \Sigma_{\text {crit }}\left(z_{\mathrm{S}}\right) .
$$


In practice, $\Delta \Sigma$ is measured by averaging over the galaxies at radius $r$ from the cluster center, and requires some information about the redshift distribution of background galaxies $z_{\mathrm{S}}$. It can then be directly compared to the $\Delta \Sigma(r)$ computed for a given parametrized mass model. Mandelbaum et al. (2010) discuss and compare this cluster mass estimator with other proposed ones. In a recent paper, Gruen et al. (2011) compare the use of various aperture mass estimators to calibrate mass-observable relations from weak lensing data.

The alternative method is to directly fit the observables using simple parametric models similar to what is done in the strong lensing approach, for example using radially binned data (e.g. Fischer and Tyson 1997; Clowe and Schneider 2002; Kneib et al. 2003; King et al. 2002). The advantage of adopting such a method lies in its flexibility, i.e. allowing combination of strong and weak lensing constraints. This direct approach also allows inclusion of external constraints such as those from Xray data and the redshift distribution of background sources that can be estimated using photometric-redshift techniques. Of course, such parametric techniques require allowing sufficient freedom in the radial profile and the inclusion of substructures (e.g. Metzler et al. 1999, 2001; King et al. 2001) to closely match observed lensing distortions.

\subsection{Cluster triaxiality}

As lensing is sensitive to the integrated mass along the line of sight, it is natural to expect mass overestimates due to fortuitous alignment of mass concentrations not physically related to the cluster or alternatively departures of the cluster dark matter halo from spherical symmetry (e.g. Gavazzi 2005). Till recently, most studies of the dark matter distribution and the intra-cluster medium (ICM) in galaxy clusters using $\mathrm{X}$-ray data have been limited due to the assumption of spherical symmetry. However, the Chandra and XMM-Newton X-ray telescopes have resolved the core of the clusters, and have detected departures from isothermality and spherical symmetry. Evidence for a flattened triaxial dark matter halo around five Abell clusters had been reported early on by Buote and Canizares (1996). Furthermore, numerical simulations of cluster formation and evolution in a cold dark matter dominated Universe do predict that dark matter halos have highly elongated axis ratios (Wang and White 2009), disproving the assumption of spherical geometry. In fact the departures from sphericity of a cluster may help explain the discrepancy observed between cluster masses determined from X-ray and strong lensing observations (Gavazzi 2005). This suggests that clusters with observed prominent strong lensing features are likely to be typically preferentially elongated along the line of sight which might account for their enhanced lensing cross sections. This is definitely the case for the extreme strong lenses with large Einstein radii and therefore anomalously high concentrations. The galaxy cluster A1689 is a well-studied example with such a mass discrepancy (Andersson and Madejski 2004; Lemze et al. 2008; Riemer-Sorensen et al. 2009; Peng et al. 2009). In the same vein, large values of the NFW model concentration parameters have also been reported for clusters with prominent strong lensing features (Comerford and Natarajan 2007; Oguri et al. 2009). This can again be explained by strong lensing cluster halos having their major axis preferentially oriented toward the line of sight (Corless et al. 2009). 
Combining strong lensing constraints with high-resolution images of cluster cores in X-rays obtained with Chandra is an excellent way to probe the triaxiality of the mass distribution in cluster cores. Mahdavi et al. (2007) provided a new framework for the joint analysis of cluster observations (JACO) using simultaneous fits to X-ray, Sunyaev-Zel'dovich (SZ), and weak lensing data. Their method fits the mass models simultaneously to all data, provides explicit separation of the gaseous, dark, and stellar components, and allows joint constraints on all measurable physical parameters. The JACO prescription includes additional improvements to previous X-ray techniques, such as the treatment of the cluster termination shock and explicit inclusion of the BCG's stellar-mass profile. Upon application of JACO to the rich galaxy cluster Abell 478 they report excellent agreement among the X-ray, lensing, and SZ data.

Morandi et al. (2010) have used a triaxial halo model for the galaxy cluster MACS J1423.8+2404 to extract reliable information on the 3D shape and physical parameters, by combining X-ray and lensing measurements. They found that this cluster is triaxial with dark matter halo axial ratios $1.53 \pm 0.15$ and $1.44 \pm 0.07$ on the plane of the sky and along the line of sight, respectively. They report that such a geometry produces excellent agreement between the X-ray and lensing mass.

These first results are very encouraging and pave the way for a better understanding of the 3D distribution of the various mass constituents in clusters. Theoretically, according to the current dark matter dominated cosmological model for structure formation cluster halo shapes ought to be triaxial and a firm prediction is proffered for the distribution of axis ratios for clusters. More observational work needs to be done to test these predictions, and ultimately techniques that combine lensing, X-ray and Sunyaev-Zel'dovich decrement data might be able to provide a complete threedimensional view of clusters.

\section{Mass distribution of cluster samples}

Although the careful modeling of individual cluster cores and extended regions offers a unique way to characterize the mass distribution and understand cluster physics in detail, analysis of cluster samples provides important insights into cluster assembly and evolution. There have been several statistical studies focused on measuring cluster masses derived from lensing and comparing these with mass estimates from other measurements such as: richness, X-ray luminosity, X-ray temperature, velocity dispersions of cluster galaxies, and the Sunyaev-Zel'dovich decrement. These multi-wavelength comparisons enable a deeper understanding of empirically derived scaling relations between key physical properties of clusters (e.g. Luppino and Gioia 1992; Loeb and Mao 1994; Miralda-Escudé and Babul 1995; Allen 1998; Ota et al. 1998; Ono et al. 1999; Irgens et al. 2002; Huterer and White 2002). These studies also help uncover how mass is partitioned between the different baryonic and non-baryonic components on cluster scales. Studying cluster samples allows the probing of several fundamental questions with regard to the dynamical state of clusters, namely, are clusters relaxed? How much substructure is present in clusters? How triaxial are clusters? How recently has a cluster had a major merger with another subcluster and what are the signatures of such an event? How important are projection 
effects in mass estimates? Are clusters in hydrostatic equilibrium? When did clusters start to assemble? And how have they evolved? Observationally derived answers to these questions from cluster samples can then be directly compared to numerical simulations, thus providing insights and tests of the structure formation paradigm.

\subsection{Early work}

Comprehensive multi-wavelength datasets that ideally span a range of spatial scales in clusters are needed for such statistical studies. Collecting such datasets is a big challenge as it requires coordination between researchers working with a range of observational techniques deploying many different resources. Some of the first studies of cluster samples did produce interesting cosmological results, as discussed in Luppino et al. (1999), Allen et al. (2001, 2002), Dahle et al. (2002) and Smith et al. (2003).

One of the key challenges for these statistical studies lies in the very definition of a sample with robust criteria that will be complete and volume limited and be representative to avoid systematic biases. Starting from simple selection criteria is therefore very important. For instance, dramatic lensing clusters imaged by HST are likely a biased sample of the most massive clusters at any redshift with enhanced strong lensing cross sections due to an excess of mass along the line of sight from either the cluster itself or the presence of other intervening structures. Most frequently cluster samples are therefore selected on the basis of their X-ray luminosities, which should minimize projection effects that typically plague optically selected clusters. Since X-ray luminosity is proportional to the square of the electron density of the Intra-Cluster-Medium (ICM), this selection should pick genuinely virialized clusters, irrespective of the line of sight distribution of cluster member galaxies or additional background structures. One of the first systematic studies that combined X-ray and lensing data was a sample of $12 z \sim 0.2 \mathrm{X}$-ray luminous clusters of galaxies selected from the XBACS catalog (see Figs. 26 and 27) with $L_{X}>8 \times 10^{44} \mathrm{erg} / \mathrm{s}$ in the 0.1-2.4 keV band. These clusters have been imaged with the WFPC2 camera (Smith et al. 2001, 2005). It is found that the fraction of strong lensing clusters in this sample is $70 \%$. All of the cluster cores also have a significant weak lensing signal, providing independent lensing constraints on cluster masses.

Smith et al. (2005) defined a number of criteria to characterize whether clusters are relaxed and also quantified the amount of substructure in them. Out of 10 clusters, they found that three clusters form a homogeneous sub-sample that have mature, undisturbed gravitational potentials which satisfy the following criteria: a dominant central dark matter halo $\left(M_{\mathrm{cen}} / M_{\mathrm{tot}}>0.95\right)$; a dominant central cluster galaxy $\mathrm{K}$ band luminosity fraction $\left(L_{\mathrm{K}, \mathrm{BCG}} / L_{\mathrm{K}, \text { tot }}>\sim 0.5\right)$; close alignment between the center of the mass distribution and the peak of the X-ray flux $\left(\Delta r_{\text {peak }}<3 \mathrm{kpc}\right)$; a single cluster-scale dark matter halo best fit for the lens model; and circular or mildly elliptical X-ray flux contours. The remaining seven clusters did not satisfy one or more of these criteria and were classified as disturbed. The disturbed clusters are much more diverse than the undisturbed clusters and typically have a bi- or tri-modal dark matter distribution, irregular X-ray morphology and an offset between X-ray and mass peaks. Comparison of these results with theoretical predictions indicates that the multi-modal dark matter distribution in disturbed clusters is due to recent 

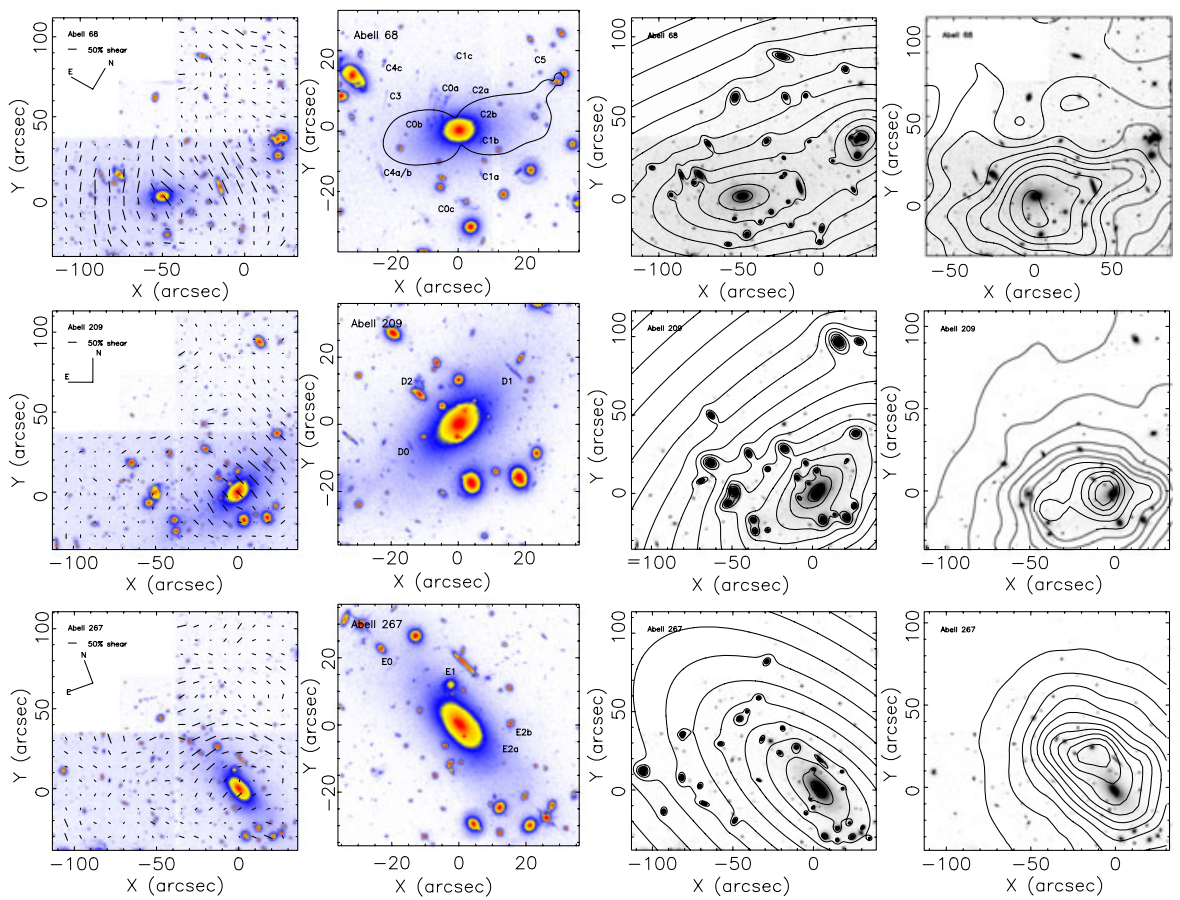

Fig. 26 Cluster samples: 3 of the $12 z \sim 0.2 \mathrm{X}$-ray luminous clusters of galaxies selected from the XBACS catalog (Ebeling et al. 1996) observed with the HST/WFPC2 camera. Top row is Abell 68, second row is Abell 209 and last row Abell 267. First column is the weak shear field as measured from the HST data. The second column is a zoom of the cluster cores, and shows for Abell 68 the predicted critical lines (black lines). The third column is the strong lensing mass reconstruction and last column is the overlay of the Chandra X-ray map (Smith et al. 2003)

infall of galaxy groups into the parent cluster since about $z=0.4$. The exact scaling relation between lensing mass and X-ray properties appears to be strongly dependent on the dynamical state of the cluster. Relaxed and unrelaxed clusters appear to follow slightly different scaling relations. Furthermore, this sample was also observed with the wide field CFHT12k camera in three bands $(\mathrm{B}, \mathrm{R}, \mathrm{I})$ in order to probe the wide field mass distribution using the measured weak lensing shear signal out to the virial radius. However, the comparison of the weak lensing determined mass to the cluster luminosity and X-ray mass estimates reported in Bardeau et al. (2007) (see Fig. 28) does not reveal an obvious difference between relaxed or unrelaxed clusters. There are some strong limitations though with this dataset as there were scant constraints on the redshift distribution of background sources, and some lingering inconsistencies between strong and weak lensing results. These first results with only 10 clusters set the stage for the need for larger cluster samples to understand the physical origin of such differences.

In a parallel paper, Hoekstra (2007) investigated the lensing versus X-ray mass relations for a sample of 20 clusters including those of Bardeau et al. (2007), although their cluster selection was primarily driven by X-ray emission. This investigation has lead to a more ambitious project known as the Canadian Cluster Comparison Project 

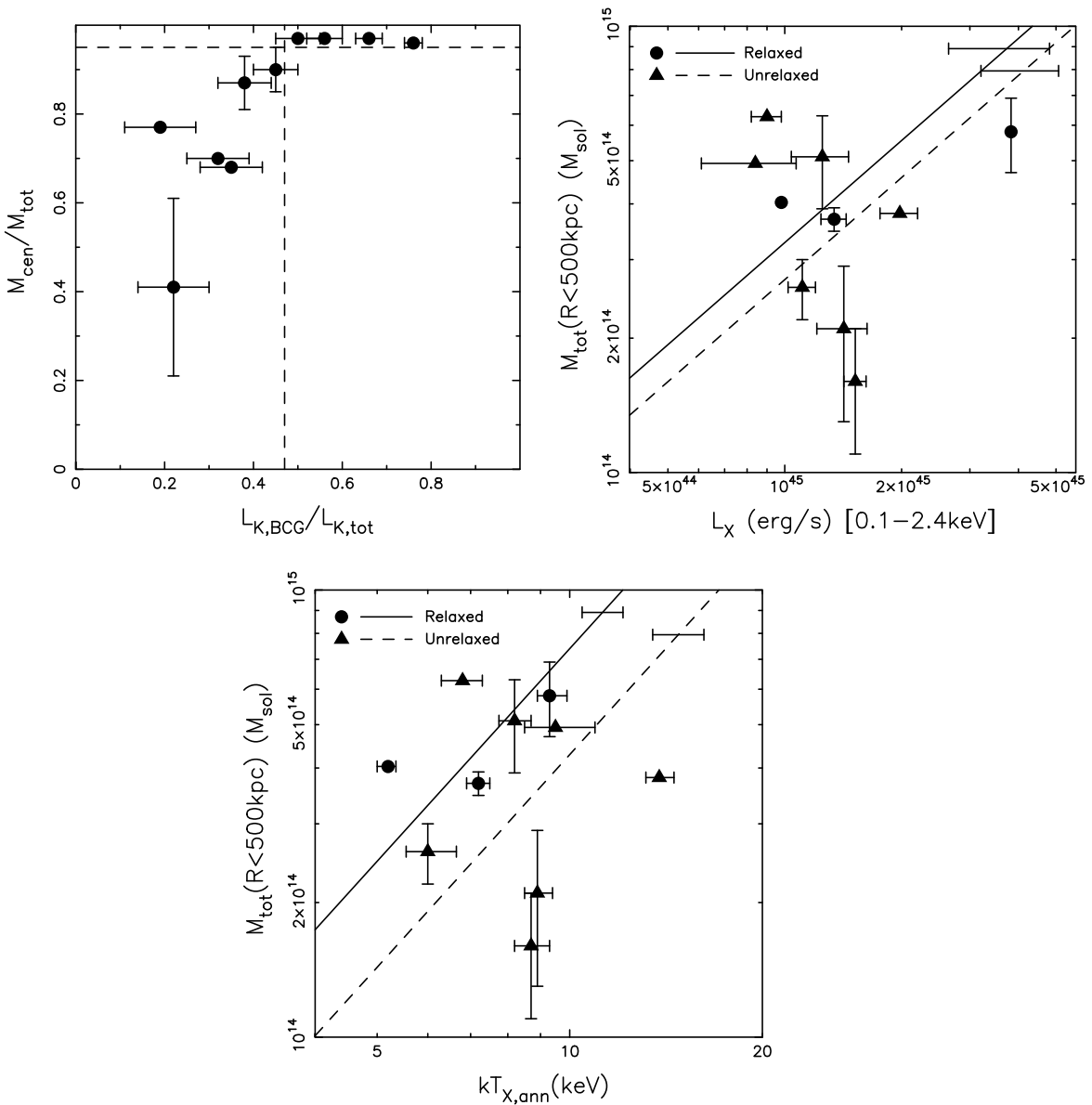

Fig. 27 Left panel: Central mass fraction (a measure of the dominance of the central dark matter halo), $M_{\text {cen }} / M_{\text {tot }}$ versus central K-band luminosity fraction (measures the dominance of the central galaxy), $L_{\mathrm{K}, \mathrm{BCG}} / L_{\mathrm{K}, \text { tot }}$. There is a remarkably clean separation between a homogeneous population of centrally concentrated clusters $\left(M_{\mathrm{cen}} / M_{\mathrm{tot}}>0.95, L_{\mathrm{K}, \mathrm{BCG}} / L_{\mathrm{K} \text {, tot }}>\sim 0.55\right)$ and a much more diverse population of less concentrated clusters. Center \& right panels: Mass $-L_{\mathrm{X}}$ and Mass- $-T_{\mathrm{X}}$ relations. The solid and dashed lines show the best-fit relations normalized by the relaxed and unrelaxed clusters, respectively. The error bars on each line show the uncertainty on the normalizations. The scatter in the Mass $-L_{X}$ relation appears to be symmetric; in the mass- $T_{X}$ relation the normalization of the unrelaxed clusters appears to be $40 \%$ hotter than the relaxed clusters at $2 \sigma$ significance. Figures from Smith et al. (2005)

(CCCP) that will add 30 more X-ray selected clusters observed with the CFHT12k or Megacam camera to the initial set of 20 clusters. Lensing results are, however, still pending at the time of writing this review.

\subsection{On-going and future cluster lensing surveys}

Clusters of galaxies are complicated systems that are rapidly assembling and evolving, nevertheless they are considered to be very good tracers of the underlying cosmology (and in particular could probe Dark Energy) as well as a way to measure the 

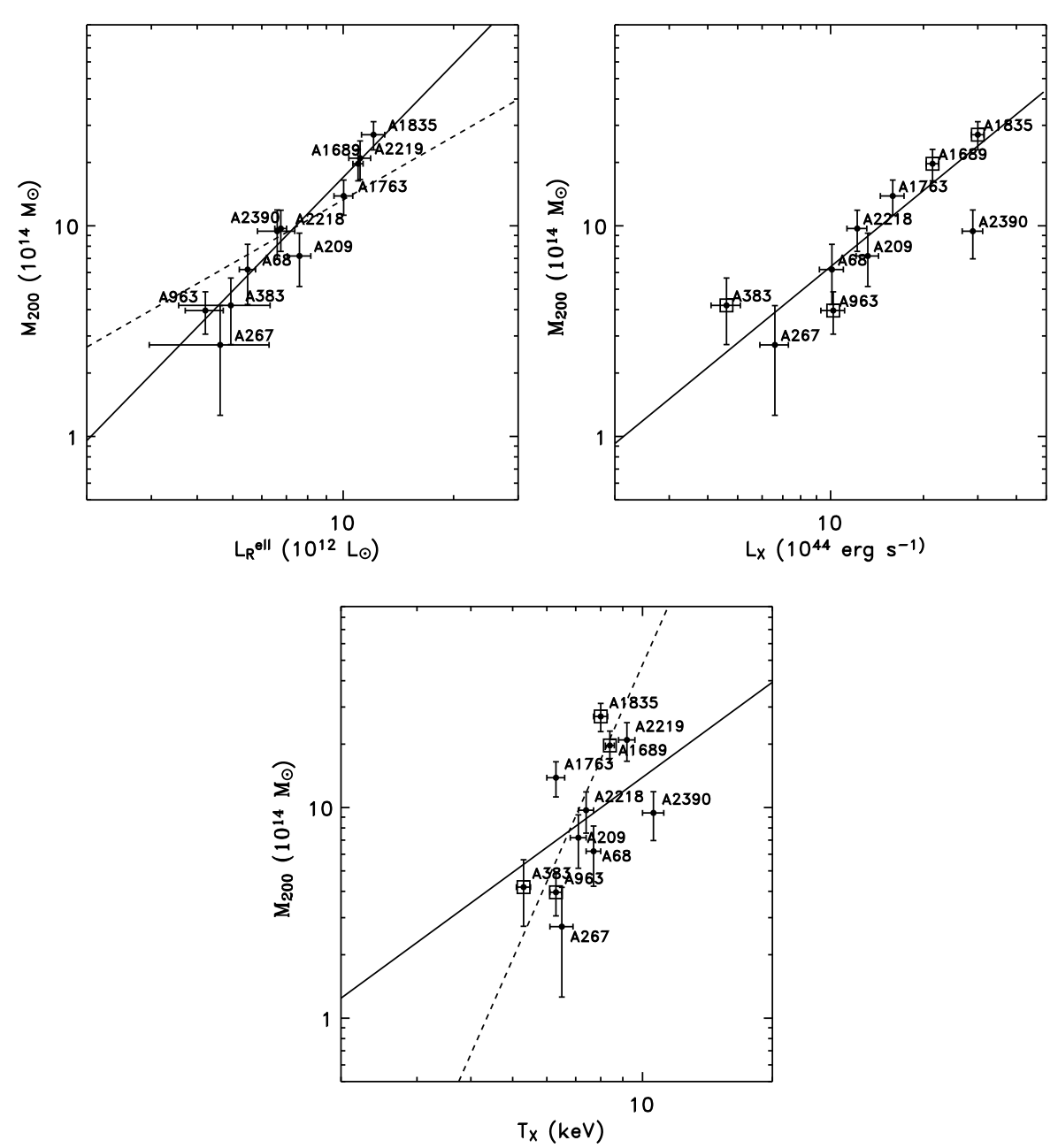

Fig. 28 Left panel: Lensing 2D mass versus optical luminosity for the clusters in the Bardeau et al. (2007) sample (12 X-ray bright clusters selected to be at $z \sim 0.2$ ). The lensing mass is computed at the virial radius $r_{200}$ derived from the best weak lensing fits. The luminosity is computed in the $R$ band for the cluster red sequence galaxies. Dashed line represents a constant $M / L$ ratio of 133 in solar units. The solid line is the best-fit power law $M \propto L^{1.8}$. Center panel: Weak lensing 3D virial mass $M_{200}$ versus X-ray luminosity. The best-fit line has a slope $\alpha=1.20 \pm 0.16$. Right panel: Weak lensing 3D virial mass $M_{200}$ versus X-ray temperature. The straight line corresponds to a $M_{200} \propto T^{3 / 2}$ relation while the dashed line corresponds to the best-fit power law relation $M \propto T^{4.6 \pm 0.7}$. Temperatures are derived from XMM data (Zhang et al. 2007), including A 2219 from ASCA data (Ota et al. 2004). The 4 clusters with cooling core or relaxed properties are marked with empty boxes. (From Bardeau et al. 2007)

growth of structure, thus potentially sensitive to gravity and to the nature of Dark Matter. A better understanding of clusters will be possible only with larger cluster samples, as earlier work and conclusions therefrom were limited by statistics. The number of massive clusters with published lensing data is steadily growing, as is the number of cosmological surveys in which clusters can be studied with strong and 
weak lensing techniques, either directly from the survey data or by further followups.

Four techniques are avidly pursued to search for clusters:

- Photometric searches that use wide field imaging surveys such as the Sloan Digital Sky Survey (SDSS), the Red-sequence Cluster Survey (RCS), and the CFHT Legacy Survey (CFHTLS). Furthermore, new photometric surveys have just started or will start in the next year, namely the VST KIDS survey, the Dark Energy Survey (DES), and the Subaru Hyper-Suprime Camera (HSC) survey.

- X-ray selected cluster searches: (i) based on the follow-up of the ROSAT All Sky Survey: such as the MAssive Cluster Survey (MACS) (Ebeling et al. 2001) and the REFLEX survey (Boehringer et al. 2004) (ii) based on dedicated (or serendipitous) X-ray ROSAT or XMM imaging surveys such as: WARPS (Scharf et al. 1997), SHARC (Collins et al. 1997), the ROSAT Deep Cluster Survey (Rosati et al. 2001), XDCS (the XMM Deep Cluster Survey, see Fassbender et al. 2008), XCS (Romer 2008); and XMM-LSS (Pierre et al. 2007).

- SZ searches: e.g. Atacama Cosmology Telescope Cluster Survey (Hincks et al. 2010; Marriage et al. 2011; Hand et al. 2011), the South Pole Telescope Cluster Survey (Chang et al. 2009; Vanderlinde et al. 2010; Plagge et al. 2010) and the Planck mission (Ade et al. 2011).

- Weak and Strong lensing searches based on photometric surveys, or following up $\mathrm{X}$-ray or SZ selected clusters.

We focus on the latter techniques in the following sub-sections.

\subsection{Targeted cluster surveys}

\subsubsection{The Local Cluster Substructure Survey (LoCuSS)}

LoCuSS extends Smith et al.'s (2005) pilot study of 10 X-ray luminous clusters at $z=0.2$ to an order of magnitude larger sample at $0.15<z<0.3$, drawn from the ROSAT All-sky Survey Catalogues (Ebeling et al. 1998, 2000, 2004). The main lensing-related goals of LoCuSS are to measure the mass, internal structure, and thermodynamics of a complete volume-limited sample of 80 clusters observable from Mauna Kea, and thus to obtain definitive results on the mass-observable scaling relations at low redshift. The normalization, shape, scatter (and any structural segregation detected) of these scaling relations will calibrate the properties of low-redshift clusters as an input to cluster-based cosmology experiments, and to help interpret high-redshift cluster samples.

To date LoCuSS has published weak lensing analysis of 30 clusters observed with Suprime-CAM on the Subaru 8.2-m telescope (Okabe et al. 2010; see also Oguri et al. 2010). The main results from this statistical study are that (i) a simple colormagnitude selection of background galaxies yields samples that are statistically consistent with negligible residual contamination by faint cluster members, albeit with large uncertainties, (ii) cluster density profiles are curved (in log-log space), and statistically compatible with the Navarro et al. (1997) profile, and (iii) based on the NFW profile model fits, the normalization of the mass-concentration relation of X-ray selected clusters is consistent with theoretical $\Lambda$ CDM-based predictions, although the 
slope of the observed relation may be steeper than predicted. The last of these results is particularly interesting in the context of detailed studies of individual clusters selected to have a large Einstein radius. As noted in Sect. 7, such objects are often found to have concentrations that exceed the CDM prediction by factors of 2-3 (Comerford and Natarajan 2007; Oguri et al. 2009). Okabe et al.'s results from 30 X-ray-selected clusters indicate that the large Einstein radius selection in earlier work introduces a strong bias.

Comparison of Okabe et al.'s weak lensing mass measurements with X-ray and Sunyaev-Zel'dovich (SZ) effect probes has so far been limited by the presence of outlier clusters in the small samples for which the relevant data are available. For example, the well-known merging cluster A1914 strongly influences the results in the X-ray/lensing comparison of 12 clusters for which Subaru and XMM-Newton data are available (Okabe et al. 2010; Zhang et al. 2010). More recently, Marrone et al. (2011) presented the first weak-lensing-based mass-SZ scaling relation based on Subaru and Sunyaev-Zel'dovich Array (SZA) observations of 18 clusters. Encouragingly, this relation is consistent with self-similar predictions, although it presents $20 \%$ scatter in natural $\log$ of mass at fixed integrated $Y$-parameter-a factor of 2 more scatter than found in studies that use X-ray data and assume hydrostatic equilibrium to infer cluster mass. Indeed, the normalization of the $M_{\mathrm{WL}}-Y$ relation at $\Delta=500$ (roughly $1 \mathrm{Mpc}$ ) for undisturbed clusters is $40 \%$ higher in mass than that for disturbed clusters. Marrone et al. identified several of the undisturbed clusters as likely prolate spheroids whose major axis is closely aligned with the line of sight as being largely responsible for this segregation. These results highlight the feasibility and growing maturity of lensing-based studies of large cluster samples, and also emphasize that much important work remains to be done to fully understand the optimal methods for cluster mass measurement.

\subsubsection{The MAssive Cluster Survey}

The MAssive Cluster Survey (MACS) is an on-going project aimed at the compilation and characterization of a statistically complete sample of very X-ray luminous (and thus, by inference, massive), distant clusters of galaxies. The primary goal of MACS was to increase the number of known massive clusters at $z>0.3$ from a handful to a hundred. To achieve these goals, Ebeling et al. (2001) applied an X-ray flux and X-ray hardness-ratio cut to select distant cluster candidates from the ROSAT Bright Source catalog. Starting from a list of more than $5000 \mathrm{X}$-ray sources within the survey area of 22,735 square degrees they use positional cross-correlations with public catalogs of Galactic and extragalactic objects, with reference to APM colors, visual inspection of Digitized Sky Survey images, extensive CCD imaging, and finally spectroscopic observations with the University of Hawaii's 2.2-m and the Keck 10 -m telescopes to compile the final cluster sample. The MACS cluster sample comprises 124 spectroscopically confirmed clusters at $0.3<z<0.7$ (Fig. 29). Comprehensive follow-up observations of MACS clusters include: weak lensing mass measurements using wide-field SUBARU imaging data, virial mass estimates based on cluster galaxy velocity dispersions measured with the CFHT and Keck, SZ observations with the BIMA mm-wave radio interferometer, measurements of the cluster gas and temperature distribution with Chandra, and both deep, multi-passband 


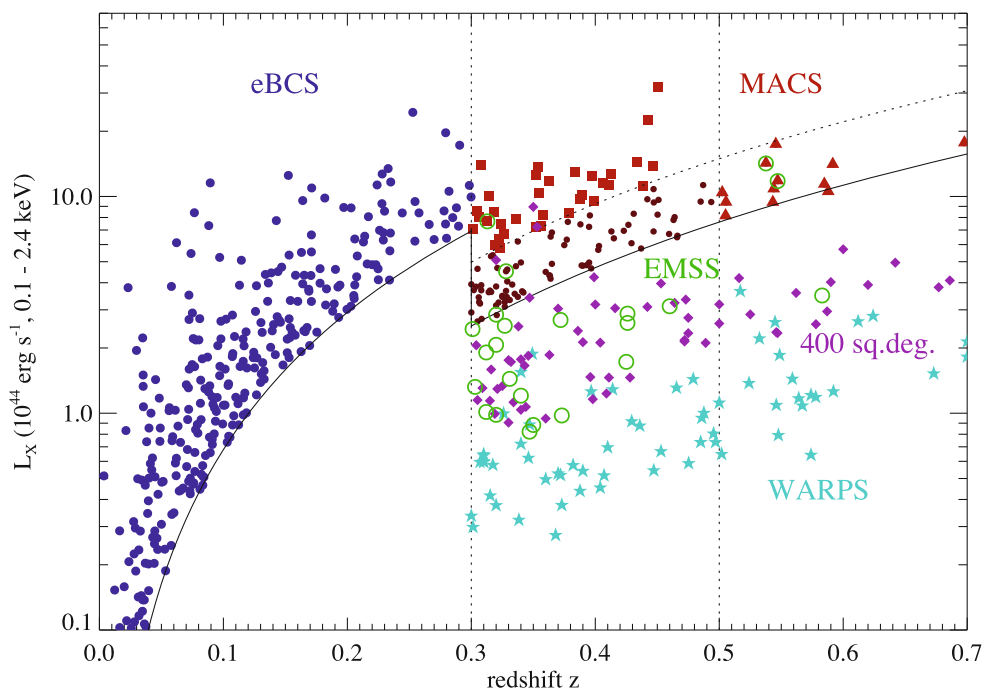

Fig. 29 The luminosity versus redshift plot comparing the MACS surveys to a number of other X-ray surveys: EMSS, eBCS, WARPS, the 400 square degree survey. It is evident from this figure that MACS is very efficient in selecting the most massive X-ray clusters at $z>0.3$

and snapshot images with HST. A large number of MACS clusters are strong lenses and some of them have been studied in detail: MACS J1206-0847 (Ebeling et al. 2009); MACS J1149.5+2223 (Smith et al. 2009); MACS J1423.8+2404 (Limousin et al. 2010; Morandi et al. 2010). MACS J0025.4-1222 (Bradač et al. 2008) was identified as a merging cluster with some similarity to the "Bullet Cluster". Zitrin et al. (2011a) presented the results of a strong lensing analysis of the complete sample of the 12 MACS clusters at $z>0.5$ using HST images. The distribution of Einstein radii has a median value of $\sim 28$ arcseconds (for a source redshift of $z_{\mathrm{S}} \sim 2$ ), twice as large as other lower-z samples, making the MACS sample a truly massive cluster sample confirmed by the numerous strong lensing discoveries. One of the most extreme clusters known presently is likely MACS J0717.5+3745 (Ebeling et al. 2004) which was recognized as a complex merger of four individual substructures, with a long tailed filamentary structure. The four sub-structures have all been identified in a recent lensing mass reconstruction by Limousin et al. (2011) and the filamentary structure was directly measured by weak lensing measurements with a 18-pointing HST mosaic (Jauzac et al. 2011). Horesh et al. (2010), investigated the statistics of strong lensed arcs in the X-ray selected MACS clusters versus the optically selected RCS clusters (see below). They measured the lensed-arc statistics of 97 clusters imaged with HST, identifying lensed arcs using two automated arc-detection algorithms. They compile a catalog of 42 arcs in MACS and seven arcs in the RCS. At $0.3<z<0.7$, MACS clusters have a significantly higher mean frequency of arcs, $1.2 \pm 0.2$ per cluster, versus $0.2 \pm 0.1$ in RCS, which can easily be explained by the nature of the selection of these two different cluster samples. 


\subsubsection{ESO distant cluster survey}

Nevertheless, optical selection is still common specially for high-redshift clusters $(z>0.6)$ where X-ray selection is limited. A particular focused and productive survey is the ESO distant cluster survey (EDiSC, White et al. 2005). EDiSC is a survey of 20 fields containing distant galaxy clusters $(0.4<z<1.0)$ chosen amongst the brightest objects identified in the Las Campanas Distant Cluster Survey. They were confirmed by identifying red sequences in moderately deep two color data from VLT/FORS2, and further investigations with VLT in spectroscopy, the ESO Wide Field Imager, and HST/ACS mosaic images for 10 of the most distant clusters. Using the deep VLT/FORS2 data, Clowe et al. (2006) measured the masses for the EDiSC clusters. In particular, they compared the mass measurements of 13 of the EDiSC clusters with luminosity measurements from cluster galaxies selected using photometric redshifts and find evidence of a dependence of the cluster mass-to-light ratio with redshift.

\subsubsection{Red-sequence cluster surveys}

Another important optically selected cluster survey is the $100 \mathrm{deg}^{2}$ Red-Sequence Cluster Survey (RCS, Gladders 2002, Gladders and Yee 2005) and its $1000 \mathrm{deg}^{2}$ RCS-2 extension (Gilbank et al. 2011), which are based on shallow multi-color imaging with the CFHT12k and Megacam cameras. RCS-2 covers $\sim 1000 \mathrm{deg}^{2}$ and includes the first RCS area, it reaches $5 \sigma$ point-source limiting magnitudes in $[g, r, i, z]=[24.4,24.3,23.7,22.8]$, approximately 1-2 magnitudes deeper than the SDSS. RCS-2 is designed to detect clusters over the redshift range $0.1<z<1$, building a statistically complete, large $\left(\sim 10^{4}\right)$ sample of clusters, covering a sufficiently long redshift baseline to be able to place constraints on cosmological parameters probed via the evolution of the cluster mass function. Furthermore, a large sample of strongly lensed arcs associated with these clusters has been derived (e.g. Gladders et al. 2002, 2003), and weak lensing measurements from the most massive clusters detected in RCS-2 is likely possible.

\subsubsection{The Multi-Cluster Treasury: CLASH survey}

The recently approved MCT (Multi-Cluster Treasury) program on HST will achieve multi-band imaging of a sample of 25 X-ray selected clusters (Postman et al. 2011), thus providing detailed photometric-redshift estimates for multiple images. This sample with appropriate ground based follow-up is likely to provide important insights into many of the current unsolved problems in cluster assembly and evolution. Dedicated lensing studies will enable detailed investigation of their mass distributions (Zitrin et al. 2011b, 2011c) and will help find some efficient lenses that can be exploited to study the distant Universe by using them as gravitational telescopes (Richard et al. 2011) — a topic that will be discussed further in the next section.

\subsection{Cluster lenses in wide cosmological surveys}

The previous sub-section focused on targeted cluster surveys. However, cluster lenses can also be found in wide cosmological surveys (e.g. Wittman et al. 2001, 2003; Hamana et al. 2004; Maturi et al. 2005). We briefly outline some of the most representative surveys of this decade starting from the widest to the deepest. 


\subsubsection{The Sloan Digital Sky Survey}

The Sloan Digital Sky Survey (SDSS) is an imaging and spectroscopic survey covering 10,000 $\mathrm{deg}^{2}$ (Aihara et al. 2011). Although this survey was not designed or optimized to measure cluster lensing, interesting results have been produced from detected strong and weak lensing measurements of clusters. Estrada et al. (2007) investigated the Sloan images of 825 SDSS galaxy clusters searching for giant arcs. Both a visual inspection of the images and an automated search were performed, and no arcs were found. They nevertheless report a serendipitous discovery of a bright arc in the Sloan images of an as yet unknown cluster. Hennawi et al. (2008) presented the first results of a strong lensing imaging survey (using the WIYN and UH $2 \mathrm{~m}$ telescope) targeting the richest clusters (with $0.1<z<0.6$ ) selected from SDSS. From a total of 240 clusters followed-up, they uncovered 16 new lensing clusters with definite giant arcs, 12 systems for which the lensing interpretation is very likely, and 9 possible lenses which contain shorter arclets or candidate arcs which require further observations to confirm their lensing origin. The new lenses discovered in this survey will enable future systematic studies of the statistics of strong lensing and their implications for cosmology and the current structure formation paradigm. Kubo et al. (2009) and then Diehl et al. (2009) identified 10 strongly lensed galaxies as part of the "Sloan Bright Arcs Survey". Follow-up imaging identified the lensing systems as group-scale lenses, an intermediate regime between isolated galaxies and galaxy clusters (see Cabanac et al. 2007). Bayliss et al. (2011) presented the results from a spectroscopic program targeting 26 strong lensing clusters $(0.2<z<0.65)$ visually identified in SDSS or RCS-2 revealing 69 unique background sources with redshifts as high as $z=5.2$, which will enable robust strong lensing mass models to be constructed for these clusters (some of the most remarkable clusters discovered are presented in Fig. 30).

On the weak lensing side, the first measurements were conducted by Sheldon et al. (2001). Later on Rykoff et al. (2008) measured the scaling relation between X-ray luminosity and the total mass for 17,000 galaxy clusters in the SDSS maxBCG cluster sample. To achieve this, they stacked sub-samples of clusters within fixed ranges of optical richness, and they measured the mean X-ray luminosity $L_{\mathrm{X}}$, and the weak lensing mean mass, $\left\langle M_{200}\right\rangle$. For rich clusters, they found a power law correlation between $L_{\mathrm{X}}$ and $M_{200}$ with a slope compatible with previous estimates based on Xray selected catalogs. Furthermore, Rozo et al. (2010) used the abundance and weak lensing mass measurements of the SDSS maxBCG cluster catalog to simultaneously constrain cosmology and the cluster richness-mass relation. Assuming a flat $\Lambda \mathrm{CDM}$ cosmology, they found that $\sigma_{8}\left(\Omega_{\mathrm{m}} / 0.25\right)^{0.41}=0.832 \pm 0.033$. These constraints are fully consistent with those derived from WMAP five-year data. With this remarkable consistency they claim that optically selected cluster samples may produce precision constraints on cosmological parameters in future wide-field imaging cosmological surveys.

\subsubsection{The CFHT-legacy survey}

Soon after the first light of the Megacam camera at CFHT, a legacy survey (LS) was started. It comprises a deep $u, g, r, i, z(i \sim 27.5)$ survey of 4 square degrees in four 

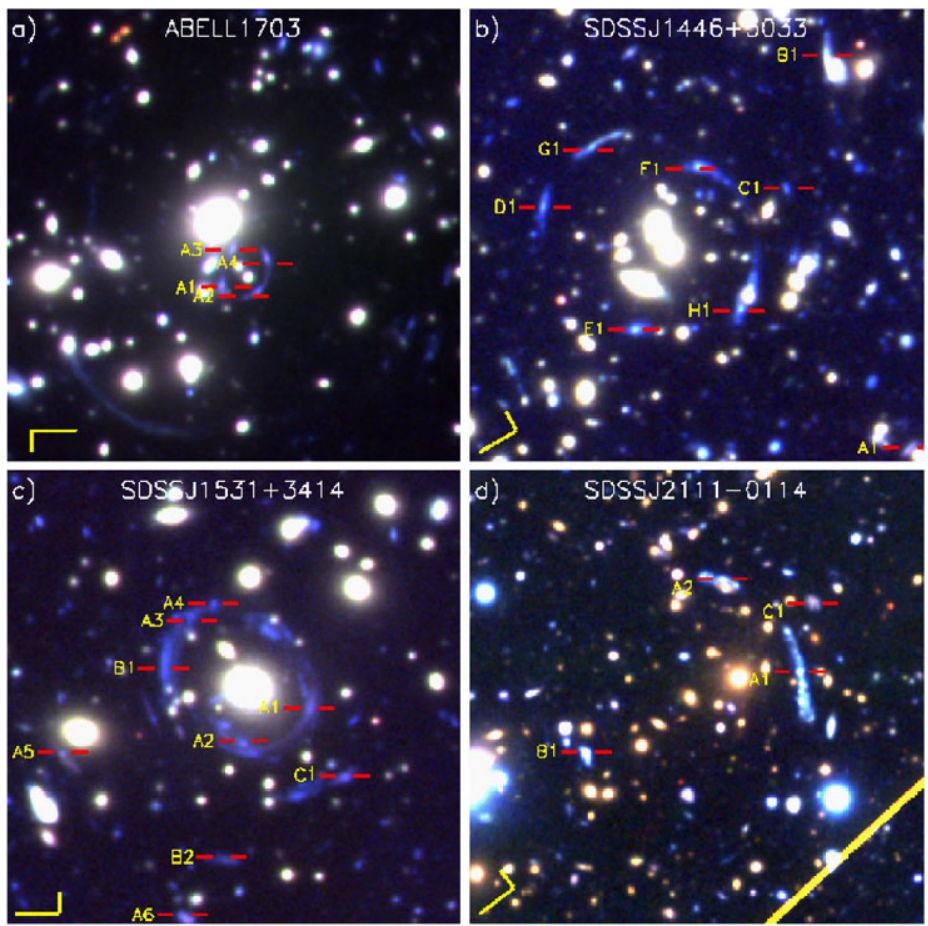

Fig. 30 SDSS discovered strong lensing clusters-(a) Abell 1703, (b) SDSS J1446+3033, (c) SDSS $\mathrm{J} 1531+3414$, and (d) SDSS J2111-0114. Color composite images are made from $g, r, i$ imaging obtained with Subaru/SuprimeCam. All images are $75^{\prime \prime} \times 75^{\prime \prime}$. Background sources are bracketed by red lines and labeled. Source labels with the same letter but different numbers (e.g. A1, A2, etc.) have the same redshifts to within the measurement errors, and are presumed to be the same source, multiply imaged (figure from Bayliss et al. 2011)

independent fields spread across the sky, and a wide synoptic $u, g, r, i, z(i \sim 24.5)$ survey of 170 square degrees in four patches of 25 to 72 square degrees. Due to the excellent seeing delivered by CFHT, the Legacy Survey has lead to intensive strong and weak lensing studies.

In particular, Cabanac et al. (2007) have searched for strong lensing arcs and Einstein rings around galaxies in both the deep and wide part of the CFHT-LS. Most of the systems uncovered have deflection angles ranging between 2 and 15 arcseconds. Such samples have thus uncovered a large population of strong lenses from galaxy groups with typical halo masses of about $10^{13} h^{-1} \mathrm{M}_{\odot}$. The 13 most massive systems have been studied in detail by Limousin et al. (2009), and detailed analysis of the mass distribution on small and large scales has been investigated by Suyu and Halkola (2010) and Limousin et al. (2010), respectively. A weak lensing search for galaxy clusters in the 4 square degrees of the 4 CFHT-LS deep fields was performed and results are presented in Gavazzi and Soucail (2007). Using deep $i$-band images they performed weak lensing mass reconstructions and identified high convergence peaks. They used galaxy photometric redshifts to improve the weak lensing analysis. Among the 14 peaks found above $3.5 \sigma$, nine were considered as secure detections 
upon cross-correlation studies with optical and X-ray catalogs. Berge et al. (2008) conducted a joint weak lensing and X-ray analysis of (only) 4 square degrees from the CFHTLS and XMM-LSS surveys. They identified six weak-lensing-detected clusters of galaxies, and showed that their counts can be used to constrain the power-spectrum normalization $\sigma_{8}=0.92_{-0.30}^{+0.26}$ for $\Omega_{\mathrm{m}}=0.24$. They showed that deep surveys should be dedicated to the study of the physics of clusters and groups of galaxies, and wide surveys are preferred for the measurement of cosmological parameters. A first catalogue of lensing selected cluster has been recently published by Shan et al. (2011) on the CFHT-LS W1 field. They perform a weak lensing mass map reconstruction and identify high signal-to-noise ratio convergence peaks, which were then correlated with the optically selected cluster catalogue of Thanjavur et al. (2011). They then used tomographic techniques to validate their most significant detections and estimate a tomographic redshift. More weak lensing cluster analyses are expected to be published from CFHT-LS in the near future.

\subsubsection{The COSMOS survey}

With only 2 square degrees the COSMOS Survey focused on the relatively highredshift Universe. Due to the relatively small volume probed, COSMOS is unlikely to find the most massive structures in the Universe, but it has delivered interesting constraints on the redshift evolution of clusters and the scaling relations between observables. Thanks to the deep X-ray observation of COSMOS fields, clusters can be efficiently selected in principle out to $z \sim 2$. Taking advantage of the X-ray selected catalog, Leauthaud et al. (2010) have investigated the scaling relation between X-ray luminosity $\left(L_{X}\right)$ and the weak lensing halo mass $\left(M_{200}\right)$ for about $200 \mathrm{X}$ ray-selected galaxy groups. Weak lensing profiles and halo masses were derived for nine sub-samples, narrowly binned in luminosity and redshift. The COSMOS data alone are well fit by a power law, $M_{200} \propto L_{\mathrm{X}}^{\alpha}$, with a slope $\alpha=0.66 \pm 0.14$. These observations significantly extend the dynamic range for which the halo masses of $\mathrm{X}$-ray-selected structures have been measured with weak gravitational lensing as shown in Fig. 31. Combining with other measurements demonstrates that the $M-$ $L_{\mathrm{X}}$ relation is well described by a single power law with $\alpha=0.64 \pm 0.03$, over two decades in mass: $M_{200} \sim 10^{13.5}-10^{15.5} h_{72}^{-1} \mathrm{M}_{\odot}$. These results confirm that clusters do not follow the self-similar evolution model with $\alpha=0.75$ proposed by Kaiser (1986).

\section{Cluster lenses as nature's telescopes}

\subsection{Magnification due to gravitational lensing}

Cluster lenses magnify and distort the shapes of distant galaxies that lie behind them. For strong lensing clusters, the amplification factor can in principle be infinite if the source is compact enough and is located exactly behind the caustic, of course such an event is infinitely rare! Nevertheless, in several strong lensing clusters, amplification factors larger than $40 \times(\sim 4$ magnitudes) have been measured (Seitz et al. 1998), and 


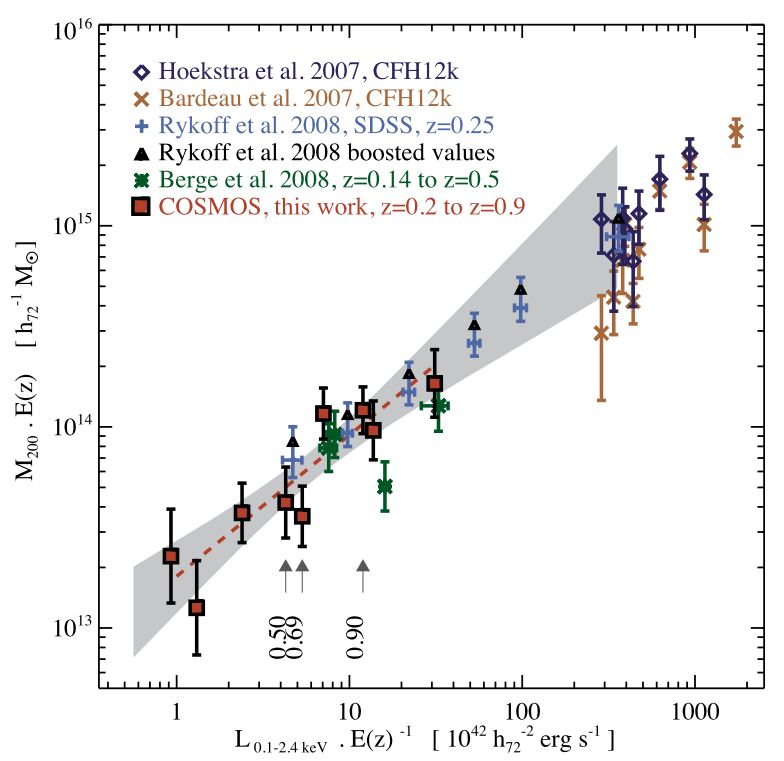

Fig. 31 The COSMOS $M-L_{X}$ relation from Leauthaud et al. (2010). Dark blue diamonds show individually detected clusters from Hoekstra et al. (2006) with updated masses from Madhavi et al. (2008). Sienna cross symbols show data points from Bardeau et al. (2007). Light blue plus symbols represent the Rykoff et al. (2008) results from a stacked analysis in the SDSS and black diamonds take into account a recent correction to these masses due to a new calibration of the source distribution. The upper error bars have been adjusted to account for the redshift uncertainty. Green asterisks show four data points at intermediate masses from Berge et al. (2008). Finally, the red squares depict our COSMOS results which extend previous results to lower masses and to higher redshifts. Three arrows highlight the highest redshift COSMOS data points. The grey shaded region shows the upper and lower envelope of the ensemble of lines with a slope and intercept that lie within the 68 percent confidence region

in fact, amplification factors larger than $4 \times(\sim 1.5$ magnitudes $)$ are quite common (Richard et al. 2011). It is thus not surprising that "Cluster Lenses" are often referred to as "Nature's Telescopes" or "Cosmic Telescopes" and have been rather effectively used to discover and study the most distant galaxies that lie behind them.

The regions with the largest magnification are the regions closest to the critical lines in the image plane (typically less than 1 square arcminute), and closest to the caustic line in the source plane (typically a few tens to hundreds square arcsecond). The cross section for high amplification will vary from cluster to cluster and depends on the detailed mass distribution. To first order, the cross-section scales with the square of the Einstein radius as well as with the ellipticity or anisotropy of the projected mass distribution on the sky.

As the magnification is wavelength independent, the benefit of using cluster lenses as cosmic telescopes has been exploited at various wavelengths, from X-ray to the radio domain. Lensing clusters were first used as cosmic telescopes in the opti$\mathrm{cal} / \mathrm{NIR}$ domain, where a large population of the most distant galaxies (at their time of discovery) were found behind these cluster magnified regions (e.g. Yee et al. 1996; Franx et al. 1997; Ellis et al. 2001; Hu et al. 2002; Kneib et al. 2004; Richard et al. 2011). Lensing clusters were also used at longer wavelengths in sub- 
millimeter using SCUBA at JCMT (e.g. Smail et al. 1998) and in the mid-infrared domain using the ESA ISO space telescope (Altieri et al. 1999; Metcalfe et al. 2003; Coia et al. 2005a, 2005b) and now in the far-infrared using the Herschel space observatory (Egami et al. 2010; Altieri et al. 2010).

There are two important and unprecedented advantages that cluster lenses offer as cosmic telescopes as they provide the largest field of view with high magnification:

- the potential discovery of the most distant objects and low-luminosity objects that would otherwise remain undetected with similar blank field imaging,

- the possibility to study the morphology of distant galaxies which otherwise would not be resolved and explore their physical properties that would otherwise be impossible to characterize.

Furthermore, we note that as cluster lenses magnify they also distort the shapes of distant galaxies. In general, the further the sources, the stronger the distortions. Hence to first order, the shape of a lensed galaxy (assuming it can be resolved), and whether it is multiply imaged or not, can be used as a good distance indicator.

\subsection{Cosmic telescope surveys}

Rather similar to other galaxy surveys two distinct observational strategies that tradeoff depth with area have been explored thus far:

(1) deep mapping (in imaging or spectroscopy) of a few well modeled lensing clusters to search for distant lensed sources - this allows us to probe down the luminosity function of the targeted distant source population,

(2) shallow mapping on a large cluster sample to search for rare highly magnified background sources (e.g. Fig. 32), with the idea to thereafter conduct detailed follow-up observations of these sources benefiting from the high amplification/magnification to constrain important physical and morphological properties of high-redshift sources (e.g. Lemoine-Busserolle et al. 2003).

As the strong magnification region covers typically at most about a few square arcminutes, surveys through cluster lenses are particularly adapted to those instruments/telescopes that have an instantaneous field of view comparable to the strong lensing region. The HST cameras are particularly well matched to cluster strong lensing regions and are thus very well adapted to use for the search and study of distant sources. Incidentally, this was also the case for the SCUBA instrument on JCMT, as well as the ISOCAM on the ISO space mission. In the near future ALMA, MUSE (the one square arcminute integral field unit on the VLT), and the forthcoming JWST instruments are all facilities that will most effectively exploit lensing magnification.

Of course when conducting a detailed follow-up study of highly magnified sources, the most effective instruments are high-resolution imagers and spectrographs. In particular, because of the extended nature of the most amplified sources, integral field spectrographs are more adapted compared to long-slit instruments, and it is thus natural to conduct follow-up studies with instruments such as SINFONI on VLT or OSIRIS on Keck for the extremely magnified, rare, lensed sources.

Finally, an other particular observation strategy of cosmic telescope is that of "critical line mapping". In this case, one specifically targets regions near the critical lines 


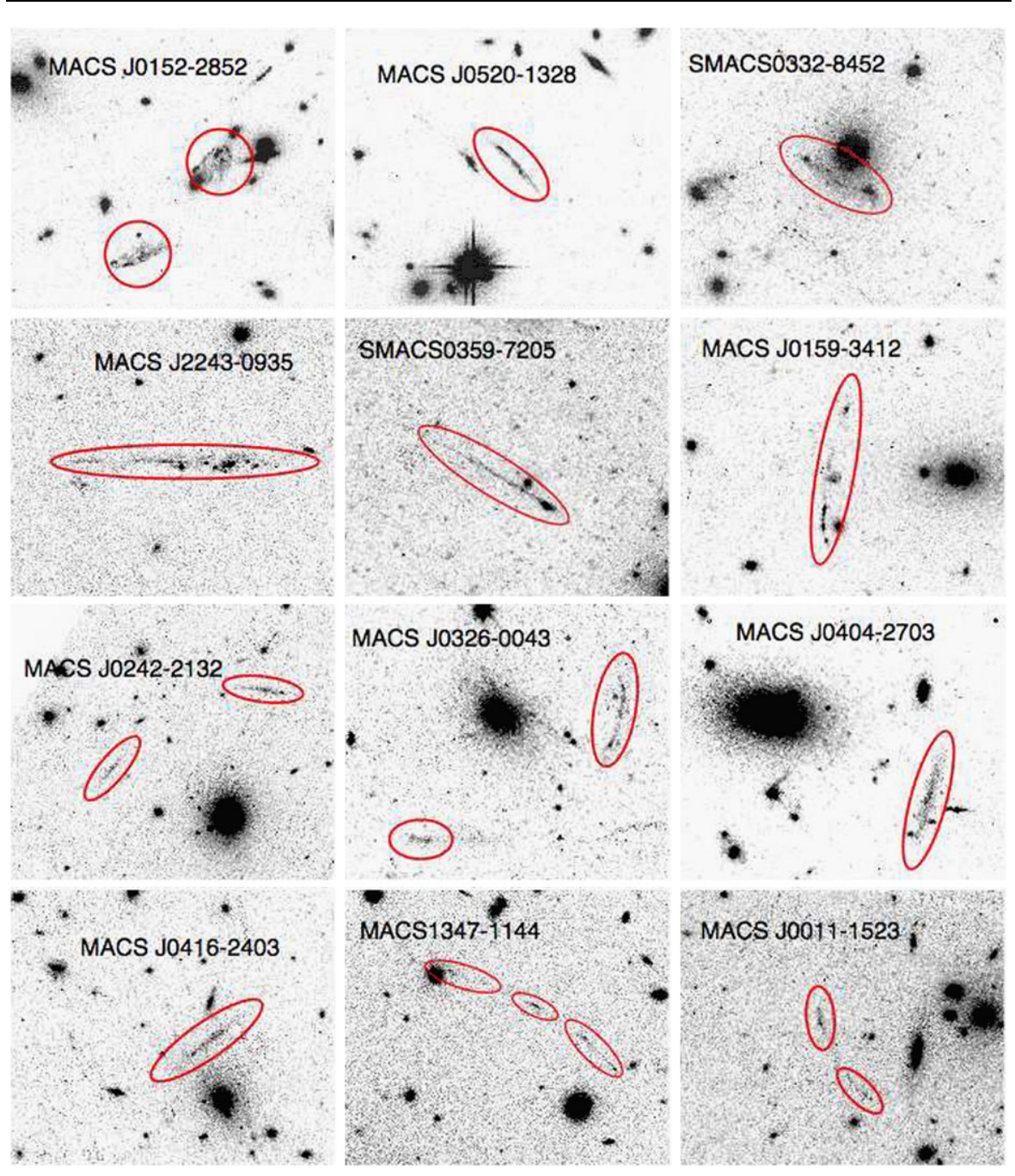

Fig. 32 Newly discovered giant arcs or multiple images as part at the SNAPSHOT HST observations (PI. H. Ebeling) of the MACS cluster sample. These images show the diversity of morphology for these serendipitously discovered strongly lensed galaxies (figure is from Richard et al. private communication)

using dedicated instruments such as a long-slit spectrograph (e.g. Santos et al. 2004; Stark et al. 2007), an integral field spectrograph (Clement et al. 2011) or a millimeter wave interferometer to blindly probe the distant Universe. In this case the effective field of view of the instrument is small compared to the critical region, thus requiring a mapping strategy to cover the region with the highest amplification.

\section{3 "Lens redshift" measurement}

As lensing distortion and magnification are a function of the redshift of the background sources, once a cluster mass distribution is well known, the lens model can 
be used to predict the redshifts for the newly identified multiple systems (e.g. Kneib et al. 1993; Natarajan et al. 1998; Ellis et al. 2001) as well as for the arclets (Kneib et al. 1994, 1996).

For multiple-image systems, the relative positions of the different images is a strong function of the redshift of the background source. Although the redshift sensitivity decreases with the redshift of the background source (because the $D_{\mathrm{LS}} / D_{\mathrm{S}}$ variation is smaller with increasing redshift) it can nevertheless be used to easily distinguish between low- and high-redshift solution. In this respect, one can easily discriminate $z \sim 1-2$ obscured galaxies from $z>4$ high-redshift lensed galaxies, and this property has been used many times very effectively (Ellis et al. 2001; Kneib et al. 2004; Richard et al. 2008, 2011). For the arclets, the redshift prediction is based on the fact that on average a distant galaxy is randomly orientated, and its ellipticity follows a relatively peaked ellipticity distribution $\left(\sigma_{\epsilon} \sim 0.25\right)$. Hence, by conducting high-resolution imaging (e.g. with Hubble), and by measuring the ellipticity and orientation of the background lensed sources in the core of massive cluster lenses, one can statistically derive the redshift distribution of the background lensed population. Such measurements were first introduced by Kneib et al. (1993), and developed further in Kneib et al. (1996). These predictions were tested and verified by Ebbels et al. (1998) using spectroscopy in the case of the lensing cluster Abell 2218. Despite the successful demonstration of the technique, it never became popular due to the following limitations. First, the derived probability distribution $p(z)$ distribution is relatively broad, particularly, at high redshift. Therefore the method is not really competitive with photometric-redshift determinations, except may be for disentangling catastrophic photometric redshifts. Second, the cluster galaxy contamination is high in the optical/near-infrared domain and statistical estimates always have limited utility. Third, galaxy sizes decrease rapidly with redshift, and accurate measurement of the galaxy shape can only be done efficiently with deep Hubble imaging yet again limiting the use of this technique. Finally, as there are many other good science drivers to obtain multi-band information on these massive clusters, and as photometric-redshift determination methods are rapidly improving, the statistical lens redshift measurements never became attractive and/or popular.

\subsection{Lensing surveys in the sub-millimeter}

The SCUBA (the JCMT Submillimeter Common-User Bolometer Array) Lensing survey was likely one of the first systematic surveys to exploit distant lensed galaxies using massive clusters. This survey first started with the mapping of two massive clusters: Abell 370 at $z=0.37$ and $\mathrm{Cl} 2244-02$ at $z=0.33$ (Smail et al. 1997) and continued to map the region behind five similarly massive clusters covering a total area of 0.01 square degree (Smail et al. 1998). Each SCUBA continuum map from this cluster lens survey covered a total area of about 5 square arcminutes to $1 \sigma$ noise levels less than $14 \mathrm{mJy} /$ beam and $2 \mathrm{mJy} /$ beam at 450 and 850 micron wavelengths, respectively.

Since SCUBA was a new instrument that achieved a sensitivity 2-3 orders of magnitude deeper than was previously possible and thanks to the cluster magnification, Smail et al. (1997) were the first to find the distant sub-millimeter (sub-mm) 
selected galaxy population. In total 17 sources brighter than the $50 \%$ completeness limits (10 brighter than the $80 \%$ limit) were discovered (Smail et al. 1998). The submillimeter spectral properties of these first sources indicated that the majority lie at high redshift $(1<z<5.5)$, which was confirmed later with redshift measurements. Measured redshifts for a large number of these sub-mm-selected galaxies placed the bulk of this population at $z \sim 2.5$ (Ivison et al. 1998; Barger et al. 2002; Chapman et al. 2005).

The use of cluster lenses in the case of the sub-millimeter high-redshift searches was strongly motivated by the fact that cluster galaxy members are not sub-millimeter sources and are therefore transparent at this wavelength, making clusters perfect telescopes to preferentially probe the distant galaxy population (Blain 1997). Importantly, the use of cluster lenses increases the sensitivity of sub-mm maps and reduces the effects of source confusion (which plagues bolometer surveys in sub-mm and mm wavelengths) due to the dilution produced by lensing clusters. With accurate lens models Blain (1998) first corrected the observed sub-mm source counts for lensing amplification using the SCUBA lensing survey data on the first seven clusters, thus pushing the 850 micron counts down below the SCUBA confusion limit; for example at $1 \mathrm{mJy}, 7900 \pm 3000$ galaxies per square degree were found. Down to the $0.5 \mathrm{mJy}$ limit, the resolved 850 micron background radiation intensity was measured to be $(5 \pm 2) \times 10^{-10} \mathrm{~W} \mathrm{~m}^{-2} \mathrm{sr}^{-1}$, comparable to the current COBE estimate of the background, indicating for the first time that the bulk of the 850 micron background radiation is effectively produced by distant ultra-luminous galaxies. These first submm galaxy counts were confirmed later with a larger sample of clusters mapped by SCUBA (Cowie et al. 2002; Knudsen et al. 2008) reaching a lens-corrected flux limit of $0.1 \mathrm{mJy}$. The first sub-mm multiple images were found in Abell 2218 (Kneib et al. 2004) and MS0451-03 (Borys et al. 2004) identified at $z=2.516$ and $z \sim 2.9$, respectively. In particular, the source SMM J16359+6612 is gravitationally lensed by Abell 2218 into three discrete images with a total amplification factor of $\sim 45$, implying that this galaxy has an unlensed 850 -micron flux density of only $0.8 \mathrm{mJy}$. Furthermore, SMM J16359+6612 shows a complex morphology with three sub-components arguing for either a strong dust (lane) absorption or a merger. Interestingly, these sub-mm sources are surrounded by two other highly amplified galaxies at almost identical redshifts within a $\sim 100$-kpc region suggesting this sub-mm galaxy is located in a dense high-redshift group (see Fig. 33). Further mapping at the IRAM Plateau de Bure interferometer indicated that this source is a compact merger of two typical Lyman-break galaxies with a maximal separation between the two nuclei of about $3 \mathrm{kpc}$, thus it bears a close similarity to comparable luminosity, dusty starbursts that result from lower-mass mergers in the local Universe.

However, the most spectacular lensed sub-mm galaxy is certainly the recently discovered SMMJ2135-0102 at redshift $z=2.3259$ that is gravitationally magnified by a factor of 32 by the massive cluster MACSJ2135-010217 (Swinbank et al. 2010; Fig. 34). This large magnification, when combined with high-resolution sub-mm imaging, resolves the star-forming regions at a linear scale of just $\sim 100$ parsec. The luminosity densities of these star-forming regions are comparable to the dense cores of giant molecular clouds in the local Universe, but they are $\sim 100 \times$ larger and $10^{7}$ times more luminous. The star formation processes at $z \sim 2$ in this vigorously star- 

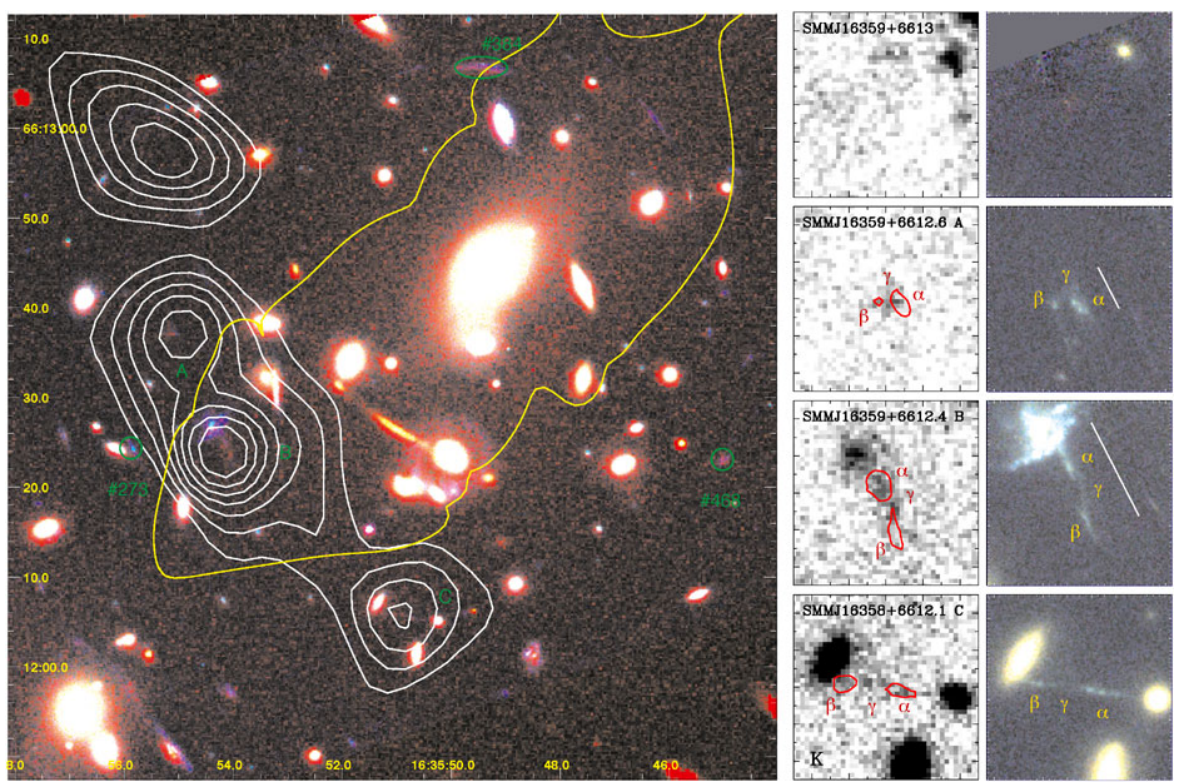

Fig. 33 (Left) A true-color image of the core of A 2218 (blue: HST F450W, green: HST F814W and red: WHT/INGRID $K_{\mathrm{S}}$ ). The $850 \mu \mathrm{m}$ sub-mm image from SCUBA is overlayed as white contours. The three images of the multiply imaged sub-mm galaxy are annotated as A, B and C. The sub-mm contour at the top left corresponds to a $z=4.04$ sub-mm galaxy discussed in Knudsen et al. (2010). Two other galaxies at $z \sim 2.5$, are identified: the single-image \#273 and the fold-image \#384 and its counter image \#468. The yellow line shows the critical line at $z=2.515$. (Right) Panel of $10^{\prime \prime} \times 10^{\prime \prime}$ images showing the INGRID $K_{\mathrm{S}}$-band (left column) and HST true-color image from F450W/F606W/F814W (right column) of the four sub-mm sources in the core of A 2218. Note how each of the sub-mm sources, SMM J16359+6612.6, SMM J16359+6612.4 and SMM J16358+6612.1, comprises a NIR source $(\gamma)$ which is bracketed by two features in the F814W image ( $\alpha$ and $\beta$ ). (From Kneib et al. 2004)

forming galaxy appear to be similar to those seen in local galaxies even though the energetics are unlike anything found in the present-day Universe.

In the sub-mm domain, lensing has proven to be truly useful in revealing details about high-redshift sources that would otherwise be impossible, even with the next generation of large aperture telescopes.

\subsection{Mid-infrared lensing survey}

In the late 1990s, the ISOCAM camera on the ESA Infrared Space Observatory (ISO) targeted a number of massive cluster lenses. The motivation for these observations was to probe the faint and distant Mid-Infrared galaxy population and their contribution to the cosmic mid-infrared background radiation. In particular a few wellknown massive cluster lenses were imaged deeply by ISO at 7 and 15 micron. The deepest ISO observation of a cluster targeted Abell 2390 (Altieri et al. 1999). Crossidentification of the numerous mid-infrared sources with optical and near-infrared data showed that almost all 15 micron sources were identified as lensed distant galaxies. These observations allowed the computation of number counts in both the 7 and 15 micron bands and led to the ruling out of non-evolutionary models, and favoring 

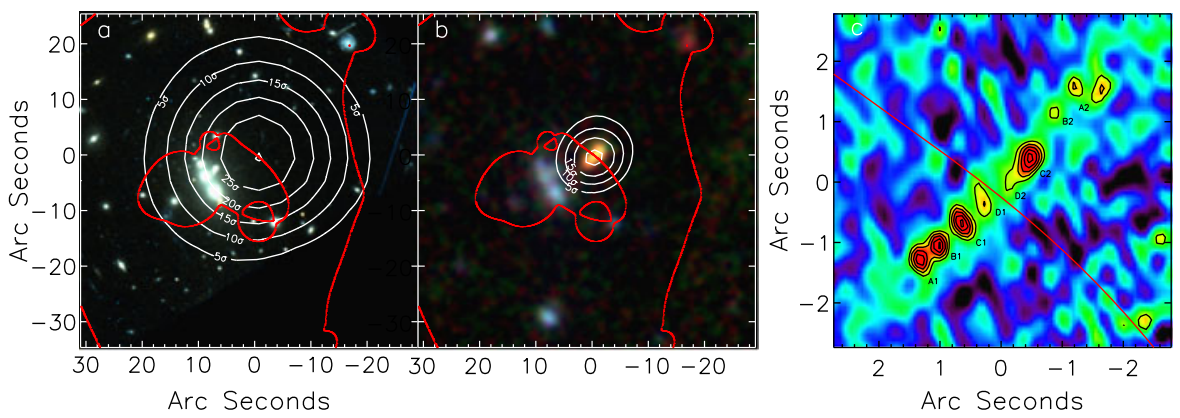

Fig. 34 (Left) Hubble Space Telescope V, I -band color image of MACSJ2135-010217 with white contours denoting the 870 micron emission of SMMJ2135-0102 with flux $106.0 \pm 7.0 \mathrm{mJy}$ as observed by APEX/LABOCA. The optical counterpart is faint with $I_{\mathrm{AB}}=23.6 \pm 0.2$. The solid red lines denote the $z=2.326$ radial and tangential critical curves from the best-fit lens model. (Center) True color IRAC 3.6, 4.5, 8.0 micron image of the cluster core with contours denoting the 350 micron emission from APEX/LABOCA. The mid-infrared counterpart is clearly visible as an extended red galaxy centered at the sub-mm position. (Right) SMA 870 micron image of the galaxy. The map shows eight individual components, separated by up to $4^{\prime \prime}$ in projection. The red line is the same $z=2.326$ radial critical curve. Components (A, B, C, D) represent two mirror images of the galaxy, each comprising four separate emission regions reflected about the lensing critical curve. Figure from Swinbank et al. (2010)

very strong number count evolution. By combining the data on three massive clusters (Abell 370, Abell 2218 and Abell 2390), Metcalfe et al. (2003) detected a total of 145 mid-infrared sources, and after a very careful lensing correction derived the intrinsic counts of the background source population. It was found that roughly $70 \%$ of the 15 micron sources are lensed background galaxies. Of sources detected only at 7 micron, $95 \%$ are cluster galaxies in this sample. Of the 15 sub-mm sources already identified within the mapped regions of the three clusters, 7 were detected at 15 micron. Flux selected subsets of the field sources above the $80 \%$ and $50 \%$ completeness limits were used to derive source counts to a lensing corrected sensitivity level of 30 micro-Jy at 15 micron, and 14 micro-Jy at 7 micron. The source counts, corrected for the effects of completeness, contamination by cluster sources and lensing, confirmed and extended earlier findings of an excess by a factor of ten in the 15 micron population with respect to source models with no evolution, with a redshift distribution that spans between $z=0.4$ and $z=1.5$.

\subsection{Lensed extremely red objects}

The benefits of lensing have also been used to search for Extremely Red Objects (EROs) behind a sample of 10 X-ray luminous galaxy clusters (Smith et al. 2002a, $2002 b$ ) imaged by both the WFPC2 camera (using F702W filter) and UKIRT in the K-band. EROs are galaxies with $R-K>5.3$ as defined by Daddi et al. (2000) as the criterion to select distant elliptical galaxies. The other more stringent definition with $R-K>6$ was adopted by Thompson et al. (1999). In these clusters a total of about 60 EROs have been identified so far allowing the number counts of these rare objects to be computed down to about 2 magnitudes fainter than previous work published at that time. The exploitation of the lensing effect has also permitted a more accurate 
Fig. 35 True color $R, K$ view $\left(50^{\prime \prime} \times 50^{\prime \prime}\right)$ of the Abell 68 cluster core (combining HST and near-infrared UKIRT data). The bright elliptical galaxy in the center of the frame is the central galaxy of the cluster. Three images of ERO J003707 are clearly visible and are marked as A, B and C. Each image comprises a central red "bulge", surrounded by numerous fainter blue knots of current or recent star formation (from Smith et al. 2002a)

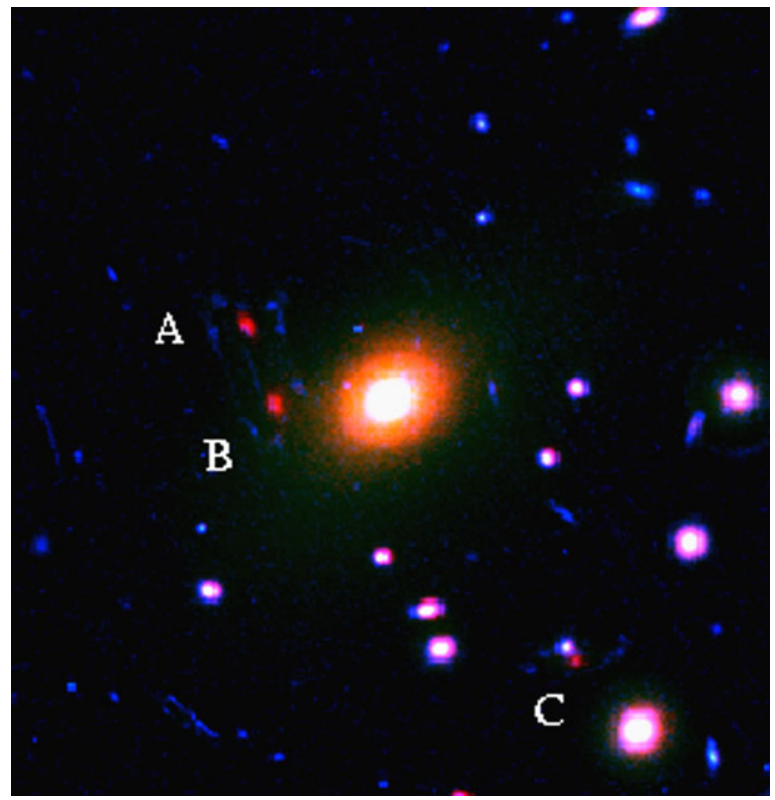

study of the morphology of these peculiar galaxies, revealing in some cases spectacular disky components already in place at fairly early times (Fig. 35). In particular, for the case of the multiply imaged ERO J003707, morphological and photometric analyses reveal an $L^{*}$ early-type disk-galaxy. It has been estimated that $\sim 10 \%$ of EROs with $R-K>5.3$ and $K<21$ have similar properties. The unique association of passive EROs with elliptical galaxies therefore appears to be too simplistic and has been reconsidered. More recent work on searching for lensed EROs was conducted in A1835 and AC114 (Schaerer et al. 2007) taking advantage of complete wavelength coverage including HST, ground based and Spitzer data. They found in these observations that most of the EROs were, in fact, young dusty starbursts at $z \sim 2-3$.

\subsection{Lensed Lyman- $\alpha$ emitters}

One of the exciting current ventures is to map the critical region of massive clusters (Fig. 36) in order to search for Lyman-alpha emitters at very high redshifts $(z>4)$, compute their number density, derive their luminosity function and therefore characterize this population. By pushing to very high redshift $z>7$ one should get closer to re-ionization, and the increase in neutral gas content of the Universe should block Lyman- $\alpha$ photons. Thus at some point one should expect a strong evolution of the Lyman- $\alpha$ emitter luminosity function. Two approaches have been pursued in the search for Lyman- $\alpha$ emitters, a direct search through intense spectroscopy, and an indirect one that relies on conducting narrow-band imaging searches in which the wavelength range is tuned to probe a specific redshift window. 


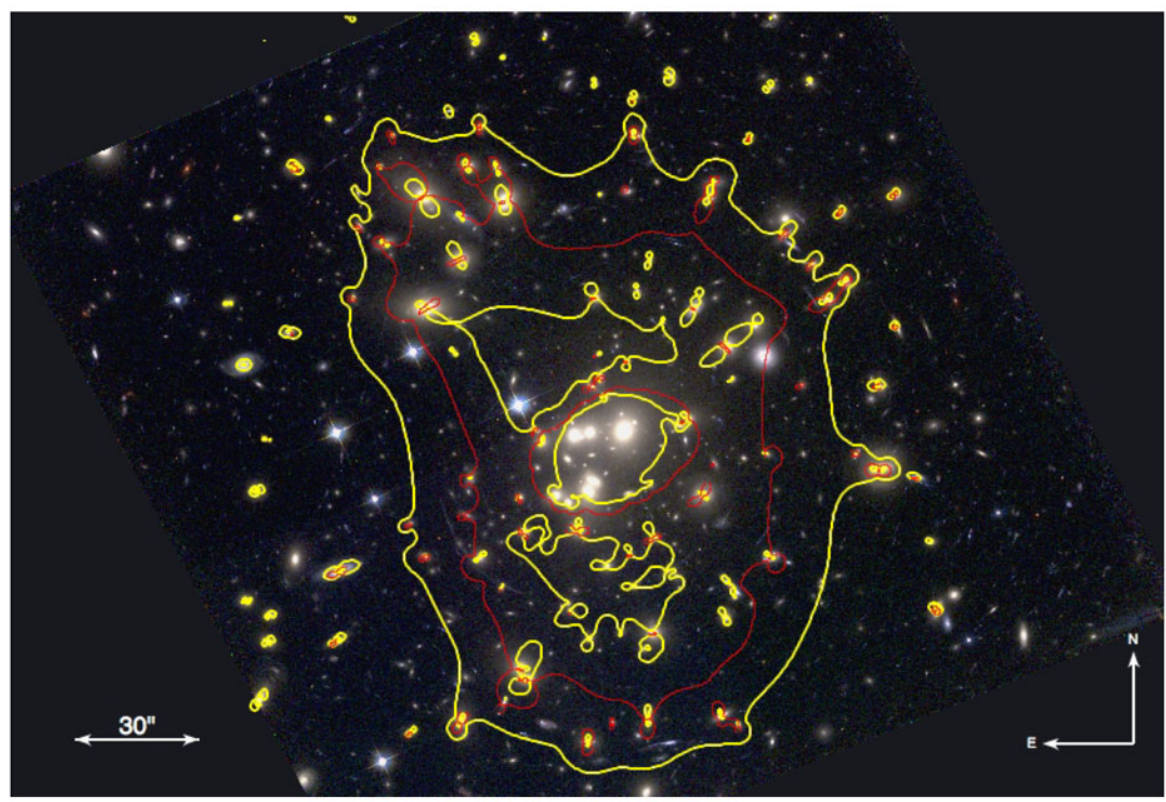

Fig. 36 The high magnification critical region in the core of the massive cluster Abell 1689. Red lines are the critical line for $z=7$ (both radial and tangential lines are shown) and the yellow contours delimit the regions of the sky having a magnification larger than a factor of ten

\subsubsection{Spectroscopic critical line mapping}

The first dedicated critical line mapping using spectroscopy was conducted at Keck, using the long-slit mode of LRIS (Sand et al. 2004). Nine intermediate redshift, massive clusters with good lensing mass models were carefully selected, and a number of long-slit observations was conducted, sliding the long slit across the critical line region thus achieving magnification factors generally greater than 10 . Eleven emission-line candidates were located in the range $2.2<z<5.6$ with Lyman- $\alpha$ as the line identification. The selection function of the survey takes into account the varying intrinsic Lyman- $\alpha$ line sensitivity as a function of wavelength and sky position. By virtue of the strong magnification factor, these measurements provide constraints on the Lyman- $\alpha$ luminosity function to unprecedented limits of $10^{40} \mathrm{erg} / \mathrm{s}$, corresponding to a star formation rate of $0.01 \mathrm{M}_{\odot} / \mathrm{yr}$. Combining these lensing results with other surveys, limited to higher luminosities, Sand et al. (2004) argue that there exists evidence for the suppression of star formation in low-mass halos, as predicted in popular models of galaxy formation. The highest redshift Lyman- $\alpha$ emitter discovered in this survey is the $z=5.576$ pair in the cluster Abell 2218 (Ellis et al. 2001). High-resolution spectroscopic follow-up confirmed the lensing hypothesis of the LRIS discovery by identifying the second image. The unlensed source appears to be a very faint source with $(I \sim 30)$ and is compact in nature $\left(<150 h_{65}^{-1} \mathrm{pc}\right)$. This source is a promising candidate for an isolated $\sim 10^{6} \mathrm{M}_{\odot}$ system seen likely producing its first generation of stars close to the epoch of re-ionization. 
Pushing to higher redshifts than $z>7$ requires an infrared spectrograph. In a challenging experiment, Stark et al. (2007) blindly surveyed the critical line region of nine massive clusters using the Keck/NIRSPEC long slit. The magnification boost ranges from 10 to $50 \times$ for a background galaxy between $\sim 8<z<\sim 10$, thus pushing the sensitivity limits to unprecedented low fluxes $\left(10^{41}-10^{42} \mathrm{erg} / \mathrm{s}\right)$ for this redshift range. This survey identified six promising $(>5 \sigma)$ candidate Lyman- $\alpha$ emitters that lie between $z=8.7$ and $z=10.2$. Lower redshift line interpretations were mostly excluded through the non-detection of secondary emission in further spectroscopy undertaken with LRIS and NIRSPEC. Nonetheless, it is considered plausible that at least two of the candidates are likely at $z \sim 9$. If true, then given the small volume surveyed, this suggests there is an abundance of low-luminosity star-forming sources at $z \sim 8-10$, which could provide a significant proportion of the UV photons necessary for cosmic re-ionization. A parallel study was conducted using the SINFONI 3D spectrograph at VLT, and three of the Keck/NIRSPEC candidates were re-observed-as part of the SINFONI critical line mapping program. However, no confirmation of the Keck/NIRSPEC detected lines was found, casting some doubt on the real redshifts of these particular sources. The results of this survey are presented in Clement et al. (2011). Future 8-10 m class instruments such as MUSE in the visible and MOSFIRE, KMOS and EMIR in the near infrared should, thanks to their higher multiplexing, provide new opportunities to further conduct critical line surveys and find more robustly numerous high-redshift Lyman- $\alpha$ emitters.

\subsubsection{Narrow-band searches}

An alternative to direct spectroscopy of Lyman- $\alpha$ emitters, is to conduct narrowband imaging. Although this technique has been very popular in blank fields, only a few such observations have been conducted in the direction of cluster cores. $\mathrm{Hu}$ et al. (2002) have discovered a redshift $z=6.56$ galaxy lying behind the cluster Abell 370 (Fig. 37). The object nicknamed HCM-6A was found in a narrow-band imaging survey using a $118 \AA$ bandpass filter centered at $9152 \AA$ using LRIS on the Keck telescope. At the time of discovery, HCM-6A was the first galaxy to be confirmed at redshift $z>6$ (its observed equivalent width is $190 \AA$, with a flux of $2.7 \times 10^{-17} \mathrm{erg} / \mathrm{cm}^{2} / \mathrm{s}$ ). Using the detailed lensing model of this cluster, a lensing amplification factor of 4.5 was estimated as the source is situated about 1 arcminute away from the cluster center. This discovery suggested that the re-ionizing epoch of the Universe lies beyond $z \sim 6$.6. Follow-up of this source with Spitzer (Chary et al. 2005) and in millimeter with MAMBO-2 (Boone et al. 2007, which provided an upper limit at $1.2 \mathrm{~mm}$ ) have helped derive some physical parameters with relatively high accuracy considering the distance of this source. Even more ambitious was the narrow J-band filter NB119 survey (corresponding to Lyman- $\alpha$ at $z \sim 9$ ) nicknamed the ' $z$ equals nine' (ZEN) survey conducted toward three massive lensing clusters: Abell clusters A1689, A1835 and AC114 (Willis et al. 2008). However, no sources consistent with a narrow-band excess were found and no detection in bluer deep optical was reported. The total coverage of the ZEN survey sampled a volume at $z \sim 9$ of approximately 1700 co-moving $\mathrm{Mpc}^{3}$ to a Ly- $\alpha$ emission luminosity of $10^{43} \mathrm{erg} / \mathrm{s}$. The limits from this survey still offer the best constraints at this redshift. 


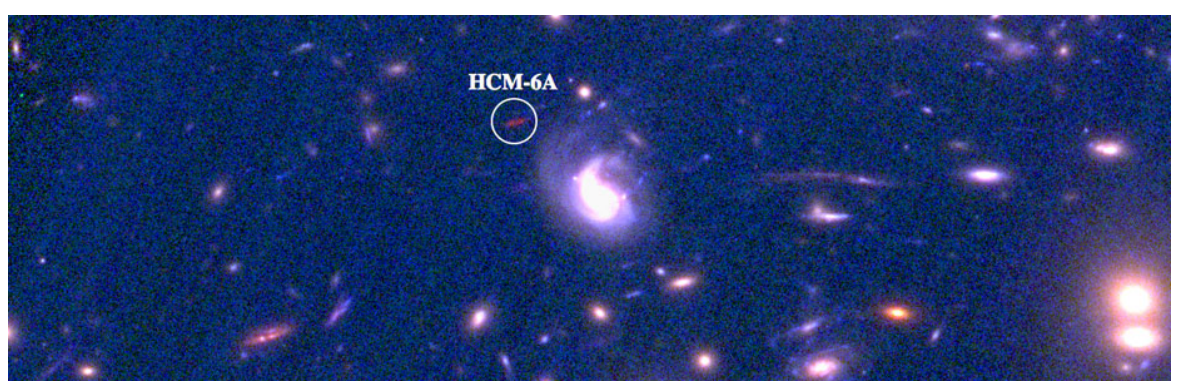

Fig. $37 H S T$ (ACS+WFC3) color $V, I, J$ view of the HCM-6A $z=6.56$ Lyman- $\alpha$ emitter located near the core of the Abell 370 cluster. Note the strong detection in the F110W (J-band) filter and its bimodal light distribution. The region shown covers $60^{\prime \prime} \times 20^{\prime \prime}$

\subsection{Lyman-break galaxies}

As the number density of Lyman-break galaxies (at $3<z<3.5$ ) is typically half a galaxy per square arcminute down to $R=25$ (Steidel et al. 1996), a massive cluster will likely magnify one of them, and if we consider a wider range in redshift, the probability that one of them is multiply imaged is almost guaranteed. The first giant arc in $\mathrm{Cl} 2244-04$ at $z=2.24$ is considered to be the first Lyman-break galaxy detected in a cluster lens, although at the time of its discovery in the late 1980s, this galaxy was not classified as such. As part of the CNOC survey, Yee et al. (1996) identified a "proto-galaxy" at $z=2.72$, the galaxy cB58 in the cluster MS1512+36. Interestingly, they assumed that this object although being very close to the cluster center was unlikely to be lensed. But soon after, Seitz et al. (1998) demonstrated that cB58 was highly lensed, magnified by more than a factor of 50 , thanks to the identification of its counter image. The estimate of such a high magnification led to a number of followup studies at high spectral resolution to further constrain the physical properties of this high-redshift galaxy (e.g. Pettini et al. 2002). At about the same time, the triple arc at $z=2.515$ in Abell 2218 (Ebbels et al. 1996) was the first recognized Lymanbreak galaxy lensed by a massive cluster, however, its magnification is only $\sim 15 \times$, much less than that of cB58. Shortly afterward in the course of a spectroscopic cluster galaxy survey of MS1358+62, Franx et al. (1997) discovered a Lyman-break galaxy at $z=4.92$ multiply imaged by the cluster. Further study and modeling by Swinbank et al. (2010) derived a magnification factor for the brightest image of $12.5 \pm 2$. At the time of discovery the arc in MS1358+62 was the most distant galaxy known. In the massive cluster Abell 2390, Frye and Broadhurst (1998) and Pelló et al. (1999) independently found a $z=4.04$ pair, strongly lensed by the cluster. These high-redshift discoveries have demonstrated the potential of discovering even higher redshift galaxies lensed by massive clusters. Thanks to deep F850LP/ACS data, following up the $z=5.56$ Lyman- $\alpha$ galaxy pair of Ellis et al. (2001), Kneib et al. (2004) found an $i$-band dropout detected in $z$-band (see Fig. 38). Detection with NICMOS confirmed a $z \sim 6.8$ redshift, however, a NIRSPEC/Keck spectrum failed to detect a Lyman- $\alpha$ line, but Spitzer IRAC 3.6 and 4.5 micron detections (Egami et al. 2005) provided strong constraints on the age of the underlying stellar population, making it one of the best studied objects at this redshift. 


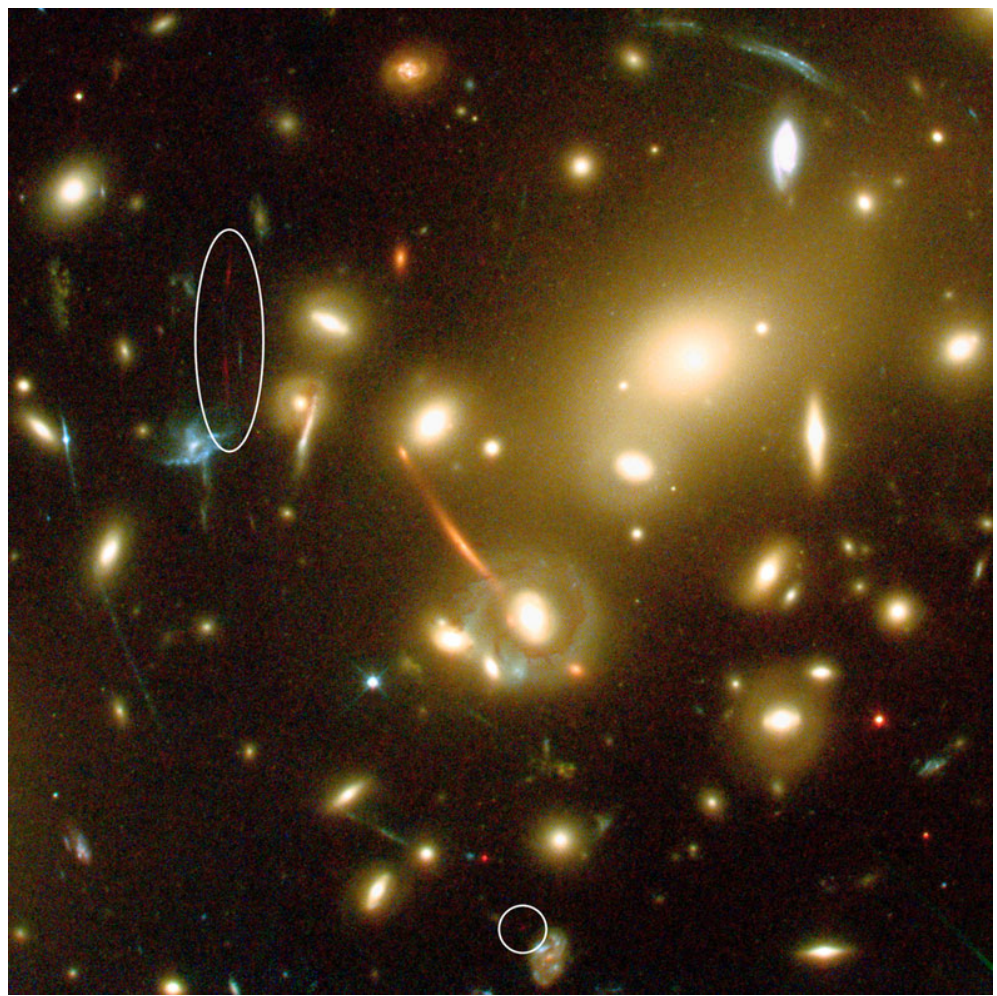

Fig. 38 HST ACS color $V, I, z$ view of Abell 2218 showing the triply imaged Lyman-break galaxy at $z \sim 6.8$ (Kneib et al. 2004)

Using the ESO/VLT instruments FORS and ISAAC, a deep imaging survey of the clusters AC114 and A1835 was conducted by Richard et al. (2006) to search for lensed optical and near-infrared dropout galaxies. In this work, they identified 26 optical dropout candidates in both A1835 and AC114 (with $H \sim 23.5-24.0$ ). Half of these candidates show an SED compatible with star-forming galaxies at $z>6$, and 6 of them are likely intermediate-redshift extremely red objects based on luminosity considerations. With this dataset a first attempt was made to characterize the luminosity function of these high-redshift galaxies, which are not well constrained by deeper HST/NICMOS observations of the HUDF (Hubble Ultra Deep Field). This work lead to the study by Richard et al. (2008) of a further six massive clusters with HST using the NICMOS camera and complemented by Spitzer observations. The survey yielded $10 \mathrm{z}$-band and $2 \mathrm{~J}$-band dropout candidates to photometric limits of $J_{110} \sim 26.2 \mathrm{AB}$ $(5 \sigma)$. By taking into account the magnifications afforded by the clusters, they probed the presence of $z>7$ sources to unlensed limits of $J_{110} \sim 30 \mathrm{AB}$, fainter than those charted in the HUDF. Taking into account the various limitations of this work, they concluded that about half of the sample of z-band dropouts are at high redshift. An ambitious infrared spectroscopic campaign undertaken with the NIRSPEC spectrograph at the Keck Observatory for seven of the most promising candidates failed to detect any Ly- $\alpha$ emission. 
Behind Abell 1689, using HST/NICMOS Bradley et al. (2008) found a bright $H=24.7 z \sim 7.6$ galaxy candidate: A1689-zD1. This source is 1.3 mag brighter than any known z850-dropout galaxy (thanks to a cluster magnification factor of $\sim 9.3 \times)$. Nevertheless, no spectroscopic observations have yet confirmed the redshift of this candidate. In the more recent years, discoveries have been reported using either the ground based ESO/Hawk-I infrared imager (Laporte et al. 2011) or the new WFC3 camera installed in May 2009 onboard HST (Bradley et al. 2011; Kneib et al. 2011; Paraficz et al. 2011). In the long term, the James Webb Space Telescope (JWST) and the Extremely Large Telescopes will uncover large numbers of these very high-redshift systems, enabling the study of their sizes, morphologies and physical parameters (e.g. Wyithe et al. 2011; Salvaterra et al. 2011).

\subsection{Far infrared lensing surveys}

With the launch of the Herschel Space Observatory in May 2009, a new window to the Universe has been opened. The Herschel Lensing Survey (HLS) conducted deep PACS and SPIRE imaging of 44 massive clusters of galaxies. These observations complement the observation of 10 massive clusters by the GTO teams. In particular, it is foreseen that the strong gravitational lensing power of these clusters will enable penetration through the confusion noise, which sets the ultimate limit on our ability to probe the Universe with Herschel. Although the analysis of this large dataset is still in progress, some early results were presented in the AandA Herschel special issue in spring 2010. In particular, Egami et al. (2010) summarized the major results from the science demonstration phase observations of the Bullet cluster $(z=0.297)$. The study of two strongly lensed and distorted galaxies at $z=2.8$ and 3.2 and the detection of the Sunyaev-Zel'dovich (SZ) effect increment of the cluster with the SPIRE data have been reported.

By looking at Abell 2218, Altieri et al. (2010) studied the population of intrinsically faint infrared galaxies that lie below the sensitivity and confusion limits using ultra-deep PACS 100 and 160 micron observations (Fig. 39). They derived (unlensed) source counts down to a flux density of $1 \mathrm{mJy}$ at 100 micron and $2 \mathrm{mJy}$ at 160 micron. In particular, the slope of the counts below the turnover of the Euclidean-normalized differential curve could be constrained in both bands and was found to be consistent with most of the recent evolutionary models. By integrating the number counts over the flux range accessed by exploiting lensing by Abell 2218 they retrieved a cosmic infrared background surface brightness of $\sim 8.0$ and $\sim 9.9 \mathrm{nW} / \mathrm{m}^{2} / \mathrm{sr}$, in the 110 and 160 micron bands, respectively. By combining the Abell 2218 results with wider/shallower fields, the source fluxes correspond to $\sim 60 \%$ and $\sim 90 \%$ of the DIRBE cosmic infrared background at 100 and 160 micron. These first Herschel results from HLS and the GTO sample will certainly expand as the data are getting analyzed and we can envision numerous follow-up observations at optical and near-infrared wavelengths as well as with ALMA.

As part as the second call for observations another interesting lensing search has been implemented in the Herschel observing program to find a larger number of exceptionally bright lensed galaxies such as the one found by Swinbank et al. (2010). 

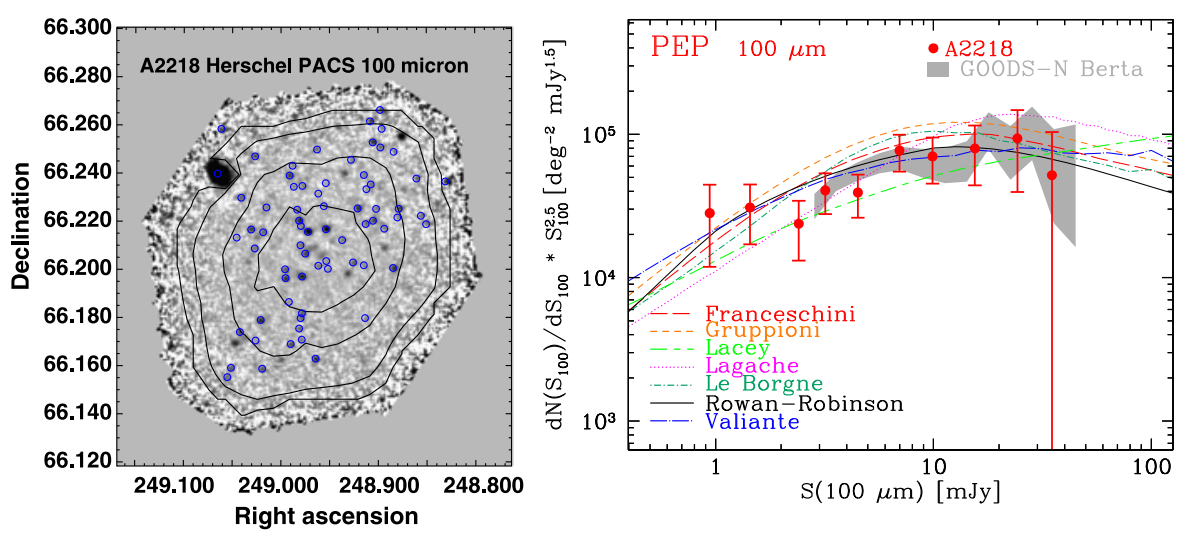

Fig. 39 (Left) PACS 100 ?m map toward Abell 2218 with lensed and foreground sources marked with blue circles, other sources are identified cluster members. Overlaid contours in black show the rms contours at 0.7, 1.0, 2.0 and $4.0 \mathrm{mJy}$. (Right) Number counts at 100 micron with lensing correction (red filled circles), normalized to the Euclidean slope, against the prediction of various synthetic counts models. Errors refer to pure Poisson statistics at $68 \%$ C.L. GOODS-N counts are contrasted in the shaded area (from Berta et al. 2010). (Figures from Altieri et al. 2010)

This project is aiming to conduct a SPIRE snapshot survey of 300 X-ray-selected massive galaxy clusters to discover the few extreme magnified objects that will then be very easy to follow-up at various wavelengths for an in-depth study. Although this survey is not yet finished, a number of highly amplified SPIRE sources have been identified, and intensive multi-wavelength follow-up are in progress.

\subsection{Cluster lensed supernovae}

In the last two decades, Supernovae ( $\mathrm{SNe}$ ) have been used for several astrophysical and cosmological applications. In particular, core collapse SNe trace the star formation history while the standard candle property of Type Ia SNe can be used for probing the expansion history of the Universe (e.g. Riess et al. 1998; Perlmutter et al. 1999; Amanullah et al. 2010). One of the focus on current SNe research is to probe the distant Universe. However, one strong limitation is the light collecting power of existing telescopes.

A possible alternative to current investigation is to target these $\mathrm{SNe}$ in the field of view of massive clusters. Although the idea is not new and was first discussed by Narasimha and Chitre (1988) and then by Kovner and Paczynski (1988), it is only recently that $\mathrm{SNe}$ observations in cluster fields has become more popular (Kolatt and Bartelmann 1998; Sullivan et al. 2000; Gal-Yam et al. 2002). The most interesting locations are of course the strong lensing regions of clusters where the amplification is the largest, and were $\mathrm{SNe}$ could be multiply imaged offering the possibility to measure the time delay between the different images.

At first SN searches were done at optical wavelengths where SNe typically emit most of their light. For example, Gal-Yam et al. (2002) using archival HST imaging of 9 clusters, in which they discovered two or three likely cluster SNe and three other $\mathrm{SNe}$, with one background to a cluster at redshift $z=0.985$. More recently, Sharon 
et al. (2010) in a dedicated SNe HST multi-epoch ACS I-band survey of 16 massive clusters (ranging from $z=0.5$ to $z=0.9$ ) have discovered $24 \mathrm{SNe}$, with eight of them being background to these clusters (the highest $\mathrm{SN}$ redshift found is at $z=1.12$ ). However, none of those lensed $\mathrm{SNe}$ are in the regions of multiple images. At even higher cluster redshift $z \sim 1$, the Supernova Cosmology Project (PI: Perlmutter) has targeted 25 clusters through an HST multi-epoch program in which nine clusters and twenty other (foreground or background) SNe have been discovered (Dawson et al. 2009). However, the main focus of these cluster multi-epoch surveys was essentially geared toward the discovery and study of cluster type Ia SNe, and thus were not optimized to benefit from the cluster lens magnification.

On the contrary, Gunnarsson and Goobar (2003) presented the feasibility of detecting high-z SNe along the line of sight of massive clusters, in particular focusing on the SNe detection in the near infrared. Using a dedicated VLT/ISAAC multi-epoch SN survey, Stanishev et al. (2009) and Goobar et al. (2009) reported the discovery of a highly amplified SN at $z \sim 0.6$ behind the well-studied Abell 1689 cluster. More recently, using the new VLT/Hawk-I infrared camera, Amanullah et al. (2011) found one of the most distant SNe ever found at $z=1.703$ (measured through X-Shooter spectroscopy of the galaxy host) thanks to the large magnification $(\sim 4.3 \pm 0.3)$ of the massive cluster Abell 1689. This study demonstrated that further SNe follow-up may lead to important new discoveries.

\section{Cosmological constraints from cluster lensing}

In this section, we discuss three powerful cluster lensing based methods at various stages of development and application that may provide competitive and important constraints on cosmological parameters. These are: cosmography using several sets of multiple images lensed by the same cluster; the abundance of arcs and the statistics of lensed image triplets. For cosmography and triplet statistics purely geometric constraints are obtained via the ratio of angular diameter distances, whereas the abundance of arcs provides potentially strong constraints on the growth of structures and primordial non-Gaussianity.

\subsection{Cosmography with multiple images}

Measurements of the Hubble diagram for Type Ia Supernovae (SNIa) (Riess et al. 1998; Perlmutter et al. 1999) combined with constraints from the Wilkinson Microwave Anisotropy Probe (WMAP5) (Spergel et al. 2003), cosmic shear observations (Bacon et al. 2000; Kaiser et al. 2000; van Waerbeke et al. 2000; Wittman et al. 2000; Semboloni et al. 2006), cluster baryon fraction (Allen et al. 2004), cluster abundances (Vikhlinin et al. 2009) and baryon acoustic oscillations (BAO) from galaxy surveys (Efstathiou et al. 2002; Seljak et al. 2005; Eisenstein et al. 2005) suggest that 72\% of the total energy density of the Universe is in the form of an unknown constituent with negative pressure - the so-called dark energy, which powers the measured accelerating expansion. These observations probe the equation-of-state parameter $w_{\mathrm{X}}$, defined as the ratio of pressure to energy density, through its effect on the expansion history of the Universe and the growth of structures. 
Constraining the geometry and matter content of the Universe using multiple sets of arcs has been explored in cluster lenses using different techniques (Paczynśki and Gorski 1981; Link and Pierce 1998; Cooray 1999a, 1999b; Golse et al. 2002; Sereno 2002; Sereno and Longo 2004; Soucail et al. 2004; Dalal et al. 2005; Meneghetti et al. 2005a, 2005b; Maccio 2005; Gilmore and Natarajan 2009; Jullo et al. 2010).

As shown in Sect. 1, the lensing deflection produced in the image of a background source depends on the detailed mass distribution of the cluster as well as on the ratio of angular diameter distances. The cosmological dependence arises from the angular diameter distance ratios that encapsulate the geometry of the Universe and are a function of both $\Omega_{\mathrm{m}}$ and $\Omega_{X}$.

The most promising technique, is using multiple sets of arcs with measured redshifts. By taking the ratio of their respective Einstein radii and marginalizing over parameters of the mass distribution, one can in principle constrain the cosmological parameters $\Omega_{\mathrm{m}}$ and $\Omega_{X}$. In this method, the angular diameter distance ratios for two images from different sources defines the 'family ratio' $\Xi$, from the cosmological dependence of which constraints on $\Omega_{\mathrm{m}}$ and $w_{X}$ are extracted:

$$
\Xi\left(z_{\mathrm{L}}, z_{\mathrm{s} 1}, z_{\mathrm{s} 2} ; \Omega_{\mathrm{m}}, \Omega_{\mathrm{X}}, w_{X}\right)=\frac{D\left(z_{\mathrm{L}}, z_{\mathrm{s} 1}\right)}{D\left(0, z_{\mathrm{s} 1}\right)} \frac{D\left(0, z_{\mathrm{s} 2}\right)}{D\left(z_{\mathrm{L}}, z_{\mathrm{s} 2}\right)},
$$

where $z_{\mathrm{L}}$ is the lens redshift, $z_{\mathrm{s} 1}$ and $z_{\mathrm{s} 2}$ are the two source redshifts, and $D\left(z_{1}, z_{2}\right)$ is the angular diameter distance.

Link and Pierce (1998) showed that the cosmological sensitivity of the angular size-redshift relation could be exploited using sources at distinct redshifts and developed a methodology to simultaneously invert the lens and derive cosmological constraints. Golse et al. (2002) using simulated cluster data, showed that the recovery of cosmological parameters was feasible with at least three sets of multiple images for a single cluster. Soucail et al. (2004) then applied the technique to the lensing cluster Abell 2218 using 4 systems of multiple images at distinct redshifts, and found $\left(\Omega_{\mathrm{m}}<0.37, w_{\mathrm{X}}<-0.80\right)$ assuming a flat Universe.

Jullo et al. (2010) have presented the results of the first application of this method to the massive lensing cluster Abell 1689 at $z=0.184$ (see Fig. 40). Based on images from the Advanced Camera for Surveys $(A C S)$ aboard the Hubble Space Telescope $(H S T)$ this cluster has 114 multiple images from 34 unique background galaxies, 24 of which have secure spectroscopic redshifts (ranging from $z \sim 1$ to $z \sim 5$ ) obtained with the Very Large Telescope (VLT) and Keck Telescope spectrographs (Broadhurst et al. 2005; Limousin et al. 2007). Their parametric model has a total of 21 free parameters consisting of two large-scale potentials, a galaxy-scale potential for the central brightest cluster galaxy (BCG), and includes the modeling of 58 of the brightest cluster galaxies. The contribution of substructure in the lens plane and along the line of sight is explicitly included (see D'Aloisio and Natarajan 2011a, 2011b for a detailed discussion of the systematics). Combining the lensing derived cosmological constraints with those from X-ray clusters and the Wilkinson Microwave Anisotropy Probe 5-year data gives $\Omega_{\mathrm{m}}=0.25 \pm 0.05$ and $w_{\mathrm{X}}=-0.97 \pm 0.07$ which are consistent with results from other methods (see Fig. 41). Inclusion of this work with all other techniques available brings down the current $2 \sigma$ contours on the dark energy equation of state parameter $w_{X}$ by about $30 \%$. 


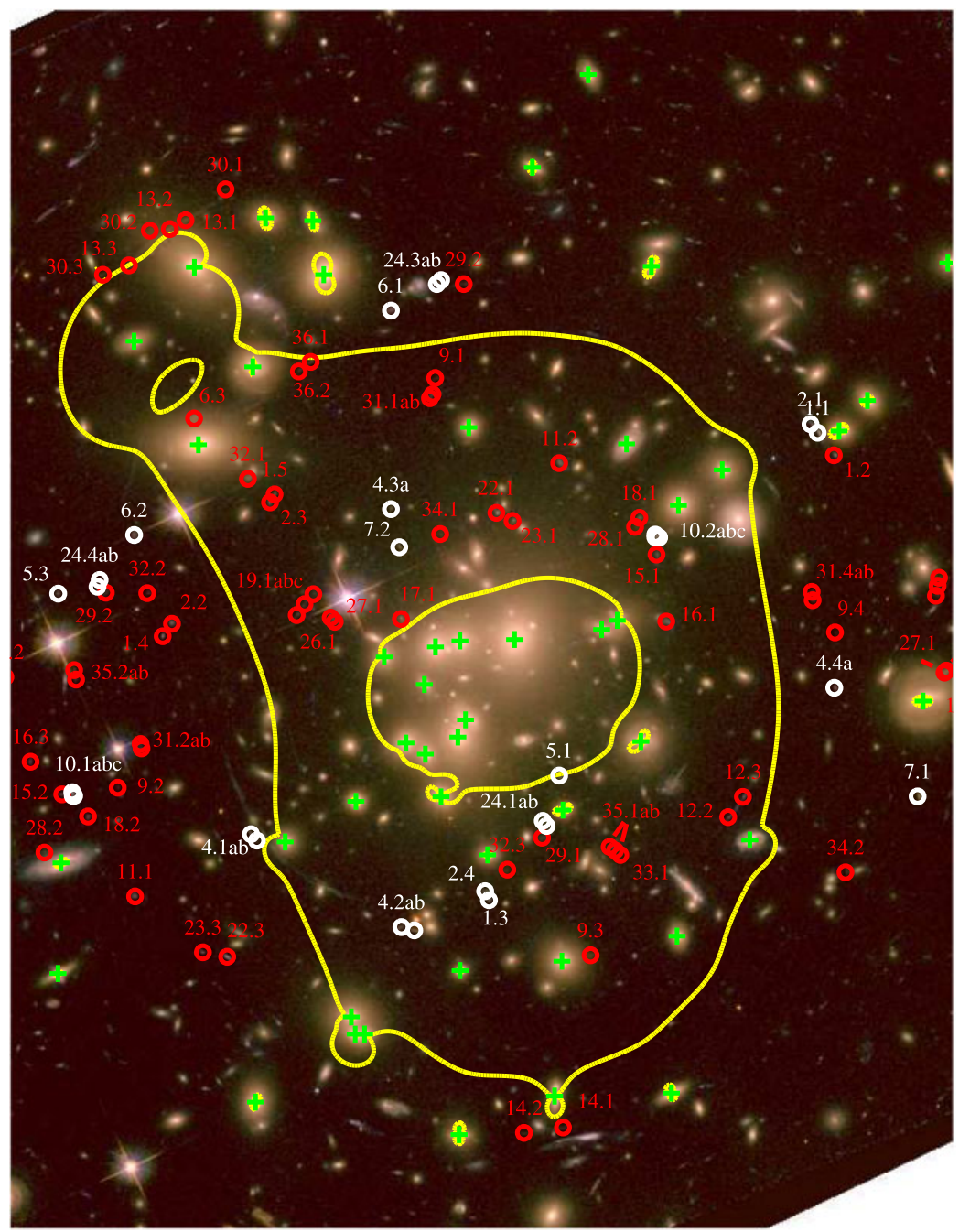

Fig. 40 The critical lines for a source at $z=3$ are over-plotted in yellow on the HST ACS image of Abell 1689. The lensing mass model used is the one from which we derived cosmological constraints. In addition to two large-scale clumps and the BCG, this model includes the contribution of 58 cluster galaxies. The positions of cluster galaxies are marked with green crosses. Over-plotted in white are the 28 multiple images arising from 12 families used in their work; the red circles mark the positions of the rejected images (figure from Jullo et al. 2010)

As with all techniques, an accurate inventory of the key systematics and their contribution to the error budget is also the challenge for this technique. The two significant current limitations arise from: (i) accounting appropriately for the lensing effect of the uncorrelated line of sight substructure (see schematic in Fig. 42) and (ii) the simplifying scaling relations assumed to relate galaxy total mass to galaxy light. However, the results from Abell 1689 are extremely encouraging and the future 
Fig. 41 The current cosmological constraints in the $\left(\Omega_{\mathrm{m}}, \Omega_{\lambda}\right.$ ) based on the best-fit model of Abell 1689 from Jullo et al. (2010): the results from combining cosmological constraints from WMAP5 + evolution of X-ray clusters + cluster strong lensing (cluster only methods); the 1 and $2 \sigma$ contours are plotted, blue contours constraints from WMAP5, pink contours X-ray clusters, orange contours cluster strong lensing

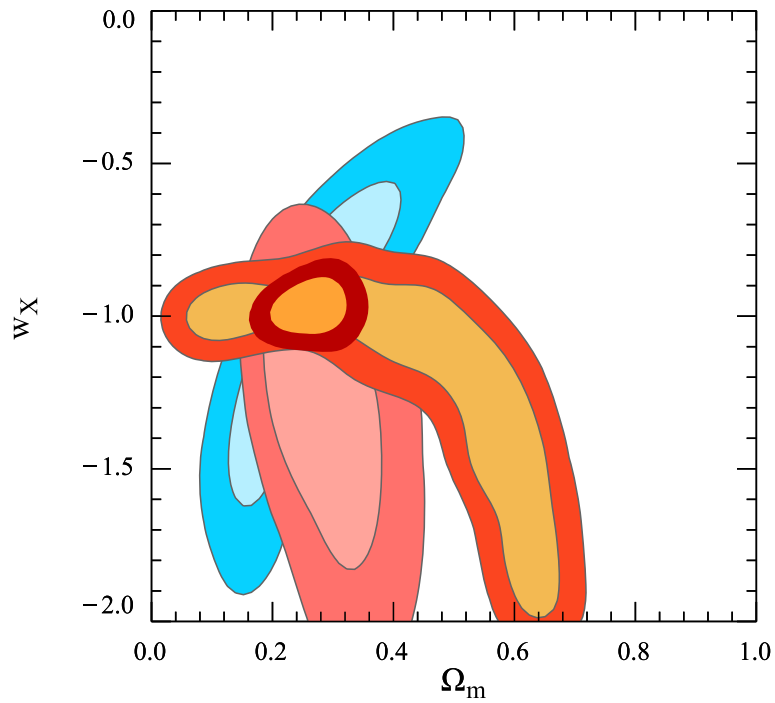

prospects for this method look promising due to the power from combining several clusters at various redshifts.

\subsection{Arc statistics and primordial non-Gaussianity}

The production of giant arcs by lensing clusters is ubiquitously observed. The abundance of massive clusters available to do so is sensitive both to the expansion history and initial conditions of the Universe. Given the scaling of the lensing efficiency with redshift, it is known that the frequency of giant-arc formation depends on the abundance and characteristics of galaxy-clusters roughly half-way to the sources. Cluster physics, cosmological effects and the properties of the high-redshift source population all play a role in determining the abundance of giant arcs, however, isolating these effects is difficult. It was originally claimed by Bartelmann et al. (1998) that the $\Lambda$ CDM model predicted approximately an order of magnitude fewer arcs than seen in observations. Subsequent studies (e.g. Zaritsky and Gonzalez 2003; Gladders et al. 2003) substantiated this claim of a 'giant-arc problem'. This mis-match between observations and the concordance cosmological model predictions suggest that either the Bartelmann et al. (1998) analysis was lacking a crucial component of properties exhibited by real cluster lenses and the source population (Williams et al. 1999) or that the concordance cosmology is in fact inconsistent with the observed abundance of giant arcs. A significant amount of effort has been expended toward understanding the most important characteristics of arc-producing clusters, and how they may not be typical of the general cluster population (e.g. Hennawi et al. 2007; Meneghetti et al. 2010; Fedeli et al. 2010). Other studies focused on effects that were not captured in early simulations. The mass contribution of central galaxies appears to have a significant effect, though not enough to entirely resolve the Bartelmann et al. (1998) disagreement alone (Meneghetti et al. 2003a, 2003b; Dalal et al. 2004). The probability of giant-arc formation increases with source redshift, 

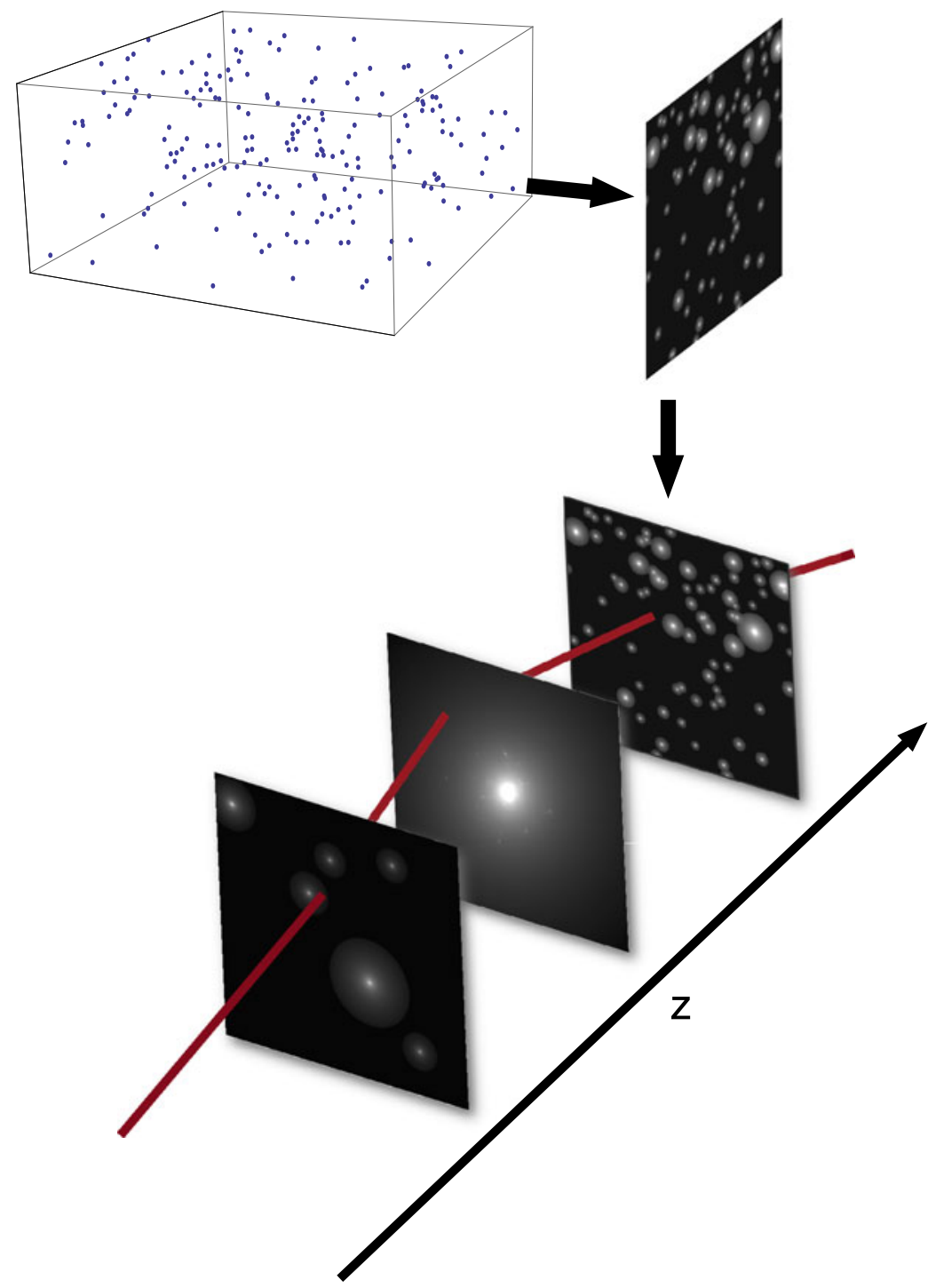

Fig. 42 Schematic diagram illustrating the creation of lens planes to quantify the lensing effects of halos along the line of sight. A rectangular slice of the Millennium Simulation box is taken and the locations of halos are projected along the long axis and analytic NFW potentials are placed on those positions. The NFW parameters are obtained through scaling relations with mass and redshift. The lens plane is inserted at the appropriate redshift and a multi-plane lensing algorithm is used to trace rays. Many lens planes between $z=0$ and $z=5$ are used to estimate the systematic errors at the positions of multiple images from the structure along the line of sight

therefore the overall abundance is sensitive to uncertainties in the high-redshift tail of the source-redshift distribution (Wambsganss et al. 2004; Dalal et al. 2004; $\mathrm{Li}$ et al. 2005). However, none of these effects can account for the observed discrepancy. On the other hand, taking into account a realistic source population and obser- 
vational effects, Horesh et al. (2005) claimed that the clusters predicted by $\Lambda \mathrm{CDM}$ have the same arc production efficiency as the observed clusters. The effects of baryonic physics, such as cooling and star formation, on central mass distributions have also been investigated (Meneghetti et al. 2010).

The amplitude of the linear matter power spectrum plays a critical role in determining how severe and if there is a giant-arc problem or not. Observations seem to be converging on $\sigma_{8} \approx 0.8$ (Fu and Stockton 2008; Vikhlinin et al. 2009; Komatsu et al. 2011), while most numerical studies on the giant-arc abundance to date have assumed $\sigma_{8}=0.9$. It is likely that adjusting $\sigma_{8}$ from 0.9 to 0.8 will lower the predicted giant-arc abundance significantly, increasing tension with observations (Li et al. 2006; Fedeli et al. 2008). The cosmological model may play a role here. In arguing that the giant-arc problem may be unavoidable if $\sigma_{8} \approx 0.8$, Fedeli et al. (2008) mention in passing that early dark energy or non-Gaussian initial conditions may provide "a way out." The effects of dark energy on giant-arc statistics have been investigated in Bartelmann et al. (2003), Maccio (2005), Meneghetti et al. (2005a, 2005b), and Fedeli and Bartelmann (2007). On the other hand, the possible effects of non-Gaussian initial conditions have only been explored recently by D'Aloisio and Natarajan (2011a, 2011b). They argue that primordial non-Gaussianity (PNG) can affect the probability of giant-arc formation in at least two ways. ${ }^{7}$ First, PNG can lead to an enhanced or diminished abundance of galaxy clusters, depending on the particular model (e.g. Matarrese et al. 2000; Lo Verde et al. 2008; Dalal et al. 2008), which would lead to a change in the number of supercritical lenses that are available in the appropriate redshift range. Secondly, PNG is expected to influence the central densities of halos (Avila-Reese et al. 2003; Oguri and Blandford 2009; Smith et al. 2010). Since lensing cross sections are sensitive to central densities, we expect corresponding changes in them as well. If a cluster lens cannot produce arcs with length-to-width ratios above some threshold, then its cross section for giant-arc production is zero. Roughly speaking, this corresponds to a minimum mass required to produce giant arcs. Owing to the effects on central densities, we expect PNG to alter this minimum mass threshold as well.

D'Aloisio and Natarajan (2011a, 2011b) quantify the impact of non-Gaussian initial conditions with the local bispectrum shape on the predicted frequency of giant arcs. Non-Gaussianity is generally expressed in terms of $f_{\mathrm{NL}}$ that characterizes the amplitude of non-Gaussianity in the primordial curvature perturbation (Komatsu and Spergel 2001). Using a path-integral formulation of the excursion set formalism, extending a semi-analytic model for calculating halo concentrations to the case of PNG, they show that massive halos tend to collapse earlier in models with positive $f_{\mathrm{NL}}$, relative to the Gaussian case, leading to enhanced concentration parameters. The converse is true for $f_{\mathrm{NL}}<0$. In addition to these effects, which change the lensing cross sections, non-Gaussianity also modifies the abundance of supercritical clusters available for lensing. These combined effects work together to either enhance $\left(f_{\mathrm{NL}}>0\right)$ or suppress $\left(f_{\mathrm{NL}}<0\right)$ the probability of giant-arc formation (see Fig. 43). Using the best value and $95 \%$ confidence levels currently available from the Wilkinson Microwave

\footnotetext{
${ }^{7}$ The PNG model considered here is the simplest one that gives rise to a non-zero 3-point correlation function.
} 
Fig. 43 The ratio of giant-arc cross sections in the case of non-Gaussian and Gaussian initial conditions. Halos have enhanced central densities in models with $f_{\mathrm{NL}}>0$. Their giant-arc cross sections are therefore increased relative to the Gaussian case and vice versa (figure from D'Aloisio and Natarajan 2011a, 2011b)

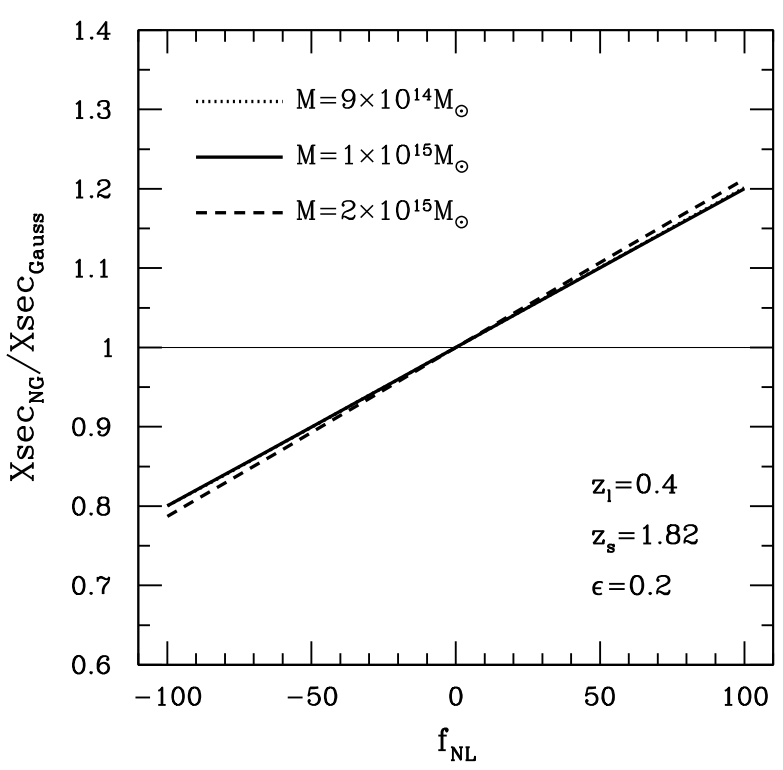

Anisotropy Probe, they report that the giant-arc optical depth for sources at $z_{\mathrm{s}} \sim 2$ is enhanced by $\sim 20 \%$ and $\sim 45 \%$ for $f_{\mathrm{NL}}=32$ and 74 , respectively. Conversely they report a suppression of $\sim 5 \%$ for $f_{\mathrm{NL}}=-10$. These differences translate to similar relative changes in the predicted all-sky number of giant arcs. Ideally the goal is to use giant-arc statistics to constrain small scale PNG. The prospects are extremely promising given upcoming all sky surveys planned by future deep wide-field imaging surveys such as foreseen by the Dark Energy Survey (DES), the Large Synoptic Survey Telescope (LSST) and future wide-field space mission (e.g. EUCLID).

\subsection{Triplet statistics}

Triplet statistics offer an interesting geometrical method that uses the weak gravitational lensing effects of clusters to constrain the cosmological parameters $\Omega_{\mathrm{m}}$ and $\Omega_{\Lambda}$ (Gautret et al. 2000). For each background galaxy, a foreground lensing cluster induces a magnification that depends on the local convergence $\kappa$ and shear terms $\gamma_{1}$ and $\gamma_{2}$ and on the cosmological parameters through the angular diameter distance ratio $D_{\mathrm{LS}} / D_{\mathrm{OS}}$. To disentangle the effects of these three quantities, the ellipticities of each triplet of galaxies located at about the same apparent position in the lens plane (although at three distinct redshifts) needs to be compared. The simultaneous knowledge of ellipticities and redshifts of each triplet enable the building of a purely geometrical estimator $G\left(\Omega_{\mathrm{m}}, \Omega_{\Lambda}\right)$ that is independent of the lens potential. This estimator $G$ has the simple form of the determinant of a $3 \times 3$ matrix built with the triplet values of $D_{\mathrm{LS}} / D_{\mathrm{OS}}$ and observed ellipticities.

When $G$ is averaged over many triplets of galaxies, it provides a global function which converges to zero for the true values of the cosmological parameters. However, in order to apply this method the various sources of statistical noise need to be quantified. The linear form of $G$ with respect to the measured ellipticity of each galaxy 
implies that the different sources of noise contributing to $G$ decrease as $1 / \sqrt{N}$, where $N$ is the total number of observed lensed galaxies. From simulations that incorporate realistic geometries and convergences for lensing clusters and a redshift distribution for galaxies, the results are promising for a sample of 100 clusters. These 100 clusters essentially need to be imaged in multiple bands to obtain accurate photometric redshifts for the triplets. With next generation cosmological surveys the observational data needed for this sample size would not be impossible to obtain.

\section{Comparison of observed lensing cluster properties with theoretical predictions}

With the growing success of gravitational lensing analysis of clusters, it has become possible to compare and test theoretical predictions against observations. With the rapid progress in high-resolution cosmological simulations of dark matter we now have a unique opportunity to directly compare properties of cluster dark matter halos derived from lensing studies. Many important physical questions with regard to the internal structures of halos, their dynamical evolution and the granularity of dark matter can now be tackled: the assembly process (role of merging sub-clusters); lensing cross sections; efficiency of lensing and super-lenses; selection effects; mass profiles; density profiles; ellipticity; alignments; abundances, and the mass concentration. We briefly outline below the results of recent studies on this topic.

\subsection{Internal structure of cluster halos}

In cosmological simulations of structure formation it is found that the density profiles of dark matter halos are well fit over many decades in mass from cluster mass scales down to dwarf galaxy scales by the Navarro-Frenk-White profile (see Appendix A.3 for details). By combining strong and weak lensing constraints, as discussed above it has become possible to probe the mass profile of the clusters on scales of $0.1-5 \mathrm{Mpc}$, thus providing a valuable test of the universal form proposed by NFW on large scales (e.g. Okabe et al. 2010; Umetsu et al. 2011). As for the inner density profile slopes there appears to be similarly a large degree of variation, some like Cl0024+1654 (Kneib et al. 2003; Tu et al. 2008; Limousin et al. 2008) adequately fit the NFW form, others like RXJ1347-11 are found to have slopes shallower than the NFW prediction (Newman et al. 2011; Umetsu et al. 2011), while others like MS2137-23 are found to have steeper slopes (Sand et al. 2004). One caveat with the NFW prediction is that the functional form is derived from dark matter only simulations, whereas in reality it is clear that baryons in the inner regions close to the $\mathrm{cD} / \mathrm{BCG}$ play a significant role both in terms of the mass budget and modifications to density profile slopes in the very center.

Lensing clusters are preferentially more significantly concentrated than all clusters (see Fig. 44 and Comerford and Natarajan 2007; but also: Broadhurst et al. 2008; Oguri et al. 2009 and Meneghetti et al. 2011) and they typically tend to be outliers on the concentration-mass relationship predicted for clusters in the $\Lambda \mathrm{CDM}$ model. The origin of this enhanced concentration parameter is likely due to: (i) high incidence of projected line of sight structures for massive lensing clusters; (ii) elongated 
Fig. 44 Observed cluster concentrations and virial masses derived from lensing (filled circles) and X-ray (open circles) measurements. For reference, the solid lines depict the best-fit power law to our complete sample and its $1-\sigma$ scatter. The lensing concentrations appear systematically higher than the $\mathrm{X}$-ray concentrations, and a Kolmogorov-Smirnov test confirms that the lensing results likely belong to a different parent distribution. This figure is from Comerford and Natarajan (2007)

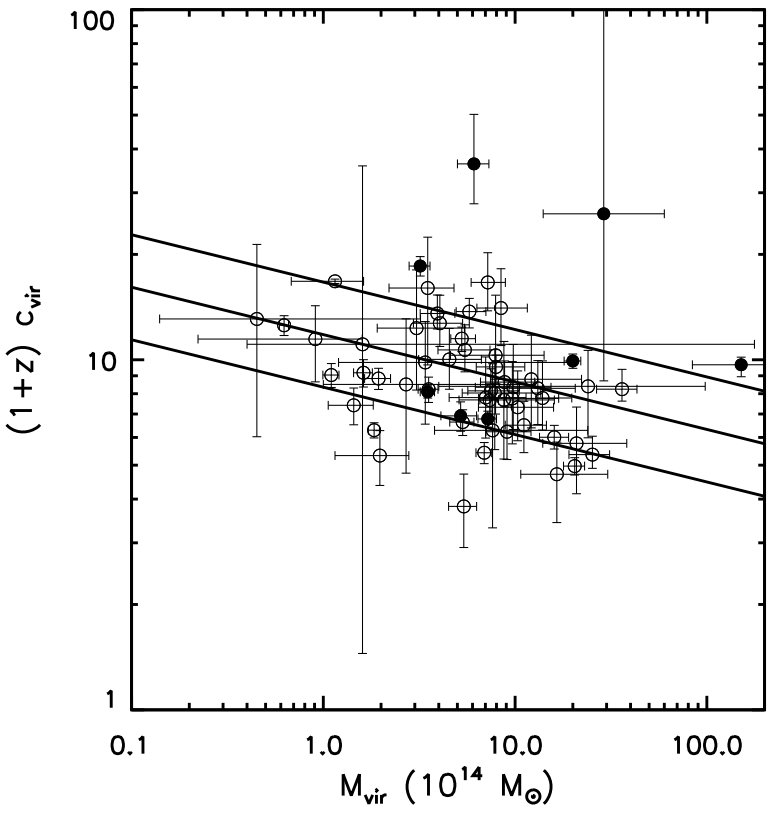

shapes that enhance lensing efficiency, factors that might observationally bias lensing selection; (iii) baryons that could play an important role in the inner regions.

\subsection{Mass function of substructure in cluster halos}

Combining observed strong and weak lensing and exploiting galaxy-galaxy lensing inside clusters, it has been possible to map the granularity of the dark matter distribution (Natarajan and Kneib 1997; Natarajan and Springel 2004; Natarajan et al. 2007) in clusters and compare them to predictions from the Millennium cosmological simulation (Springel et al. 2005). This is done by attributing local anisotropies in the observed shear field to the presence of dark matter sub-halos (Natarajan et al. 2009). The mass function thus derived for several clusters agrees well with that predicted entirely independently from high-resolution cosmological simulations of structure formation in the standard $\Lambda \mathrm{CDM}$ paradigm over the mass range $10^{11}-10^{13} \mathrm{M}_{\odot}$. The comparison was made with clusters that form in the Millennium Simulation (Springel et al. 2005). This excellent agreement of the mass function derived from these two independent methods demonstrates that there is no substructure problem (which was claimed earlier) on cluster scales in $\Lambda \mathrm{CDM}$. This is a significant result as a substructure crisis has been claimed on galaxy scales. Since $\Lambda C D M$ is a self-similar theory, if the substructure problem had been endemic to the model, it would have been replicated on cluster scales. This suggests that the substructure discrepancy on galaxy scales arises from the galaxy formation process or from some hitherto undiscovered coupling between baryons and dark matter particles. Therefore, lensing clusters have provided unanticipated insights into the dark matter model. Moving on from the Millennium Simulation, state of the art at the present time is the Mare Nostrum simulation which is promising in terms of mass resolution and larger volume probed and 
offers a new test-bed for comparison with lensing data from cluster surveys like the CLASH Hubble survey (Meneghetti et al. 2011).

\subsection{Dynamical evolution of cluster halos}

Exploiting strong and weak gravitational lensing signals inferred from panoramic Hubble Space Telescope imaging data, high-resolution reconstructions of the mass distributions are now available for clusters ranging from $z=0.2-0.5$. Applying galaxy-galaxy lensing techniques inside clusters the fate of dark matter sub-halos can now be tracked as a function of projected cluster-centric radius out to $1-5 \mathrm{Mpc}$, well beyond the virial radius in some cases. There is now clear detection of the statistical lensing signal of dark matter sub-halos associated with both early-type and late-type galaxies in clusters. In fact, it appears now that late-type galaxies in clusters (which dominate the numbers in the outskirts but are rare in the inner regions of the cluster) also possess individual dark matter halos (Treu et al. 2002; Limousin et al. 2005, 2007; Moran et al. 2006; Natarajan et al. 2009). In the case of the cluster $\mathrm{Cl} 10024+1656$ that has been studied to beyond the virial radius, the mass of a fiducial dark matter halo that hosts an early-type $\mathrm{L}^{*}$ galaxy varies from $M=6.3 \pm 2.7 \times 10^{11} \mathrm{M}_{\odot}$ within $r<0.6 \mathrm{Mpc}$, to $1.3 \pm 0.8 \times 10^{12} \mathrm{M}_{\odot}$ within $r<2.9 \mathrm{Mpc}$, and increases further to $M=3.7 \pm 1.4 \times 10^{12} \mathrm{M}_{\odot}$ in the outskirts. The mass of a typical dark matter sub-halo that hosts an $\mathrm{L}^{*}$ galaxy increases with projected cluster-centric radius in line with expectations from the tidal stripping hypothesis. Early-type galaxies appear to be hosted on average in more massive dark matter sub-halos compared to late-type galaxies. Early-type galaxies also trace the overall mass distribution of the cluster whereas late-type galaxies are biased tracers. The findings in this cluster and others are interpreted as evidence for the active redistribution of mass via tidal stripping in galaxy clusters. Upon comparison of the masses of dark matter sub-halos as a function of projected cluster-centric with the equivalent mass function derived from clusters in the Millennium Run very good agreement is found (see Fig. 45 and Natarajan et al. 2007). However, simulated subhalos appear to be more efficiently stripped than lensing observations suggest (see Fig. 46). This is likely an artefact of comparison with a dark matter only simulation. Future simulations that simultaneously follow the detailed evolution of the baryonic component during cluster assembly will be needed for a more detailed comparison. Lensing has proved to be a powerful probe of how clusters assemble and grow, and it appears that our findings ratify the $\Lambda$ CDM paradigm, hierarchical growth of structure, and the key role played by tidal stripping during cluster assembly.

\subsection{Constraints on the nature of dark matter}

While it is clear that clusters are vast repositories of dark matter, the nature of dark matter remains elusive. A plethora of astronomical observations from the early Universe to the present time are consistent with the dark matter being a cold, collisionless fluid that does not couple to baryons. However, there is potential for dark matter self-interactions and lensing observations offer a unique window to probe this further (e.g. Miralda-Escudé 2002). Limits on the dark matter interaction cross section can be 


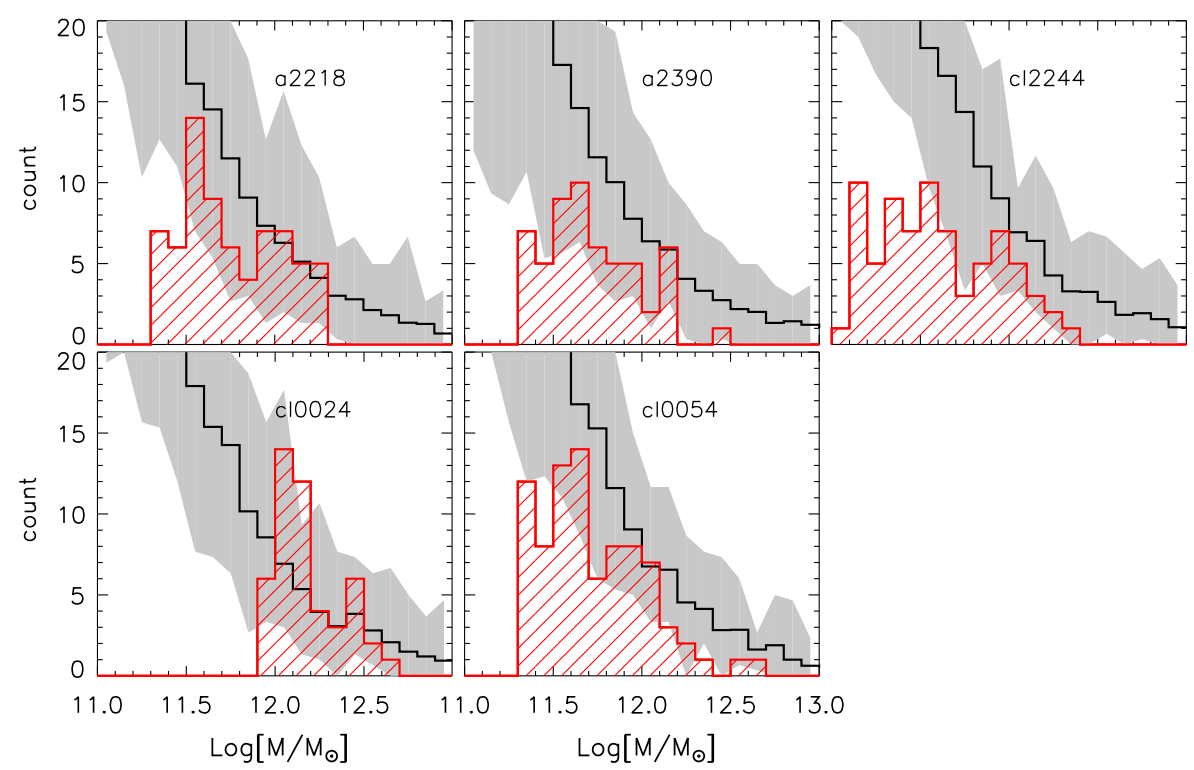

Fig. 45 Comparison between substructure mass function retrieved from the galaxy-galaxy lensing analysis (red shaded histograms) and results from haloes selected from the Millennium Simulation. The black solid line in each panel represents the average sub-halo mass function of haloes selected at the redshift of the observed lensing cluster (see text for details). The grey shaded region represents, for each value of the sub-halo mass, the min-max number of substructures found in the simulated haloes (figure from Natarajan et al. 2007)

placed from lensing observation of clusters, however, these are currently not particularly constraining or illuminating. Two distinct arguments have been used to obtain limits that strongly support the collision-less nature of dark matter. One involves the distribution of the sizes of tidally truncated sub-halos in clusters (Natarajan et al. 2002a, 2002b); and the second involves estimates from the separation between the dark matter and X-ray gas in the extreme merging system, the Bullet Cluster (Clowe et al. 2006; Bradač et al. 2006) wherein they find $\sigma / m<4 \mathrm{~g} \mathrm{~m}^{-1} \mathrm{~cm}^{2}$ assuming that the two colliding sub-clusters experienced a head-on collision in the plane of the sky. Similar results were also found from the so-called "Baby Bullet" cluster (Bradač et al. 2008). Exploring these merging clusters is certainly an avenue where lensing observations may provide constraints and insights on the nature of dark matter.

\section{Future prospects}

Since the discovery of giant arcs in the late 1980s gravitational lensing by clusters of galaxies has now become a powerful cosmological tool.

We list below some possible new avenues for the next exciting discoveries in the coming years using gravitational lensing in clusters, assuming improvements in the data quality and data volume: 


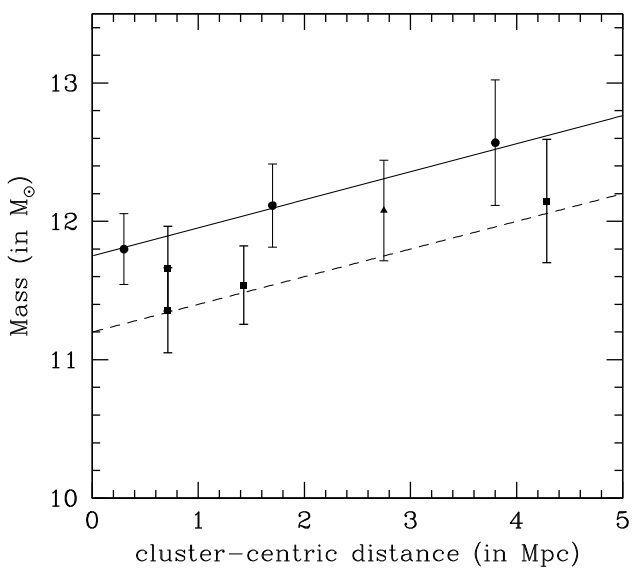

Fig. 46 Variation of the mass of a dark matter sub-halo that hosts an early-type $\mathrm{L}^{*}$ galaxy as a function of cluster-centric radius. The results from the likelihood analysis are used to derive the sub-halo mass for the galaxy-galaxy lensing results and the counterparts are derived from the Millennium Simulation with an embedded semi-analytic galaxy formation model. This enables selection of dark matter halos that host a single $\mathrm{L}^{*}$ galaxy akin to our assumption in the lensing analysis. The solid circles are the data points from the galaxy-galaxy lensing analysis and the solid squares are from the Millennium Simulation. The upper solid square in the core region marks the value of the sub-halo mass with correction by a factor of 2 as found in Natarajan et al. (2007). The solid triangle is the galaxy-galaxy lensing data point for the sub-halo associated with a late-type $\mathrm{L}^{*}$ galaxy. The radial trend derived from lensing is in very good agreement with simulations and demonstrate that tidal stripping is operational with higher efficiency in the central regions as expected

- Dedicated lensing surveys of well defined massive cluster samples can probe in a systematic way the cluster mass distribution at high resolution from galaxy scales out to the virial radius using both weak and strong lensing. In particular, we can hope to study the build-up of the mass in clusters as a function of time and disentangle the time scales on which the segregation between the different mass components occurs during the assembly process. One could also envision being able to systematically trace the filamentary structures linking massive clusters that are simply the nodes of the cosmic web seen in numerical simulations of the formation and evolution of structure in the Universe.

- From wide field imaging surveys: mass selected clusters can be identified, but likely the more interesting prospect is the ability to investigate the dark matter mass versus stellar-mass relation and its evolution with time, thus providing useful cosmological constraints on the growth of structure in the Universe, as well as on the underlying geometric cosmological parameters.

- With larger cluster samples, better constraints will be obtained on density profile slopes in the inner and outer regions of clusters thus permitting to robustly test theoretical predictions of the $\Lambda \mathrm{CDM}$ model.

- Using numerous multiple images with measured redshifts in a number of massive clusters, we should be able to provide geometrical constraints of Dark Energy in a complementary way to other cosmological probes.

- Measuring the time delays of temporally variable phenomena such as Supernovae or AGN when observed behind well-known massive lensing clusters, will lead to 
measurement of the Hubble parameter $H(z)$, in a similar way as multiple quasars behind galaxies, but with much improved accuracy. However, in order to have a time delay of a limited number of years, these transients event must be located close to the critical lines. While the likelihood of detecting multiple images of such transient phenomena is extremely low, it is not insignificant provided there is a steady increase in the volume of the Universe probed with time.

- Finally, massive cluster lenses will always be the unique places to probe the highredshift Universe, as they offer enhanced sensitivities at all wavelengths and enable mapping the detailed morphology and physical properties of the most distant galaxies in the Universe.

In the near future lensing observations will likely be geared toward optical and near-infrared imaging exploiting the next generation of ground based experiments (DES, LSST, TMT, E-ELT), and spaced based observatories (JWST, EUCLID/WFIRST).

However, in the long run, it is not too unreasonable to think that cluster lensing observations may be well conducted in the radio domain. In such an event we foresee up to an order of magnitude improvement in lensing measurement that will result from combining information on galaxy shapes with velocity field data (Blain 2002; Morales 2006). Such preliminary developments will certainly come first with ALMA, but only centimeter radio interferometers such as SKA (Square Kilometer Array) will allow the exploration of these techniques on cosmological scales.

Acknowledgements The phenomenon of gravitational lensing is one of the most beautiful predictions of Einstein's Theory of General Relativity. We feel very privileged to have had the opportunity to work and contribute to this area. In particular, during and since the start of our scientific collaboration, our goal has been to inter-weave the observational and theoretical aspects of lensing by clusters to enable the exploitation of the potency of this technique. Much of the work reviewed here is heavily based and biased toward recent results, those obtained since JPK's PhD thesis on cluster lenses in 1993; and PN's work on various theoretical aspects of cluster lenses during and since her $\mathrm{PhD}$ in 1999. This review consolidates the rapid progress made thus far and provides a snap shot of this exciting field as it stands today. We would like to thank our many colleagues for the interesting work and fruitful discussions at various conferences, workshops and meetings over the past decade that have provided us a better understanding of our Universe and have transformed cluster lenses into a powerful cosmological tool. JPK acknowledges support from CNRS. PN acknowledges the John Simon Guggenheim Foundation for a Guggenheim Fellowship and the Rockefeller Foundation for a residency at the Bellagio Rockefeller Center during the tenure of which this review was completed. She would also like to thank the Institute for Theory and Computation at Harvard University for hosting her during her fellowship year.

Open Access This article is distributed under the terms of the Creative Commons Attribution Noncommercial License which permits any noncommercial use, distribution, and reproduction in any medium, provided the original author(s) and source are credited.

\section{Appendix: Parametric mass distributions used to model clusters}

Parametric profiles have been extremely successful in modeling cluster mass distributions derived from observed lensing data. A key advantage of parametric models is their flexibility, as they can be used to probe the granularity of the mass distribution on a range of spatial scales. For the case of clusters, this enables combining strong and weak lensing data that derive from different regions of clusters in an optimal fashion. 
Below we outline the lensing properties of three most commonly used mass distributions: the circular Singular Isothermal Sphere [SIS]; the truncated isothermal mass distribution with a core that can be easily extended to the elliptical case [PIEMD] and the Navarro-Frenk-White [NFW] profile. While most mass distributions can be generalized to the elliptical case, there are not always simple and convenient analytic expressions available for lensing quantities as readily as for the PIEMD model. The availability of analytic expressions for the surface mass density, shear and magnification have made the PIEMD a popular choice for modeling lensing clusters.

\section{A.1 The singular isothermal sphere}

The primary motivation for the circular singular isothermal sphere (SIS) profile derives from the good fit that it provides to the observed approximately flat rotation curves of disk galaxies. Flat rotation curves can be reproduced with a model density profile that scales as $\rho \propto r^{-2}$. Such a profile with a constant velocity dispersion as a function of radius appears to provide a good fit to cluster scale halo lenses as well (see e.g. Binney and Tremaine 1987 for more details). The projected surface mass density of the SIS is given by

$$
\Sigma(R)=\frac{\sigma_{v}^{2}}{2 G R},
$$

where $R$ is the distance from the center of the lens in the projected lens plane and where $\sigma_{v}$ is the one-dimensional velocity dispersion of 'particles' that trace the gravitational potential of the mass distribution. The dimensionless surface mass density or convergence is defined in the usual way in units of the critical surface density. For the case of the SIS we have

$$
\kappa(\theta)=\frac{\theta_{\mathrm{E}}}{2 \theta} ; \quad \gamma(\theta)=\frac{\theta_{\mathrm{E}}}{2 \theta},
$$

where $\theta=R / D_{\mathrm{OL}}$ is the angular distance from lens center in the sky plane and where $\theta_{\mathrm{E}}$ is the Einstein deflection angle, defined as

$$
\theta_{\mathrm{E}}=4 \pi\left(\frac{\sigma_{v}}{c}\right)^{2} \frac{D_{\mathrm{LS}}}{D_{\mathrm{OS}}} .
$$

Lensing properties of SIS lens model in a nutshell:

- The magnification and the shear are of the same magnitude; $\kappa=\gamma$ and evaluated at the Einstein radius $\kappa=\gamma=\frac{1}{2}$.

- The tangential critical line is the Einstein ring, and the radial critical line is reduced to the central point.

- The central mass density is infinite, and the total mass is also infinite.

\section{A.2 Truncated isothermal distribution with a core}

Although the SIS is the simplest mass distribution, it is unphysical as it has an infinite central density, an infinite total mass, and therefore cannot adequately match true 
mass distributions. More complex mass distributions have therefore been developed to provide more realistic fits to observed clusters. The Truncated Isothermal Distribution with a Core, which has finite mass and a finite central density is quite popular as a lensing model, and one that we have used extensively and successfully in modeling cluster lenses.

The density distribution for this model is given by

$$
\Sigma(R)=\frac{\Sigma_{0} R_{0} R_{\mathrm{t}}}{R_{\mathrm{t}}-R_{0}}\left(\frac{1}{\sqrt{R_{0}^{2}+R^{2}}}-\frac{1}{\sqrt{R_{\mathrm{t}}^{2}+R^{2}}}\right),
$$

with a model core radius $R_{0}$ and a truncation radius $R_{\mathrm{t}} \gg R_{0}$.

The useful feature of this model, is the ability to reproduce a large range of mass distributions from cluster scales to galaxy scales by varying only the ratio $\eta$, which is defined as $\eta=R_{\mathrm{t}} / R_{0}$. There also exists a simple relation between the truncation radius of the mass distribution and the effective radius $R_{\mathrm{e}}$ of the light distribution for the case of elliptical galaxies:

$$
R_{\mathrm{t}} \sim \frac{4}{3} R_{\mathrm{e}}
$$

Furthermore, this simple circular model can be easily generalized to the elliptical case (Kassiola and Kovner 1993; Kneib et al. 1996) by re-defining the radial coordinate $R$ as follows:

$$
R^{2}=\left(\frac{x^{2}}{(1+\epsilon)^{2}}+\frac{y^{2}}{(1-\epsilon)^{2}}\right) ; \quad \epsilon=\frac{a-b}{a+b} .
$$

Interestingly, all the lensing quantities can be expressed analytically (although using complex numbers) and the expressions for the same were first derived in Kassiola and Kovner (1993).

The mass enclosed within radius $R$ for the model is given by

$$
M(R)=\frac{2 \pi \Sigma_{0} R_{0} R_{\mathrm{t}}}{R_{\mathrm{t}}-R_{0}}\left[\sqrt{R_{0}^{2}+R^{2}}-\sqrt{R_{\mathrm{t}}^{2}+R^{2}}+\left(R_{\mathrm{t}}-R_{0}\right)\right],
$$

and the total mass, which is finite, is

$$
M_{\infty}=2 \pi \Sigma_{0} R_{0} R_{\mathrm{t}}
$$

Calculating $\kappa, \gamma$ and $g$, we have

$$
\kappa(R)=\kappa_{0} \frac{R_{0}}{\left(1-R_{0} / R_{\mathrm{t}}\right)}\left(\frac{1}{\sqrt{\left(R_{0}^{2}+R^{2}\right)}}-\frac{1}{\sqrt{\left(R_{\mathrm{t}}^{2}+R^{2}\right)}}\right),
$$

with

$$
2 \kappa_{0}=\Sigma_{0} \frac{4 \pi G}{c^{2}} \frac{D_{\mathrm{LS}} D_{\mathrm{OL}}}{D_{\mathrm{OS}}}
$$


where $D_{\mathrm{LS}}, D_{\mathrm{OS}}$ and $D_{\mathrm{OL}}$ are, respectively, the lens-source, observer-source and observer-lens angular diameter distances.

To obtain the reduced shear $g(R)$, given the magnification $\kappa(R)$, we solve Laplace's equation for the projected potential $\varphi$, and evaluate the components of the amplification matrix following which we can proceed to solve directly for $\gamma(R)$, and then $g(R)$.

$$
\begin{aligned}
\varphi= & 2 \kappa_{0}\left[\sqrt{R_{0}^{2}+R^{2}}-\sqrt{R_{\mathrm{t}}^{2}+R^{2}}+\left(R_{0}-R_{\mathrm{t}}\right) \ln R\right. \\
& \left.-R_{0} \ln \left[R_{0}^{2}+R_{0} \sqrt{R_{0}^{2}+R^{2}}\right]+R_{\mathrm{t}} \ln \left[R_{\mathrm{t}}^{2}+R_{\mathrm{t}} \sqrt{R_{\mathrm{t}}^{2}+R^{2}}\right]\right] .
\end{aligned}
$$

We can then derive the shear $\gamma(R)$ :

$$
\begin{array}{r}
\gamma(R)=\kappa_{0}\left[-\frac{1}{\sqrt{R^{2}+R_{0}^{2}}}+\frac{2}{R^{2}}\left(\sqrt{R^{2}+R_{0}^{2}}-R_{0}\right)\right. \\
\left.+\frac{1}{\sqrt{R^{2}+R_{\mathrm{t}}^{2}}}-\frac{2}{R^{2}}\left(\sqrt{R^{2}+R_{\mathrm{t}}^{2}}-R_{\mathrm{t}}\right)\right] .
\end{array}
$$

Scaling this relation by $R_{\mathrm{t}}$ gives for $R_{0}<R<R_{\mathrm{t}}$

$$
\gamma\left(R / R_{\mathrm{t}}\right) \propto \frac{\Sigma_{0}}{\eta-1} \frac{R_{\mathrm{t}}}{R} \sim \frac{\sigma^{2}}{R},
$$

where $\sigma$ is the velocity dispersion (note this is similar to the SIS case).

At larger radius, for $R_{0}<R_{\mathrm{t}}<R$

$$
\gamma\left(R / r_{\mathrm{t}}\right) \propto \frac{\Sigma_{0}}{\eta} \frac{R_{\mathrm{t}}^{2}}{R^{2}} \sim \frac{M_{\mathrm{tot}}}{R^{2}},
$$

where $M_{\mathrm{tot}}$ is the total mass. In the limit that $R \gg R_{\mathrm{t}}$, we have

$$
\gamma(R)=\frac{3 \kappa_{0}}{2 R^{3}}\left[R_{0}^{2}-R_{\mathrm{t}}^{2}\right]+\frac{2 \kappa_{0}}{R^{2}}\left[R_{\mathrm{t}}-R_{0}\right] .
$$

Lensing properties of the truncated isothermal distribution with a core in a nutshell:

- $\kappa \neq \gamma$.

- The tangential critical line once again corresponds to the Einstein ring, and the radial critical line is a circle interior to the Einstein ring.

- The central mass density is finite, and the total mass is also finite.

\section{A.3 The Navarro-Frenk-White model}

Although the truncated isothermal distribution with a core is very popular, it has never been fitted to the results of numerical simulations, in contrast to the universal 
"NFW" density profile (Navarro et al. 1997). In simulations of structure formation and evolution in the Universe, the NFW profile was found to be a good fit for a wide range of dark matter halo masses from $10^{9}-10^{15} \mathrm{M}_{\odot}$. The spherical NFW density profile has the following form:

$$
\rho(r)=\frac{\rho_{\mathrm{s}}}{\left(r / r_{\mathrm{s}}\right)\left(1+r / r_{\mathrm{s}}\right)^{2}},
$$

where $\rho_{\mathrm{S}}$ and $r_{\mathrm{s}}$ are free parameters. It is often convenient to characterize the profile with the concentration parameter, $c_{\mathrm{vir}}=r_{\mathrm{vir}} / r_{\mathrm{s}}$ where $r_{\mathrm{vir}}$ is the virial radius. By integrating the profile out to $r_{\mathrm{vir}}$ and using $m_{\mathrm{vir}}=200 \rho_{\mathrm{c}}(z) 4 \pi r_{\mathrm{vir}}^{3} / 3$, where $m_{\mathrm{vir}}$ is defined to be the virial mass and $\rho_{\mathrm{c}}$ is the critical density of the universe, the concentration parameter can be related to $\rho_{\mathrm{s}}$.

We now proceed to calculate the lensing properties of the NFW profile (more details can be found in Wright and Brainerd 2000). In the thin lens approximation, $z$ is defined as the optical axis and $\Phi(R, z)$ the three-dimensional Newtonian gravitational potential, where $r=\sqrt{R^{2}+z^{2}}$. The reduced two-dimensional lens potential in the plane of the sky is given by

$$
\varphi(\vec{\theta})=\frac{2}{c^{2}} \frac{D_{\mathrm{LS}}}{D_{\mathrm{OL}} D_{\mathrm{OS}}} \int_{-\infty}^{+\infty} \Phi\left(D_{\mathrm{OL}} \theta, z\right) d z,
$$

where $\vec{\theta}=\left(\theta_{1}, \theta_{2}\right)$ is the angular position in the image plane.

For convenience we introduce the dimensionless radial coordinates $\vec{x}=\left(x_{1}, x_{2}\right)=$ $\vec{R} / r_{\mathrm{s}}=\vec{\theta} / \theta_{\mathrm{s}}$ where $\theta_{\mathrm{s}}=r_{\mathrm{s}} / D_{\mathrm{OL}}$. In the case of an axially symmetric lens, the relations become simpler, as the position vector can be replaced by its norm. The surface mass density then becomes

$$
\Sigma(x)=\int_{-\infty}^{+\infty} \rho\left(r_{\mathrm{s}} x, z\right) d z=2 \rho_{\mathrm{c}} r_{\mathrm{s}} F(x),
$$

with

$$
F(x)= \begin{cases}\frac{1}{x^{2}-1}\left(1-\frac{1}{\sqrt{1-x^{2}}} \operatorname{arcch} \frac{1}{x}\right) & (x<1), \\ \frac{1}{3} & (x=1), \\ \frac{1}{x^{2}-1}\left(1-\frac{1}{\sqrt{x^{2}-1}} \arccos \frac{1}{x}\right) & (x>1)\end{cases}
$$

and the mean surface density inside the dimensionless radius $x$ is

$$
\bar{\Sigma}(x)=\frac{1}{\pi x^{2}} \int_{0}^{x} 2 \pi x \Sigma(x) d x=4 \rho_{\mathrm{c}} r_{\mathrm{s}} \frac{g(x)}{x^{2}},
$$

with

$$
g(x)= \begin{cases}\ln \frac{x}{2}+\frac{1}{\sqrt{1-x^{2}}} \operatorname{arcch} \frac{1}{x} & (x<1), \\ 1+\ln \frac{1}{2} & (x=1), \\ \ln \frac{x}{2}+\frac{1}{\sqrt{x^{2}-1}} \arccos \frac{1}{x} & (x>1) .\end{cases}
$$


The lensing functions $\vec{\alpha}, \kappa$ and $\gamma$ also have simple expressions:

$$
\left\{\begin{array}{l}
\vec{\alpha}(x)=\theta \frac{\bar{\Sigma}(x)}{\Sigma_{\text {crit }}}=4 \kappa_{\mathrm{s}} \frac{\theta}{x^{2}} g(x) \vec{e}_{x}, \\
\kappa(x)=\frac{\Sigma(x)}{\Sigma_{\text {crit }}}=2 \kappa_{\mathrm{s}} F(x), \\
\gamma(x)=\frac{\bar{\Sigma}(x)-\Sigma(x)}{\Sigma_{\text {crit }}}=2 \kappa_{\mathrm{s}}\left(\frac{2 g(x)}{x^{2}}-F(x)\right)
\end{array}\right.
$$

with $\kappa_{\mathrm{s}}=\rho_{\mathrm{c}} r_{\mathrm{s}} \Sigma_{\text {crit }}^{-1}$. Noting $\vec{\nabla}_{\vec{x}} \alpha(x)=\left(\partial_{x_{1}} \alpha, \partial_{x_{2}} \alpha\right)$ and $\phi=\arctan \left(x_{2} / x_{1}\right)$, we obtain some useful relations for the following that hold for any circular mass distribution (Golse and Kneib 2002):

$$
\left\{\begin{array}{l}
\kappa(x)=\frac{1}{2 \theta_{\mathrm{s}}}\left(\frac{\alpha(x)}{x}+\frac{\partial_{x_{1}} \alpha(\vec{x})}{\cos \phi}\right) 0, \\
\gamma(x)=\frac{1}{2 \theta_{\mathrm{s}}}\left(\frac{\alpha(x)}{x}-\frac{\partial_{x_{1}} \alpha(\vec{x})}{\cos \phi}\right), \\
\frac{\partial_{x_{1}} \alpha(\vec{x})}{\cos \phi}=\frac{\partial_{x_{2}} \alpha(\vec{x})}{\sin \phi}, \\
\kappa(x)+\gamma(x)=\frac{\alpha(x)}{\theta_{\mathrm{s}} x} .
\end{array}\right.
$$

By integrating the deflection angle we obtain the lens potential $\varphi(x)$ :

$$
\varphi(x)=2 \kappa_{\mathrm{s}} \theta_{\mathrm{s}}^{2} h(x)
$$

where

$$
h(x)= \begin{cases}\ln ^{2} \frac{x}{2}-\operatorname{arcch}^{2} \frac{1}{x} & (x<1) \\ \ln ^{2} \frac{x}{2}+\arccos ^{2} \frac{1}{x} & (x \geq 1) .\end{cases}
$$

The velocity dispersion $\sigma(r)$ of this potential, computed with the Jeans equation for an isotropic velocity distribution, gives an unrealistic central velocity dispersion $\sigma(0)=0$. In order to compare the pseudo-elliptical NFW potential with other potentials, we define a scaling parameter $v_{\mathrm{c}}$ (characteristic velocity) in terms of the parameters of the NFW profile as follows:

$$
v_{\mathrm{c}}^{2}=\frac{8}{3} \mathrm{G} r_{\mathrm{s}}^{2} \rho_{\mathrm{c}}
$$

Using the value of the critical density for closure of the Universe $\rho_{\text {crit }}=3 H_{0}^{2} / 8 \pi G$, we find

$$
\frac{\rho_{\mathrm{c}}}{\rho_{\text {crit }}}=\frac{v_{\mathrm{c}}^{2}}{H_{0}^{2} r_{\mathrm{s}}^{2}}=1.810^{3} h^{-2} \times\left(\frac{r_{\mathrm{s}}}{150 \mathrm{kpc}}\right)^{-2}\left(\frac{v_{\mathrm{c}}}{2000 \mathrm{~km} \mathrm{~s}^{-1}}\right)^{2} .
$$

Lensing properties of the NFW model in a nutshell: 


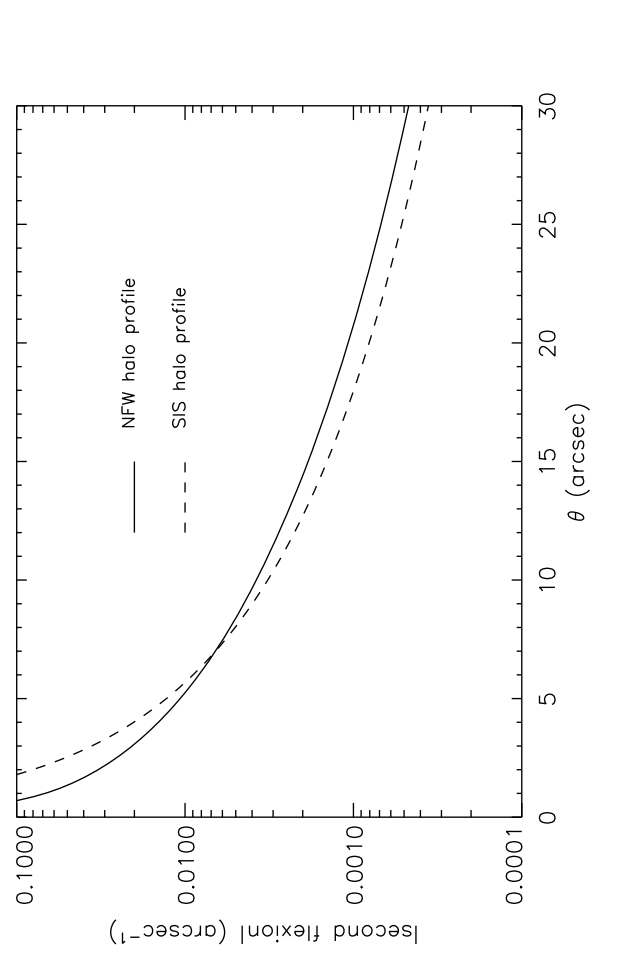

눙

농

苯

号

气 हี

$\because \bar{\Xi}$

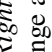

흔

थे

II

急

난.

핼

D.

$\stackrel{\sigma}{=}$

T

ป ข

음

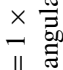

\&ิ ะ

इ

पे

오 ․․

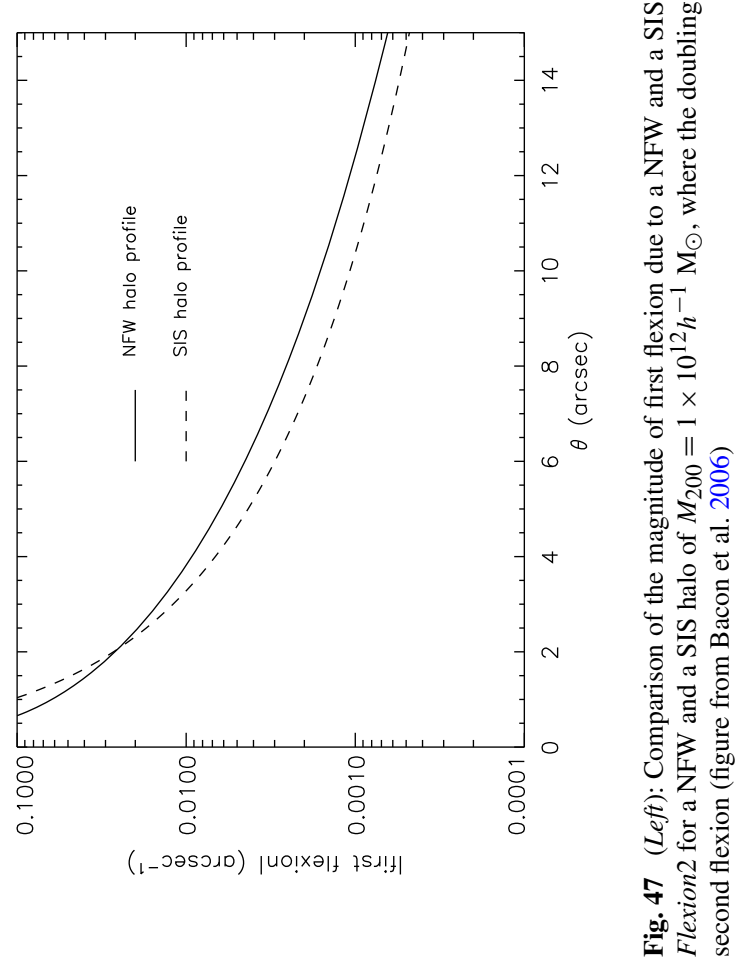


- $\kappa \neq \gamma$.

- The tangential critical line is the Einstein ring radius, and the radial critical line is a circle interior to the Einstein ring.

- The central mass density is infinite, and the total mass is also infinite (however, only the virial mass is of interest and that is calculable), and the central velocity dispersion is vanishing at the center.

\section{A.4 Flexion for the singular isothermal sphere}

For example, consider a Schwarzschild lens: the first flexion is by definition zero everywhere except at the origin, as the gradient of the convergence is zero everywhere except at the origin. However, there is certainly "arciness" generated by such a lens; this is described by the second flexion. We will provide explicit expressions for the first and second flexion generated by the simple mass distribution of a singular isothermal sphere. The flexion caused by the SIS at an angular vector displacement, $\theta$, from the lens center on the sky plane is thus simply

$$
\mathcal{F}=-\left[\frac{\theta_{\mathrm{E}}}{2 \theta^{2}}\right] e^{i \phi},
$$

where $\phi$ is the position angle around the lens, and in this case also gives the direction of the flexion. The first flexion $\mathcal{F}$ for this profile is therefore circularly symmetric and (expressed as a vector) directed radially inwards toward the lens center, as would be expected.

Similarly, the second flexion $\mathcal{G}$ is

$$
\mathcal{G}=\frac{3 \theta_{\mathrm{E}}}{2 \theta^{2}} e^{3 i \phi} .
$$

This has a larger maximum amplitude than the first flexion for this lens profile, fades off with the same power law index away from the lens, and oscillates around the lens as a spin-3 quantity rather than a spin-1 quantity. For the explicit derivation of the flexion for more complicated density profiles, namely the softened isothermal sphere and the cosmologically motivated Navarro-Frenk-White profile, see Fig. 47 for a comparison as well as Bacon et al. (2006).

\section{References}

Abdelsalam H et al (1998a) Astron J 116:1541

Abdelsalam HM, Saha P, Williams LLR (1998b) Mon Not R Astron Soc 294:734

Ade PAR et al (2011) Astron Astrophys (in press). arXiv:1101.2024

Aihara H et al (2011) Astrophys J Suppl Ser 195:26

Allen SW (1998) Mon Not R Astron Soc 296:392

Allen SW, Schmidt RW, Fabian AC (2001a) Mon Not R Astron Soc 328:L37

Allen SW, Schmidt RW, Fabian AC (2002) Mon Not R Astron Soc 334:L11

Allen SW, Schmidt RW, Ebeling H, Fabian AC, van Speybroeck L (2004) Mon Not R Astron Soc 353:457

Altieri B et al (1999) Astron Astrophys 343:L65

Altieri B et al (2010) Astron Astrophys 518:17

Amanullah R et al (2010) Astrophys J 716:712 
Amanullah R, Goobar A, Clément B et al. (2011). arXiv:1109.4740

Andersson K, Madejski G (2004) Astrophys J 607:190

Avila-Reese V, Colin P, Piccinelli G, Firmani C (2003) Astrophys J 598:36

Bacon D, Refregier A, Ellis RS (2000) Mon Not R Astron Soc 318:625

Bacon DJ et al (2005) Mon Not R Astron Soc 363:723

Bacon DJ, Goldberg DM, Rowe BTP, Taylor AN (2006) Mon Not R Astron Soc 365:414

Bahcall J, Sarazin C (1977) Astrophys J 213:99

Bardeau S, Kneib J-P, Czoske O, Soucail G, Smail I, Ebeling H, Smith GP (2005) Astron Astrophys 434:433

Bardeau S, Soucail G, Kneib J-P, Czoske O, Ebeling H, Hudelot P, Smail I, Smith G (2007) Astron Astrophys 470:449

Barger AJ et al (2002) Astron J 124:1839

Barnothy JM (1965) Astrophys J 70:666

Bartelmann M (1995) Astron Astrophys 303:643

Bartelmann M et al (1996) Astrophys J 446:L115

Bartelmann M, Huss A, Colberg J, Jenkins A, Pearce FR (1998) Astron Astrophys 330:1

Bartelmann M, Meneghetti M, Perrotta F, Baccigalupi C, Moscardini L (2003) Astron Astrophys 409:449

Bautz LP, Morgan WW (1970) Astrophys J 162:L149

Bayliss M, Hennawi J, Gladders M, Koester B, Sharon K, Dahle H, Oguri M (2011) Astrophys J Suppl Ser 193:8

Berge J et al (2008) Mon Not R Astron Soc 385:695

Berta S et al (2010) Astron Astrophys 518:30

Bertin E, Arnouts S (1996) Astron Astrophys Suppl Ser 117:393

Bezecourt J et al (1999) Astron Astrophys 347:21

Binney J, Tremaine S (1987) Galactic dynamics. Princeton University Press, Princeton

Blain AW (1997) Mon Not R Astron Soc 290:553

Blain AW (1998) MNPvAS 295:92

Blain A (2002) Astrophys J Lett 570:51

Blain A, Smail I, Ivison R, Kneib J-P, Frayer D (2002) Phys Res 369:111

Blandford RD, Narayan R (1986) Astrophys J 310:568

Blandford RD, Kochanek C, Kovner I, Narayan R (1989) Science 245:824

Blumenthal GR, Faber SM, Primack J, Rees MJ (1984) Nature 311:517

Blumenthal GR, Faber SM, Flores R, Primack J (1986) Astrophys J 301:27

Boehringer H et al (2004) Astron Astrophys 425:367

Bonnet H, Mellier Y, Fort B (1994) Astrophys J 427:83

Boone F, Schaerer D, Pelló R, Combes F, Richard J (2007) Astron Astrophys 475:513

Borys C et al (2004) Mon Not R Astron Soc 352:759

Borys C et al (2005) ESASP 577:275

Bradač M (2009) Nucl Phys B, Proc Suppl 194:17

Bradač M, Lombardi M, Schneider P (2004) Astron Astrophys 424:13

Bradač M, Schneider P, Lombardi M, Erben T (2005) Astron Astrophys 437:49

Bradač M et al (2006) Astrophys J 652:937

Bradač M et al (2008) Astrophys J 687:959

Bradley LD et al (2008) Astrophys J 678:647

Bradley LD et al. (2011) Preprint. arXiv:1104.2035

Brainerd T, Blandford R, Smail I (1996) Astrophys J 466:623

Bridle S et al (1998) Mon Not R Astron Soc 299:895

Bridle S et al (2002) In: Natarajan P (ed) The shapes of galaxies and their dark halos, Yale cosmology workshop. World Scientific, Singapore, p 38

Bridle S et al (2010) Mon Not R Astron Soc 405:2044

Broadhurst T, Benitez N, Coe D et al (2005) Astrophys J 621:53

Broadhurst T, Umetsu K, Medezinski E, Oguri M, Rephaeli Y (2008) Astrophys J 685:L9

Buote D, Canizares C (1996) Astrophys J 457:565

Burke WL (1981) Astrophys J Lett 244:1

Cabanac R et al (2007) Astron Astrophys 461:813

Campusano LE, Pelló R, Kneib J-P, Le Borgne J-F, Fort B, Ellis R, Mellier Y, Smail I (2001) Astron Astrophys 378:394

Cavaliere A, Fusco-Femiano R (1978) Astron Astrophys 70:677 
Chang CL et al (2009) AIPC 1185:475

Chapman S, Lewis G, Scott D, Borys C, Richards E (2002) Astrophys J 570:557

Chapman S, Blain A, Smail I, Ivison R (2005) Astrophys J 622:772

Chary R, Stern D, Eisenhardt P (2005) Astrophys J Lett 635:5

Clement B et al (2011) Preprint. arXiv:1105.4072

Clowe D, Schneider P (2001) Astron Astrophys 379:384

Clowe D, Schneider P (2002) Astron Astrophys 395:385

Clowe D, Luppino GA, Kaiser N, Henry JP, Gioia IM (1998) Astrophys J Lett 497:L61

Clowe D, Gonzalez A, Markevitch M (2004a) Astrophys J 604:596

Clowe D, De Lucia G, King L (2004b) Mon Not R Astron Soc 350:1038

Clowe D, Bradač M, Gonzalez AH, Markevitch M, Randall S, Jones C, Zaritsky D (2006) Astrophys J 648:L109

Coe D, Fuselier E, Benitez N, Broadhurst T, Frye B, Ford H (2008) Astrophys J 681:814

Coe D et al (2010) Astrophys J 723:1678

Coia D et al (2005a) Astron Astrophys 431:433

Coia D et al (2005b) Astron Astrophys 430:59

Collins CA, Burke DJ, Romer AK, Sharples RM, Nichol RC (1997) Astrophys J 479:L117

Comerford J, Natarajan P (2007) Mon Not R Astron Soc 379:190

Cooray AR (1999a) Astron Astrophys 341:653

Cooray AR (1999b) Mon Not R Astron Soc 305:412

Corless V, King L, Clowe D (2009) Mon Not R Astron Soc 393:1235

Cowie LL, Barger AJ, Kneib J-P (2002) Astron J 123:2197

Crittenden R, Natarajan P, Pen U, Theuns T (2001) Astrophys J 559:552

Crittenden R, Natarajan P, Pen U, Theuns T (2002) Astrophys J 568:20

Cypriano ES, Sodré LJ, Campusano LE, Kneib J-P, Giovanelli R, Haynes MP, Dale DA, Hardy E (2001) Astron J 121:10

Czoske O et al (2001) Astron Astrophys 372:391

Daddi E et al (2000) Astron Astrophys 361:535

Dahle H, Kaiser N, Irgens RJ, Lilje PB, Maddox SJ (2002) Astrophys J Suppl Ser 139:313

Dalal N, Holder G, Hennawi J (2004) Astrophys J 609:50

Dalal N, Hennawi JF, Bode P (2005) Astrophys J 622:99

Dalal N, Dore O, Huterer D, Shirokov A (2008) Phys Rev D 77:123514

D'Aloisio A, Natarajan P (2011a) Mon Not R Astron Soc 411:1628

D'Aloisio A, Natarajan P (2011b) Mon Not R Astron Soc (in press). arXiv: 1102.5097

Dawson KS et al (2009) Astron J 138:1271

Deb S, Goldberg D, Ramdass V (2008) Astrophys J 687:39

Diego J, Protopapas P, Sandvik H, Tegmark M (2005a) Mon Not R Astron Soc 360:477

Diego J, Sandvik H, Protopapas P, Tegmark M, Benitez N, Broadhurst T (2005b) Mon Not R Astron Soc $362: 1247$

Diego J, Tegmark M, Protopapas P, Sandvik HB (2007) Mon Not R Astron Soc 375:958

Diehl HT et al (2009) Astrophys J 707:686

Dressler A (1980) Astrophys J 236:351

Dye S, Warren S (2005) Astrophys J 623:31

Ebbels T et al (1996) Mon Not R Astron Soc 281:L75

Ebbels T et al (1998) Mon Not R Astron Soc 295:75

Ebeling H et al (1996) Mon Not R Astron Soc 281:799

Ebeling H et al (1998) Mon Not R Astron Soc 301:881

Ebeling H et al (2000) Astrophys J 534:133

Ebeling H et al (2001) Astrophys J 553:668

Ebeling H, Barrett E, Donovan D (2004) Astrophys J 609:L49

Ebeling H et al (2009) Mon Not R Astron Soc 395:1213

Efstathiou G, Moody S, Peacock JA et al (2002) Mon Not R Astron Soc 330:L29

Egami E et al (2005) Astrophys J Lett 618:5

Egami E et al (2010) Astron Astrophys 518:12

Eisenstein D et al (2005) Astrophys J 633:560

Ellis R, Santos MR, Kneib J-P, Kuijken K (2001) Astrophys J Lett 560:L119

Er X, Li G, Schneider P (2011) Astron Astrophys (in press). arXiv:1008.3088

Erdl H, Schneider P (1993) Astron Astrophys 268:453 
Estrada J et al (2007) Astrophys J 660:1176

Etherington I (1933) Philos Mag 15:761

Fahlman G, Kaiser N, Squires G, Woods D (1994) Astrophys J 437:56

Falco EE, Gorenstein MV, Shapiro II (1985) Astrophys J Lett 289:L1

Fassbender R, Böhringer H, Santos J, Lamer G, Mullis C, Schuecker P, Schwope A, Rosati P (2008) In: Conf proc relativistic astrophysics legacy and cosmology, ESO astrophysics symposia, p 307

Fedeli C, Bartelmann M (2007) Astron Astrophys 461:49

Fedeli C, Bartelmann M, Meneghetti M, Moscardini L (2008) Astron Astrophys 486:35

Fedeli C, Meneghetti M, Gottlober S, Yepes G (2010) Astron Astrophys 519:A91

Fischer P, Tyson JA (1997) Astron J 114:14

Fort B et al (1992) Astrophys J 399:L125

Franx M et al (1997) Astrophys J 486:L75

Frayer D, Ivison R, Scoville N, Yun M, Smail I, Blain A, Kneib J-P (1998) Astrophys J 506:7

Frye B, Broadhurst T (1998) Astrophys J Lett 499:L115

Fu H, Stockton A (2008) Astrophys J 677:79

Gal-Yam A, Maoz D, Sharon K (2002) Mon Not R Astron Soc 332:37

Gautret L, Fort B, Mellier Y (2000) Astron Astrophys 353:10

Gavazzi R (2005) Astron Astrophys 443:793

Gavazzi R, Soucail G (2007) Astron Astrophys 462:459

Gavazzi R, Fort B, Mellier Y, Pelló R, Dantel-Fort M (2003) Astron Astrophys 403:11

Geiger B, Schneider P (1999) Mon Not R Astron Soc 302:118

Gilbank D, Gladders M, Yee H, Hsieh B (2011) Astron J 141:94

Gilmore J, Natarajan P (2009) Mon Not R Astron Soc 396:354

Gioia IM, Shaya EJ, Le Fevre O, Falco EE, Luppino GA, Hammer F (1998) Astrophys J 497:573

Gladders M (2002) PhD thesis, University of Toronto, Toronto, Canada

Gladders M, Yee H (2005) Astrophys J Suppl Ser 157:1

Gladders MD, Yee HKC, Ellingson E (2002) Astron J 123:1

Gladders M, Hoekstra H, Yee HK, Hall PB, Barrientos L (2003) Astrophys J 593:48

Gnedin O, Kravtsov AV, Klypin AA, Nagai D (2004) Astrophys J 616:16

Goldberg D, Bacon D (2005) Astrophys J 619:741

Goldberg D, Natarajan P (2002) Astrophys J 564:65

Golse G, Kneib J-P (2002) Astron Astrophys 390:821

Golse G, Kneib J-P, Soucail G (2002) Astron Astrophys 387:788

Golse G, Kneib J-P, Soucail G (2004) Astron Astrophys 417:33

Goobar A et al (2009) Astron Astrophys 507:71

Gruen D, Bernstein GM, Lam TY, Seitz S (2011) Mon Not R Astron Soc 416:1392

Gull S, Northover KJE (1976) Nature 283:572

Gunnarsson C, Goobar A (2003) Astron Astrophys 405:859

Halkola A, Seitz S, Pannella M (2006) Mon Not R Astron Soc 372:1425

Hamana T, Takada M, Yoshida N (2004) Mon Not R Astron Soc 350:893

Hand N et al (2011) Astrophys J 736:39

Hennawi J, Dalal N, Bode P, Ostriker J (2007) Astrophys J 654:714

Hennawi J et al (2008) Astron J 135:664

Hetterscheidt M, Erben T, Schneider P (2005) Baryons in dark matter halos

Heymans C et al (2006) Mon Not R Astron Soc 371:60

Hincks AD et al (2010) Astrophys J Suppl Ser 191:423

Hoekstra H (2007) Mon Not R Astron Soc 379:317

Hoekstra H, Franx M, Kuijken K (2000) Astrophys J 532:88

Hoekstra H, Franx M, Kuijken K, van Dokkum PG (2002) Mon Not R Astron Soc 333:911

Hoekstra H et al (2006) Astrophys J 647:116

Hoessl JG, Gunn JE, Thuan TX (1980) Astrophys J 241:486

Horesh A, Ofek E, Maoz D, Bartelmann M, Meneghetti M, Rix H-W (2005) Astrophys J 633:768

Horesh A, Maoz D, Ebeling H, Seidel G, Bartelmann M (2010) Mon Not R Astron Soc 406:1318

Hu E et al (2002) Astrophys J 586:L75

Hubble EP (1929) Leafl, Astron Soc Pac 1:93

Hubble E (1931) Astrophys J 74:43

Huterer D, White M (2002) Astrophys J Lett 578:L95

Irgens RJ, Lilje PB, Dahle H, Maddox SJ (2002) Astrophys J 579:227 
Ivison R et al (1998) MNPvAS 298:583

Ivison R et al (2002) Mon Not R Astron Soc 337:1

Jauzac A et al. (2011) submitted

Jee MJ, White RL, Benítez N, Ford HC, Blakeslee JP, Rosati P, Demarco R, Illingworth GD (2005) Astrophys J 618:46

Joffre M et al (2000) Astrophys J Lett 534:L131

Jorgensen I, Franx M, Kjaergaard P (1996) Mon Not R Astron Soc 280:167

Jullo E, Kneib J-P (2009) Mon Not R Astron Soc 395:1319

Jullo E, Kneib J-P, Limousin M, Elíasdóttir Á, Marshall PJ, Verdugo T (2007) New J Phys 9:447

Jullo E, Natarajan P, Kneib J-P, D'Alosio A, Limousin M, Richard J, Schimd C (2010) Science 329:924

Kaiser N (1986) Mon Not R Astron Soc 222:323

Kaiser N (1992) Astrophys J 388:272

Kaiser N (1995) Astrophys J Lett 439:L1

Kaiser N, Squires G (1993) Astrophys J 404:441

Kaiser N et al (1995) Astrophys J 449:460

Kaiser N et al (1998) A photometric and weak lensing analysis of the $z=0.42$ supercluster MS0302 +17 . astro-ph/9809268

Kaiser N, Wilson G, Luppino G (2000) Preprint. arXiv:astro-ph/0003338

Kassiola A, Kovner I (1993) Astrophys J 417:450

Kassiola A et al (1992) Astrophys J 400:41. (1997) Astron Astrophys Suppl Ser 122:471

King LJ, Schneider P (2001) Astron Astrophys 369:1

King LJ, Schneider P, Springel V (2001) Astron Astrophys 378:748

King LJ, Clowe DI, Schneider P (2002) Astron Astrophys 383:118

Kitching TD, Miller L, Heymans C, van Waerbeke L, Heavens AF (2008) Mon Not R Astron Soc 390:149

Kitching T, Heavens A, Miller L (2011) Mon Not R Astron Soc 413:2923

Kneib J-P (1993) PhD thesis, Universite Paul Sabatier, Toulouse, France

Kneib J-P, Mellier Y, Fort B, Mathez G (1993) Astron Astrophys 273:367

Kneib J-P, Melnick J, Gopal-Krishna (1994) Astron Astrophys 290:L25

Kneib J-P, Ellis RS, Smail I, Couch WJ, Sharples RM (1996) Astrophys J 471:643

Kneib J-P et al (2003) Astrophys J 598:804

Kneib J-P, Ellis RS, Santos MR, Richard J (2004) Astrophys J 607:697

Kneib J-P, Neri R, Smail I, Blain A, Sheth K, van der Werf P, Knudsen K (2005) Astron Astrophys 434:819

Kneib J-P et al (2011) in preparation

Knudsen K et al (2005) Astrophys J 632:9

Knudsen K, van der Werf P, Kneib J-P (2008) Mon Not R Astron Soc 384:1611

Knudsen K, Kneib J-P, Richard J, Petitpas G, Egami E (2010) Astrophys J 709:210

Kochanek CS (1990) Mon Not R Astron Soc 247:135

Kochanek C, Apostolakis J (1988) Mon Not R Astron Soc 235:1073

Kochanek C et al (1995) Astrophys J 445:549

Kodama T, Smail I, Nakata F, Okamura S, Bower RG (2002) ASPC 268:301

Kolatt TS, Bartelmann M (1998) Mon Not R Astron Soc 296:763

Komatsu E, Spergel DN (2001) Phys Rev D 63:063002

Komatsu E et al (2011) Astrophys J Suppl Ser 192:18

Kovner I, Paczynski B (1988) Astrophys J Lett 335:L9

Kubo JM et al (2009) Astrophys J Lett 702:110

Kuijken K (1999) Astron Astrophys 352:355

Laporte N et al (2011) Astron Astrophys 531:74

Lea S, Silk J, Kellogg E, Murray S (1973) Astrophys J Lett 184:L105

Leauthaud A et al (2010) Astrophys J Lett 709:97

Leauthaud A et al. (2011) Preprint. arXiv:1104.0928

Leir A, van den Bergh S (1977) Astrophys J Suppl Ser 34:381

Lemaître G (1931) Mon Not R Astron Soc 91:483

Lemoine-Busserolle M, Contini T, Pelló R, Le Borgne J-F, Kneib J-P, Lin C (2003) Astron Astrophys 397:839

Lemze D, Barkana R, Broadhurst T, Rephaeli Y (2008) Mon Not R Astron Soc 386:1092

Leonard A, King L (2010) Mon Not R Astron Soc 405:1854

Leonard A, King L, Goldberg D (2011) Mon Not R Astron Soc 413:789

Lewis J, Smail I, Couch W (2000) Astrophys J 528:118 
Li G, Mao S, Jing Y-P, Bartelmann M, Kang X, Meneghetti M (2005) Astrophys J Lett 635:795

Liesenborgs J, de Rijcke S, Dejonghe H, Bekaert P (2007) Mon Not R Astron Soc 380:1729

Limousin M, Kneib J, Natarajan P (2005) Mon Not R Astron Soc 356:309

Limousin M, Kneib J-P, Bardeau S, Natarajan P, Czoske O, Smail EH, Smith G (2007) Astron Astrophys 461:881

Limousin M et al (2008) Astron Astrophys 489:23

Limousin M et al (2009) Astron Astrophys 502:445

Limousin M et al (2010) Astron Astrophys 524:95

Limousin M et al (2011) Astron Astrophys (submitted). arXiv:1109.3301

Link R, Pierce M (1998) Astrophys J 502:63

Loeb A, Mao S (1994) Astrophys J Lett 435:L109

Lombardi M, Bertin G (1998) Astron Astrophys 335:1

Lombardi M et al (2005) Astrophys J 623:42

Lo Verde M, Miller A, Shandera S, Verde L (2008) J Cosmol Astropart Phys 4:14

Luppino GA, Gioia IM (1992) Astron Astrophys 265:L9

Luppino GA, Kaiser N (1997) Astrophys J 475:20

Luppino GA, Gioia IM, Hammer F, Le Fèvre O, Annis JA (1999) Astron Astrophys Suppl Ser 136:117

Lynds R, Petrosian V (1989) Astrophys J 336:L1

Maccio A (2005) Mon Not R Astron Soc 361:1250

Madhavi A, Hoekstra H, Babul A, Henry JP (2008) MNPvAS 384:1567

Mahdavi A, Hoekstra H, Babul A, Balam D, Capak P (2007) Mon Not R Astron Soc 380:437

Mandelbaum R et al (2005) Mon Not R Astron Soc 361:1287

Mandelbaum R, Seljak U, Kauffmann G, Hirata C, Brinkmann J (2006) Mon Not R Astron Soc 368:715

Mandelbaum R, Seljak U, Baldauf T, Smith R (2010) Mon Not R Astron Soc 405:2078

Markevitch M, Gonzalez A, David L, Vikhlinin A, Murray S, Forman W, Jones C, Tucker W (2002) Astrophys J Lett 567:27

Marriage T et al (2011) Astrophys J 732:44

Marrone D et al. (2011) Preprint. arXiv:1107.5115

Marshall P (2003) PhD thesis, University of Cambridge, UK

Marshall P (2006) Mon Not R Astron Soc 372:1289

Marshall P, Hobson M, Gull S, Bridle S (2002) Mon Not R Astron Soc 335:1037

Massey R (2010) Mon Not R Astron Soc 409:109

Massey R et al (2007) Mon Not R Astron Soc 376:13

Matarrese S, Verde L, Jimenez R (2000) Mon Not R Astron Soc 316:283

Maturi M, Bartelmann M, Meneghetti M, Moscardini L (2005) Astron Astrophys 436:37

Mellier Y et al (1993) Astrophys J 407:33

Meneghetti M, Bartelmann M, Moscardini L (2003a) Mon Not R Astron Soc 340:105

Meneghetti M, Bartelmann M, Moscardini L (2003b) Mon Not R Astron Soc 346:67

Meneghetti M, Jain B, Bartelmann M, Dolag K (2005a) Mon Not R Astron Soc 362:1301

Meneghetti M, Bartelmann M, Dolag K, Perrotta F, Baccigalupi C, Moscardini L, Tormen G (2005b) New Astron Rev 49:111

Meneghetti M, Fedeli C, Pace F, Gottloeber S, Yepes G (2010) Astron Astrophys 519:A90

Meneghetti M, Fedeli C, Zitrin A, Bartelmann M, Broadhurst T, Gottloeber S, Moscardini L, Yepes G (2011) Astron Astrophys 530:17

Metcalfe L et al (2003) Astron Astrophys 407:791

Metzler CA, White M, Norman M, Loken C (1999) Astrophys J Lett 520:L9

Metzler CA, White M, Loken C (2001) Astrophys J 547:560

Miller L, Kitching TD, Heymans C, Heavens AF, van Waerbeke L (2007) Mon Not R Astron Soc 382:315

Miralda-Escudé J (1991) Astrophys J 370:1

Miralda-Escudé J (1995) Astrophys J 438:514

Miralda-Escudé J (2002) Astrophys J 564:60

Miralda-Escudé J, Babul A (1995) Astrophys J 449:18

Miralda-Escudé J, Fort B (1993) Astrophys J Lett 417:L5

Moore B et al (1998) Astrophys J 499:L5

Morales M (2006) Astrophys J Lett 650:21

Moran S et al (2006) Astrophys J 641:L97

Morandi A, Pedersen K, Limousin M (2010) Astron Astrophys 527:124

Narayan R, Blandford R, Nityananda R (1984) Nature 310:112 
Narasimha D, Chitre SM (1988) Astrophys J 332:75

Natarajan P, Kneib J-P (1996) Mon Not R Astron Soc 283:1031

Natarajan P, Kneib J-P (1997) Mon Not R Astron Soc 287:833

Natarajan P, Springel V (2004) Astrophys J 617:L13

Natarajan P, Kneib J, Smail I, Ellis RS (1998) Astrophys J 499:600

Natarajan P, Kneib J, Smail I (2002a) Astrophys J Lett 580:L11

Natarajan P, Loeb A, Kneib J-P, Smail I (2002b) Astrophys J Lett 580:L17

Natarajan P, DeLucia G, Springel V (2007) Mon Not R Astron Soc 376:180

Natarajan P, Kneib J-P, Smail I, Treu T, Ellis RS, Moran S, Limousin M, Czoske O (2009) Astrophys J 693:970

Navarro J, Frenk C, White S (1997) Astrophys J 490:493

Navarro J et al (2010) Mon Not R Astron Soc 402:21

Neri R et al (2003) Astrophys J Lett 597:113

Newman A, Treu T, Ellis R, Sand D (2011) Astrophys J Lett 728:39

Oguri M, Blandford RD (2009) Mon Not R Astron Soc 392:930

Oguri M et al (2009) Astrophys J 699:1038

Oguri M, Takada M, Okabe N, Smith G (2010) Mon Not R Astron Soc 405:2215

Okabe N, Zhang Y, Finoguenov A, Takada M, Smith G, Umetsu K, Futamase T (2010) Astrophys J 721:875

Okura Y, Umetsu K, Futamase T (2008) Astrophys J 680:1

Ono T, Masai K, Sasaki S (1999) Publ Astron Soc Jpn 51:91

Orban de Xivry G, Marshall P (2009) Mon Not R Astron Soc 399:2

Ota N, Mitsuda K, Fukazawa Y (1998) Astrophys J 495:170

Ota N, Pointecouteau E, Hattori M, Mitsuda K (2004) Astrophys J 601:120

Paczynśki B (1987) Nature 325:572

Paczynśki B, Gorski K (1981) Astrophys J 248:L101

Paraficz D et al (2011) in preparation

Pelló R, Sanahuja B, Le Borgne J, Soucail G, Mellier Y (1991) Astrophys J 366:405

Pelló R et al (1999) Astron Astrophys 346:359

Pelló R et al (2001) Astrophys Space Sci Suppl 277:547

Peng E-H, et al (2009) Astrophys J 701:1283. doi:10.1088/0004-637X/701/2/1283

Perlmutter S et al (1999) Astrophys J 517:565

Pettini M, Steidel CS, Adelberger K, Dickinson M, Giavalisco M (2000) Astrophys J 528:96

Pettini M, Rix S, Steidel C, Hunt M, Shapley A, Adelberger K (2002) Astrophys Space Sci 281:461

Pierre M et al (2007) Mon Not R Astron Soc 382:278

Pires S, Starck J-L, Amara A, Teyssier R, Refregier A, Fadili J (2009) Mon Not R Astron Soc 395:1265

Plagge T et al (2010) Astrophys J 716:1118

Postman M et al (2011) arXiv:1106.3328

Pyne T, Birkinshaw M (1996) Astrophys J 458:46

Refregier A (2003) Mon Not R Astron Soc 338:35

Refregier A, Bacon D (2003) Mon Not R Astron Soc 338:48

Refsdal S (1964a) Mon Not R Astron Soc 128:295

Refsdal S (1964b) Mon Not R Astron Soc 128:307

Richard J, Pello R, Scharer D, Le Borgne J-F, Kneib J-P (2006) Astron Astrophys 456:861

Richard J, et al (2008) Astrophys J 685:705

Richard J, Pei L, Limousin M, Jullo E, Kneib J-P (2009) Astron Astrophys 498:37

Richard J et al (2010) Mon Not R Astron Soc 404:325

Richard J et al (2011) Mon Not R Astron Soc 414:L31

Riemer-Sorensen S, Paraficz D, Ferreira DDM, Pedersen K, Limousin M, Dahle H (2009) Astrophys J 693:1570

Riess A et al (1998) Astron J 116:1009

Rix HW et al (1997) Astrophys J 448:702

Rhodes J et al (2000) Astrophys J 536:79

Romano A et al (2010) Astron Astrophys 514:88

Romer K (2008) In: Conf proc “The X-ray Universe 2008”, Granada, Spain, p 200

Rosati P, Stanford A, Lidman C, Mainieri V, Eisenhardt P (2001) In: Cristiani S, Renzini A, Williams RE (eds) Conf proc "Deep Fields” ESO workshop, Garching, Germany, p 179

Rozo E et al (2010) Astrophys J 708:645 
Rykoff E et al (2008) Astrophys J 675:1106

Saha P, Williams LLR (1997) Mon Not R Astron Soc 292:148

Salvaterra R, Ferrara A, Dayal P (2011) Mon Not R Astron Soc 414:847

Sand D et al (2002) Astrophys J 574:L129

Sand DJ, Treu T, Smith GP, Ellis RS (2004) Astrophys J 604:88

Sand D, Treu T, Ellis R, Smith G (2005) Astrophys J 627:32

Sand D, Treu T, Ellis RS, Smith GP, Kneib J-P (2008) Astrophys J 674:711

Sandage A (1976) Astrophys J 205:6

Santos M, Ellis R, Kneib J-P, Richard J, Kuijken K (2004) Astrophys J 606:683

Schaerer D et al (2007) Astron Astrophys 476:97

Scharf CA, Jones LR, Ebeling H, Perlman M, Malkan M, Wegner G (1997) Astrophys J 477:79

Schneider P (1984) Astron Astrophys 140:119

Schneider P (1995) Mon Not R Astron Soc 302:639

Schneider P, Bartelmann M (1997) Mon Not R Astron Soc 286:696

Schneider P, Ehlers J, Falco E (1992) Gravitational lenses. Springer, Berlin

Schneider P et al (2000) Astron Astrophys 353:41

Seitz C, Schneider P (1995) Astron Astrophys 297:287

Seitz S, Schneider P (1996) Astron Astrophys 305:383

Seitz S, Saglia R, Bender R, Hopp U, Belloni P, Ziegler B (1998) Mon Not R Astron Soc 298:945

Seljak U et al (2005) Phys Rev D 71:103515

Semboloni E et al (2006) Astron Astrophys 452:51

Sereno M (2002) Astron Astrophys 393:757

Sereno M, Longo G (2004) Mon Not R Astron Soc 354:1255

Serlemistos PJ, Smith BW, Boldt EA, Holt SS, Swank JH (1977) Astrophys J 211:L63

Shan H-Y, Kneib J-P, Tao C et al (2011) Astrophys J (submitted). arXiv:1108.1981

Sharon K, Gal-Yam A, Maoz D et al (2010) Astrophys J 718:876

Sheldon ES et al (2001) Astrophys J 554:881

Sheth K, Blain A, Kneib J-P, Frayer D, van der Werf P, Knudsen K (2004) Astrophys J Lett 614:5

Smail I, Dickinson M (1995) Astrophys J Lett 455:L99

Smail I, Couch WJ, Ellis RS, Sharples RM (1995) Astrophys J 440:501

Smail I, Ivison RJ, Blain AW (1997) Astrophys J Lett 490:L5

Smail I et al (1998) Astrophys J 507:L21

Smail I, Ivison R, Blain A, Kneib J-P (2002) Mon Not R Astron Soc 331:495

Smail I et al (2007) Astrophys J Lett 654:33

Smith GP, Kneib J-P, Ebeling H, Czoske O, Smail I (2001) Astrophys J 552:493

Smith GP et al (2002a) Mon Not R Astron Soc 330:1

Smith GP, Smail I, Kneib J-P, Davis CJ, Takamiya M, Ebeling H, Czoske O (2002b) Mon Not R Astron Soc 333:L16

Smith GP, Edge AC, Eke VR, Nichol RC, Smail I, Kneib J-P (2003) Astrophys J Lett 590:L79

Smith GP, Kneib J-P, Smail I, Mazzotta P, Ebeling H, Czoske O (2005) Mon Not R Astron Soc 359:417

Smith GP et al (2009) Astrophys J 707:L163

Smith GP et al (2010) Mon Not R Astron Soc 409:169

Soucail G et al (1987) Astron Astrophys 172:L14

Soucail G, Mellier Y, Fort B, Mathez G, Cailloux M (1988) Astron Astrophys 199:13

Soucail G, Kneib J-P, Bézecourt J, Metcalfe L, Altieri B, Le Borgne J-F(1999) Astron Astrophys 343:L70

Soucail G, Kneib J-P, Golse G (2004) Astron Astrophys 417:L33

Spergel DN et al (2003) Astrophys J Suppl Ser 148:175

Springel V et al (2005) Nature 435:629

Stanishev V et al (2009) Astron Astrophys 507:61

Starck J-L, Piers S, Refregier A (2006) Astron Astrophys 451:1139

Stark D, Ellis R, Richard J, Kneib J-P, Smith G, Santos M (2007) Astrophys J 663:10

Steidel C, Giavalisco M, Dickinson M, Adelberger K (1996) Astron J 112:352

Sullivan M, Ellis R, Nugent P, Smail I, Madau P (2000) Mon Not R Astron Soc 319:549

Suyu S, Halkola A (2010) 524:94

Suyu S, Marshall P, Hobson M, Blandford R (2006) Mon Not R Astron Soc 371:983

Swinbank A et al (2007) Mon Not R Astron Soc 376:479

Swinbank AM et al (2010) Nature 464:733

Thanjavur K, Willis J, Crampton D (2011) Astrophys J 706:571 
Thom (1989) Structural stability and morphogenesis: an outline of a general theory of models. Addison-

Wesley, Reading. ISBN 0-201-09419-3

Thompson RI et al (1999) Astron J 117:17

Treu T et al (2002) ASPC 268:277

Treu T et al (2003) Astrophys J 591:53

Tu H, Limousin M, Fort B, Shu CG, Sygnet JF, Jullo E, Kneib J-P, Richard J (2008) Mon Not R Astron Soc 386:1169

Tyson JA, Wenk RA, Valdes F (1990) Astrophys J Lett 349:L1

Umetsu K, Broadhurst T, Zitrin A, Medezinski E, Hsu L-Y (2011) Astrophys J 729:127

van Albada T, Bahcall JN, Begeman K, Sancisi R (1985) Astrophys J 295:305

van Waerbeke L et al (2000) Astron Astrophys 358:30

Vanderlinde K et al (2010) Astrophys J 722:1180

Vikhlinin A et al (2009) Astrophys J 692:1060

Walsh D, Carswell RF, Weymann RJ (1979) Nature 279:381

Wambsganss J, Bode P, Ostriker J (2004) Astrophys J Lett 606:93

Wang J, White SDM (2009) Mon Not R Astron Soc 396:709

Weinberg NN (1992) Mon Not R Astron Soc 254:315

White SDM et al (2005) Astron Astrophys 444:365

Williams LLR, Navarro JF, Bartelmann M (1999) Astrophys J 527:535

Willis J, Courbin F, Kneib J-P, Minniti D (2008) Mon Not R Astron Soc 384:1039

Wilson G, Cole S, Frenk CS (1996) Mon Not R Astron Soc 280:199

Wittman D, Tyson JA, Kirkman D, Dell'Antonio I, Bernstein G (2000) Nature 405:143

Wittman D et al (2001) Astrophys J 557:L89

Wittman D, Margoniner VE, Tyson JA, Cohen JG, Becker AC, Dell'Antonio IP (2003) Astrophys J 597:218

Wright C, Brainerd T (2000) Astrophys J 534:34

Wyithe S, Oh P, Pindor B (2011) Mon Not R Astron Soc 414:209

Yee H, Ellingson E, Bechtold J, Carlberg R, Cuillandre J-C (1996) Astron J 111:1783

Young P, Gunn JE, Oke JB, Westphal JA, Kristian J (1980) Astrophys J 241:507

Zappacosta L, Buote DA, Gastaldello F, Humphrey PJ, Bullock J, Brighenti F, Mathews W (2006) Astrophys J 650:777

Zaritsky D, Gonzalez AH (2003) Astrophys J 584:691

Zeeman (1977) Catastrophe theory-selected papers 1972-1977. Addison-Wesley, Reading

Zhang Y, Finoguenov A, Bohringer H, Kneib J-P, Smith G, Czoske O, Soucail G (2007) Astron Astrophys 467:437

Zhang Y et al (2010) Astrophys J 711:1033. doi:10.1088/0004-637X/711/2/1033

Zitrin A et al (2010) Mon Not R Astron Soc 408:1916

Zitrin A, Broadhurst T, Barkana R, Rephaeli Y, Benítez N (2011a) Mon Not R Astron Soc 410:1939

Zitrin A, Rosati P, Nonino M et al (2011b). arXiv:1107.2649

Zitrin A, Broadhurst T, Coe D et al (2011c). arXiv:1103.5618

Zwicky F (1933) Helv Phys Acta 6:110

Zwicky F (1937) Astrophys J 86:217 